University of Zurich

Department of Economics

Working Paper Series

ISSN 1664-7041 (print)

ISSN 1664-705X (online)

Working Paper No. 221

\title{
Growth and Welfare Effects of Intellectual Property Rights when Consumers Differ in Income
}

Christian Kiedaisch

Revised version, July 2017 


\title{
Growth and Welfare Effects of Intellectual Property Rights when Consumers Differ in Income*
}

\author{
Christian Kiedaisch ${ }^{\dagger}$
}

July 14, 2017

\begin{abstract}
This paper analyzes how changing the expected length of intellectual property (IP) protection affects economic growth and the welfare of rich and poor consumers. The analysis is based on a product-variety model with non-homothetic preferences and endogenous markups in which, in accordance with empirical evidence, rich households consume a larger variety of goods than poorer ones. The effects of IP protection on growth can be either positive or negative, depending on the distribution of income and wealth. Given that increasing the length of IP protection increases growth, poorer households prefer a shorter length of protection than richer ones.
\end{abstract} (JEL O34, O31, L16, D30, O15)

Keywords: intellectual property rights, income distribution, endogenous growth, nonhomothetic preferences

\section{Introduction}

With income and wealth inequality on the rise in many developed countries (see for example Piketty, 2014), distributional concerns are taking center stage in many policy debates. When it comes to changes in intellectual property right (IPR) policies like those included in the recently negotiated but not ratified Trans-Pacific Partnership agreement (TPP), it is therefore important to understand what their distributional consequences are and whether their effect on innovation and growth depends on the extent of inequality.

${ }^{*}$ I gratefully acknowledge financial support by the Swiss National Science Foundation. I thank Gilles Saint - Paul, Franck Portier, André Grimaud, Vincenzo Denicolò, Reto Föllmi, Josef Zweimüller, Josef Falkinger, Fabrizio Zilibotti, Kiminori Matsuyama, Iain Cockburn, Manuel Amador, Stefan Legge and Holger Strulik for helpful discussions and seminar/ conference participants in Toulouse, Ascona, Aix-enProvence, Vigo, Barcelona, Heidelberg, Boston and Zürich for helpful comments.

${ }^{\dagger}$ Contact: University of Zurich, Department of Economics, Schönberggasse 1, CH-8001 Zürich; Phone: +41 4463437 26; e-mail: christian.kiedaisch@econ.uzh.ch. 
While strengthening intellectual property (IP) protection can have direct distributive effects by raising the relative wages of workers performing $R \& D$ intensive tasks, this paper studies interactions between inequality, IP protection and the demand for new goods. In standard product-variety growth models (e.g. Romer, 1990, or Grossman and Helpman, 1992), the assumption of homothetic preferences (that are identical across households) implies that the demand for individual goods and the incentives to innovate do not depend on the distribution of income. The implication of these models that rich households consume the same variety of goods as poorer ones and just proportionally more of each good, is, however, at odds with empirical evidence: Jackson (1984) and Falkinger and Zweimüller (1996) find that the variety of goods consumed increases in household income. Based on data from the US consumer expenditure survey (CEX), Figure 1 shows that there is also a positive association between a more narrowly defined variety of "innovative" goods purchased by a household and household expenditures.

Figure 1:

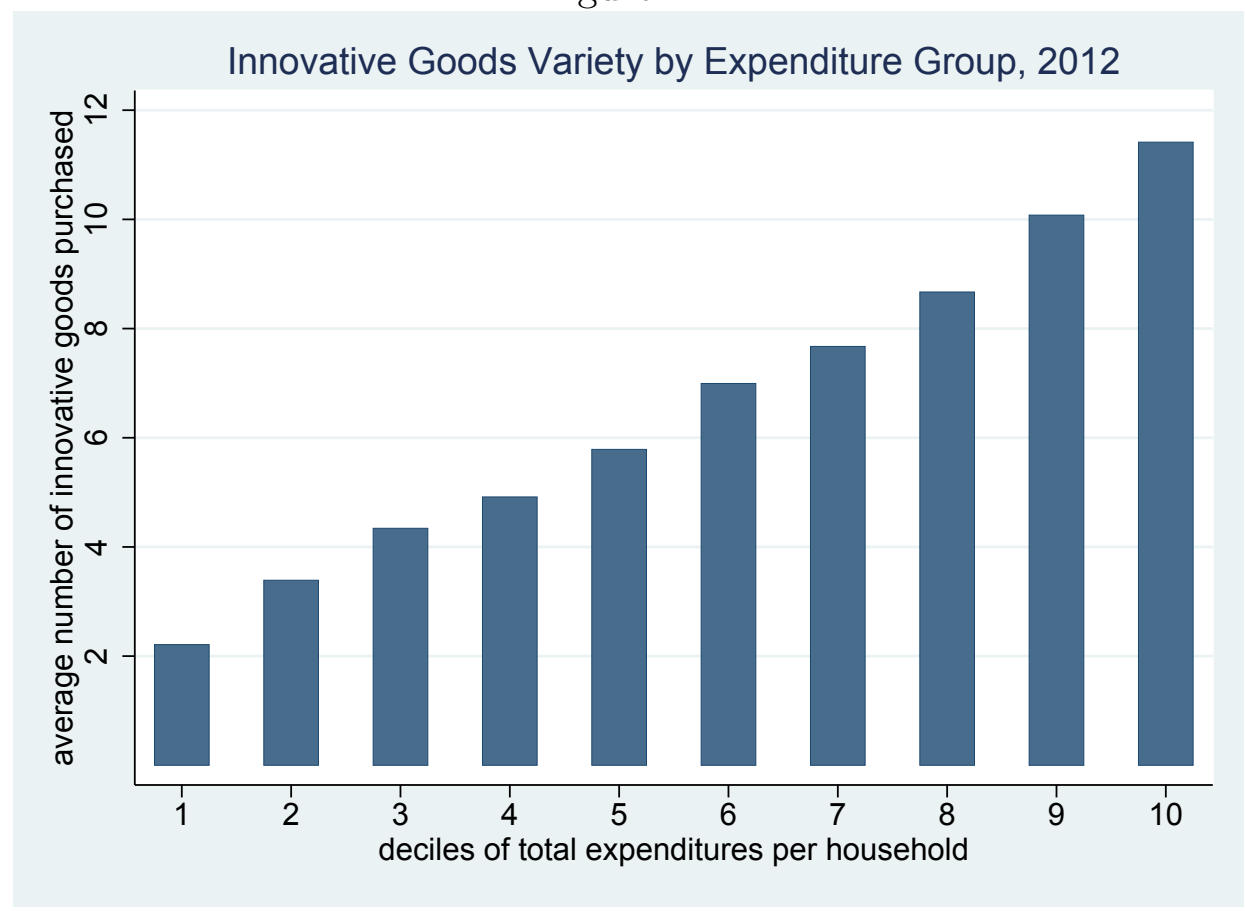

Notes: Out of the over 600 goods from the CEX (INTR), 61 were classified as innovative, among them goods like computer software, video game hardware, portable memory, televisions, photographic equipment and new cars. A complete list of the selected goods is provided in Appendix B1. The number of innovative goods is defined as the number of these selected goods of which a household has purchased at least one unit in 2012. Population weights are those representative for the US population. I thank Liliya Khabibulina for providing this graph.

As rich and poor households differ considerably with respect to their consumption pattern $^{1}$, it is plausible that the demand for individual "innovative" goods depends on

\footnotetext{
${ }^{1}$ The differences in consumption pattern might be even larger when consumers in rich and poor countries are compared.
} 
the distribution of income. If firms that invent new goods rely on IPRs in order to protect their innovations, their profits and incentives to innovate should therefore depend on both the extent of IP protection and the degree of inequality across consumers. Moreover, it is likely that an increase in IP protection which leads to increased markups for some goods affects the consumption of rich and poor households in different ways.

This paper analyzes how varying the expected length of IP protection affects growth and the welfare of rich and poor consumers in a general equilibrium product-variety model with non-homothetic preferences that are identical across households. The analysis is based on Föllmi and Zweimüller (2006), who analyze the effects of inequality on growth, assuming infinite IP protection. In order to introduce non-homotheticity in a simple and tractable way, it is assumed that goods are consumed in discrete amounts and that households are saturated after consuming one unit of a good ${ }^{2}$. This implies that, in line with the empirical evidence, rich households consume a larger variety of goods than poorer ones. Differences in labor productivities are exogenously given, and there is free entry into R\&D. Firms with IP protection engage in monopoly pricing, while goods are sold at marginal cost once IP protection has expired. IP protection is assumed to expire stochastically and, unlike in the previous literature, the general case is considered in which IP policy can have a differential impact on the expiration rates of newly and previously granted IPRs.

Analytical tractability is maintained by parametrizing the distribution of wealth in a special way. This new "modelling trick" allows to derive all results taking transitional dynamics into account. It is important to account for those dynamics as changes in the strength of IP protection lead to changes in the distribution of wealth, so that their effects cannot be understood by simply comparing steady states, holding the extent of inequality constant. Föllmi and Zweimüller (2006) instead only consider the special case in which initial wealth is distributed in the same way as labor endowments, and in which an exogenous change in the distribution of labor endowments is always accompanied by a corresponding exogenous change in the distribution of wealth.

There are two channels through which the effects that IP protection has on growth interact with the level of inequality. On the one hand, the extent to which markups change when the length of IP protection changes depends on the differences in expenditures across households. On the other hand, changes in the length of IP protection can lead to changes

In the CEX data, there is also a positive association between the variety of the remaining "noninnovative" goods and household expenditures, but no clearcut relation between household expenditures and the ratio between the variety of innovative and non-innovative goods that a household purchases. The basic model does not make any predictions about this ratio as it considers the case where all goods are innovative and only distinguishes between goods that are protected by IPRs and others that are not (a characteristic on which the CEX provides no information). In footnote 45 and in Section 5 extensions are discussed in which there are also non-innovative goods and in which rich households consume a larger variety of those goods than poor households.

${ }^{2} \mathrm{An}$ extension with divisible outside goods is discussed in footnote 45. 
in the differences in expenditures (which imply changes in markups) when they affect the value of initial wealth that is held in the form of non-expired IPRs and when this wealth is unequally distributed. If the length of IP protection is uniformly increased for both new and previously granted IPRs, the value of initial wealth remains unchanged (upon impact), implying that the second channel ("valuation effects") is shut down ${ }^{3}$. Then, a (uniform) increase in the length of IP protection reduces the fraction of goods that are competitively supplied and at the same time reduces markups on the increased fraction of monopolistically supplied goods as an increased number of IPR owners compete for a given "total demand".

When both rich and poor households consume IP protected goods ("heterogeneous demand"), a (uniform) increase in the length of IP protection reduces poor households' consumption and increases the rate of growth. When there is no inequality among households who can afford to consume IP protected goods ("uniform demand"), a (uniform) increase in the length of IP protection, however, does not affect growth as it is exactly offset by a reduction in markups and per period profits, leaving the incentives to innovate unchanged. The first main result of the paper is therefore that a (uniform) increase in the length of IP protection is more likely to increase the rate of growth when there is inequality among households consuming IP protected goods ${ }^{4}$.

In the case of heterogenous demand, an increase in the differences in expenditures across households leads to an increase in markups and - as in Föllmi and Zweimüller (2006) - increases the rate of growth. Due to this mechanism, new feedback effects can emerge when the length of IP protection is changed in a non-uniform way, as this implies a change in the value of initial wealth: If the length of IP protection is unexpectedly reduced for future innovations but not for previously issued IPRs, the value of the latter increases due to increasing markups. If wealth is unequally distributed, this leads to an increase in the differences in expenditures across households, which again implies a further increase of markups. In the case where the distribution of wealth is sufficiently unequal, the latter effect can be so strong that a reduction in the length of IP protection can actually increase the rate of growth. All these results differ from those obtained in traditional models with (identical) homothetic preferences, in which markups are constant and in which neither the rate of growth nor the effects of IP protection on growth depend on the distribution of income.

Given that an increase in the length of IP protection increases growth, it reduces the variety of goods consumed by poorer households more (in absolute terms) than that

\footnotetext{
${ }^{3}$ While changes in IP protection do not affect the absolute (normalized) differences in wealth and expenditure levels across households in this case, they do affect the relative levels and therefore wealth inequality in the long run.

${ }^{4}$ It should be noted that the relevant level of inequality is the one among households who are rich enough to consume IP protected goods and not the total level of inequality in the economy, which might actually be larger in the case of "uniform" than in the case of "heterogenous" demand.
} 
consumed by richer households, even though the latter consume a larger variety of IP protected goods (when demand is heterogenous). The intuition for this surprising result is the following: while all households consume all of the goods on which IP protection has expired (as those are the cheapest ones) and are harmed in a similar way when there are fewer of these goods, richer households benefit more from the decline in markups of IP protected goods, as they consume a larger variety of these goods. As poorer households benefit similarly to richer ones when the rate of growth increases due to an increase in the length of IP protection ${ }^{5}$ but suffer from a larger reduction in current consumption, they prefer a shorter duration of IP protection in this case than richer households do. This is the second main result of the paper. Even if IP policies do not affect inequality through supply side forces or valuation effects, they can therefore lead to conflicts of interest when rich and poor households differ with respect to their consumption pattern. Agreeing on a uniform (international) length of IP protection might therefore be a difficult task, as poor households or countries might need to be compensated appropriately in order to support long IP protection ${ }^{6}$.

A further result of the paper is that an increase in the discount rate can lead to an increase in the rate of growth when wealth inequality is sufficiently large. The reason for this is that the former implies an increased propensity to consume out of interest income and that it can therefore increase the inequality in consumption expenditures. As the latter implies larger markups and profits, the value of an innovation can actually increase even though future profits are discounted more heavily. Such a "paradox of thrift" does not arise in standard growth models ${ }^{7}$.

In an extension where firms' price setting power is reduced since households can also consume traditional, non-innovative goods, it is shown that inequality no longer needs encourage growth and that it is more likely to discourage growth, the weaker IP protection is. This is in line with preliminary empirical evidence provided by Dorn and Kiedaisch (2017) who show that patent flows towards a country are more likely positively (and less likely negatively) related to the level of inequality in this country, the larger the strength of patent protection in this country is ${ }^{8}$. This finding is, moreover, in line with the first

\footnotetext{
${ }^{5}$ All households have the same discount rate and there are knowledge spillovers due to which an increase in the number of invented goods increases labor productivities and wages of all households in a proportional way.

${ }^{6}$ In the basic setup of the model, there is no efficient length of IP protection on which all households agree when transfer payments are permitted. The involved distributional conflict can be substantial: in the case of two income groups, poor households actually bear the whole costs in terms of reduced current consumption when the length of IP protection is increased while the consumption of rich households remains unchanged.

${ }^{7}$ While this paradox can even occur in the case of full IP protection, it has not been derived by Föllmi and Zweimüller (2006) who only analyze the case in which the distribution of wealth coincides with the distribution of labor income.

${ }^{8} \mathrm{~A}$ patent flow occurs when a patent with priority filing (first patent application) in one country also gets protected in another country. Dorn and Kiedaisch (2017) argue that patent flows depend in the same qualitative way on inequality and the strength of IP protection as innovation would in a closed
} 
main result of the paper that patents are more likely to increase innovation when there is inequality among households consuming IP protected goods.

In a further extension where markups can be reduced through a reduction in the breadth of IP protection, it is shown that, holding the rate of growth constant, rich households prefer long and narrow IP protection, while poor households prefer short and broad protection.

The paper is structured as follows: In Section 2, the related literature is discussed. Section 3 describes the model setup and Section 4 analyzes the equilibrium. The main part of the analysis focuses on the case of two income groups, with Sections 4.2 and 4.3 discussing the case where demand for IP protected goods is heterogeneous and Section 4.4 the case in which demand is uniform. Section 4.5 studies the welfare effects of IPRs. Section 4.6 extends the analysis to the case where there are many income groups and applies it to an international context. In Section 5, the extension of a limited price setting power is discussed. Section 6 concludes. Proofs are collected in online Appendix A and extensions and supplementary material in online Appendix B.

\section{Related literature}

As this paper builds on Föllmi and Zweimüller (2006), the main differences have already been addressed in the introduction. Two more differences should be mentioned: while Föllmi and Zweimüller (2006) study the case of hierarchical intra-temporal preferences and assume that intertemporal utility is of the CES type, the present paper focuses on the special case in which goods are symmetric and in which intertemporal preferences are logarithmic. This is done in order to keep the analysis simple and tractable. An extension with hierarchical preferences in which innovation is endogenously targeted towards relatively more luxurious goods and in which non-IP protected goods serve relatively more basic needs is analyzed in Appendix B5.

Building on and correcting the analysis of Kwan and Lai (2003), Cysne and Turchick (2012) study the optimal stochastic IP expiration rate in a lab-equipment product variety model based on Rivera-Batiz and Romer (1991). Taking transitional dynamics into account $^{9}$, they find that growth increases when the (expected) length of IP protection is increased ${ }^{10}$. Unlike the present paper, they, however, do not consider the case where the

economy setting. They measure inequality using Gini coefficients and top income shares and the strength of IP protection using the Ginarte-Park index.

${ }^{9}$ If patents are instead assumed to be of finite duration, it becomes very difficult to solve for the transitional dynamics within such models if time is continuous (see Judd (1985) and Cai and Nitta (2012); Deneckere and Judd (1992) and Matsuyama (1999) study models in which time is discrete and in which patents last for one period).

${ }^{10} \mathrm{In}$ standard product variety models, increasing the (expected) length of IP protection always increases steady state growth (see for example Barro and Sala-i-Martin, 2004, Chapter 6.2). Exceptions are Furukawa (2007) and Michel and Nyssen (1998) in which growth can be maximal under finite patent 
IP expiration rate can differ for newly and previously granted IPRs. Cysne and Turchick (2012) find that full IP protection is optimal if preferences over final goods are logarithmic, but not necessarily if preferences are of a more general CES type. In this setup, markups are determined by the constant and exogenously given elasticity of substitution between goods and, unlike in the present paper, do not depend on the length of IP protection or the distribution of income ${ }^{11}$.

Chu (2010) studies a quality ladder model with homothetic preferences in which wealth is unequally and labor incomes are equally distributed. He finds that a (uniform) increase in patent breadth (modeled as an increase in markups) increases the rate of growth, $g$, and the rate of interest, $r$, and that the latter leads to an increase in income inequality. Furthermore, he shows that an increase in patent breadth increases (decreases) consumption inequality if the elasticity of intertemporal substitution, $\varepsilon$, is smaller (larger) than one. The reason for this is that the increases in $g$ and $r$ lead to an increase (decrease) in the propensity to consume out of wealth, $r-g$, if $\varepsilon<1(>1)$. The present paper instead focuses on the case where $\varepsilon=1$ (logarithmic intertemporal preferences), in which changes in $r$ and $g$ do not change the inequality in household expenditures through this channel. Using the setting of Chu (2010) but assuming that R\&D uses final goods instead of labor as inputs, Chu and Cozzi (2016) show that a uniform increase in patent breadth can not only increase inequality by increasing the interest rate, but also by lowering real wages while at the same time leaving the value of initial wealth unchanged. In the present paper, R\&D is undertaken by labor ${ }^{12}$, so that this mechanism is not at work.

In Chu (2010), Chu and Cozzi (2016) and Cysne and Turchick (2012), the assumption of homothetic preferences/technologies implies that (unlike in the present model) households with different income levels but equal ratios of initial wealth to labor income face the same trade-off between growth and current consumption and would therefore prefer the same level of IP protection ${ }^{13}$.

Saint - Paul (2004) studies a two-period model in which only workers with a sufficiently high level of skills decide to become R\&D workers and in which an increase in IP protection increases the skill premium ${ }^{14}$. While a reduction in IP protection might benefit

protection as the former assumes that patent protection reduces learning by doing in the final goods sector and the latter that it prevents other R\&D firms from accessing the patented knowledge.

${ }^{11}$ Föllmi and Zweimüller (2002) introduce hierarchical preferences into a product-variety growth model with a representative consumer. They find that markups for patent protected goods rise over time and that this implies that utility along a balanced growth path is maximal for a finite patent length.

${ }^{12}$ This is a natural specification in the case of non-homothetic preferences in which there is no representative final good (bundle) that could be used as an R\&D input.

${ }^{13}$ This is, however, not shown by the authors. In the setup of Chu (2010), it can, moreover, be shown that in the case where $\varepsilon=1$, all households would prefer the same patent breadth, independent of their wealth to labor income ratio.

${ }^{14}$ Spinesi (2011) and Bernal Uribe (2012) derive similar results in growth models. In Cozzi and Galli (2014) changes in IP legislation that increase the blocking power of basic research relative to applied follow-on research can either increase or decrease growth and the skill premium. In a model with directed technological change, Pan, Zou and Li (2015) find that it is optimal to grant broader patent breadth to 
poor unskilled workers (by reducing prices) and harm rich skilled workers (by reducing their wages), Saint-Paul (2004) argues that a social planner would always prefer to fully enforce IPRs and to address distributional concerns via redistributive taxation. While Saint - Paul (2004) derives the main results assuming homothetic preferences, he also shows the following: if a reduction in IP protection leads to a marginal reduction in the variety of goods that are invented and also in the fraction of monopolistically supplied goods, then poor households are more likely to benefit from it than richer ones if the elasticity of utility with respect to consumption increases in the level of consumption ${ }^{15}$. This result is, however, derived under the assumption that incomes and markups are exogenously given.

In Saint-Paul (2006) the utility derived from the consumption of any single good is bounded from above, implying that, unlike in the case of CES preferences, rich households value an increase in product variety (through innovation) more than poorer ones. While Saint - Paul (2006) does not study the role of intellectual property rights, he shows (in a working paper version, 2002) that a social planner who puts equal weight on each consumer prefers to allocate fewer resources to $R \& D$ than are actually allocated in equilibrium if patents are infinitely lived.

As all of the above-mentioned papers except for Föllmi and Zweimüller (2006) only analyze cases in which each household consumes positive quantities of each good, they, unlike the present paper, are at odds with the empirical evidence indicating that the variety of goods purchased increases in household income.

Hatipoglu (2012) analyzes the effect of inequality on demand-induced innovations when patents are of finite length. The theoretical part of his analysis builds on Zweimüller (2000), who studies a growth model with hierarchical preferences in which markups are exogenously given. Hatipoglu (2012) finds that redistributing wealth from rich to poor households can increase demand for patent protected goods and growth by allowing poor households to overcome a critical income threshold below which they cannot afford to purchase patent protected goods. This effect disappears in the present model in which markups are endogenous (see footnote 39 ).

In Kiedaisch (2009), I study a two-period product-variety model with strictly hierarchical preferences and sector-specific cost-saving innovations. While an increase in IP protection always increases innovation when profit and labor incomes are equally dis-

skill-complementary innovations and that this encourages skill-biased technological change and leads to an increase in wage inequality.

In empirical analyses based on cross country panel data, Adams (2008) and Saini and Mehra (2014) find that strengthening intellectual property protection has increased income inequality in developing countries and Saini and Mehra (2014) find that it has reduced income inequality in developed countries. Using US cross-state panel data, Aghion et al. (2016) find a positive effect of innovation on top income inequality.

${ }^{15}$ That means if $c \frac{u^{\prime}(c)}{u(c)}$ increases in $c$, where $u(c)$ denotes the utility derived from a single good (total utility is additive). 
tributed, there are two opposing effects when all profit income accrues to a small minority of rich households: then, stronger IP protection encourages innovation in basic need sectors but - by reducing the purchasing power of mass consumers - reduces demand and the incentives to innovate in sectors that produce more luxurious goods, implying that "overall innovation" might be reduced. Unlike in the present paper, markups are constant in Kiedaisch (2009) and, due to the lack of free entry into R\&D, an increase in IP protection increases the rents of firm owners.

Within an international context, several papers have analyzed the effects of having different levels of IP protection in different countries that interact in a global economy ${ }^{16}$. Contrary to this literature, the present paper focuses on the question whether differences in the structure of demand can lead to disagreement about the optimal uniform global strength of IP protection.

Furthermore, the paper relates to an extensive literature about the relationship between inequality and growth and specifically to a few papers in which inequality affects innovation and growth through the channel of demand ${ }^{17}$.

\section{The model setup}

\subsection{Preferences and technology}

There is a continuum of potentially producible differentiated goods indexed by $j \in[0, \infty)$. In a given period $t$, only one or zero units of any of these goods can be consumed by a household $i: c_{i}(j, t) \in\{0,1\}$.

Households are infinitely lived and intertemporal utility is given by:

$$
U_{i}(\tau)=\int_{t=\tau}^{\infty} \ln \left(\int_{j=0}^{\infty} c_{i}(j, t) d j\right) e^{-\rho(t-\tau)} d t
$$

\footnotetext{
${ }^{16}$ Chung and $\mathrm{Lu}(2014)$ analyze the effects of southern IP protection in a two-period North-South model with hierarchical preferences in which innovators can supply goods of different quality levels. There is no within-country inequality in this model and, due to the lack of arbitrage, goods are sold at different prices in the North and the South. Kohler (2012) studies a dynamic North-South trade model with non-homothetic preferences and full IP protection. He finds that preventing arbitrage across countries reduces innovation and harms the rich North while it might benefit the poor South. Chu and Peng (2011) extend the model of Chu (2010) to a two-country context. Further references can be found in a recent literature survey by Saggi (2016).

${ }^{17}$ See Murphy et al. (1989), Falkinger (1994), Zweimüller (2000), Mastuyama (2002), Chou and Talmain (1996), Föllmi and Zweimüller (2006), Föllmi and Zweimüller (2016), Würgler (2010) and Föllmi, Würgler and Zweimüller (2014) for theoretical contributions. In an empirical study, Beerli et al (2014) find that changes in the Chinese income distribution led to considerable changes in market sizes for different durable goods and that productivity increased in sectors in which demand increased. Studying product-level data from the US retail sector (2004 - 2015), Jaravel (2017) finds that increasing inequality implied a faster increase in product variety for rich than for poor households and that this went along with lower inflation rates for richer households.
} 
where $\rho>0$ denotes the rate of time preference. While preferences are homothetic in the intertemporal dimension, the strong assumption of indivisibilities in the consumption of goods (" $0-1$ consumption") is made in order to introduce non-homothetic intra-temporal preferences in a simple and tractable way ${ }^{18}$.

The factors of production are homogenous labor and the measure $N(t)$ of goods that have been invented until point in time $t$ (the "stock of knowledge"). Producing one unit of an invented good requires $\frac{b}{N(t)} \geq 0$ units of labor as an input and attaining an innovation in a sector $j$ is associated with fixed R\&D costs equal to $\frac{F}{N(t)}>0$ units of labor. The assumption that labor productivity in both the production and the R\&D sector increases in this multiplicative way in the stock of knowledge $N(t)$ is made in order to allow for continuous exponential growth ${ }^{19}$.

\subsection{Intellectual property protection and prices}

The labor market is assumed to be competitive and there is free entry into R\&D. An innovator who has invented a good $j$ obtains intellectual property (IP) protection on it, which allows him to exclude others from producing this good. The intellectual property right, however, does not allow appropriating any of the spillovers, which increase the productivity of both firms that produce other goods and of future innovators, implying that there is a research exemption ${ }^{20}$. IP protection is assumed to expire with hazard rate $\gamma$ (that means with probability $\gamma d t$ in time interval $d t$ ), implying that the expected length of protection is equal to $T \equiv \frac{1}{\gamma}$. IPRs are therefore infinitely lived $(T=\infty)$ if $\gamma=0$ and not protected at all $(T=0)$ if $\gamma \rightarrow \infty^{21}$.

After the IPR on a good $j$ has expired, anyone can freely produce this good and it is supplied at marginal cost due to perfect competition ${ }^{22}$. The market clearing wage is

\footnotetext{
${ }^{18}$ Under certain circumstances, the model might also be interpreted as one in which there is a single final consumption good of which different quality levels can be consumed: when a higher quality level can only be produced when a larger variety of inputs $j$ are used in the production process, consuming a final good of higher quality becomes similar to consuming a larger variety of goods (a high quality software might for example consist of a low quality version to which new features are added).

${ }^{19}$ Unlike in standard growth models, the productivity of the production sector needs to increase in $N(t)$ as the assumption of consumption indivisibilities precludes the possibility to consume and produce less of each good when the number of goods increases. Only in the special case of a "digital economy" where $b=0$, these spillovers are not required (see footnote 32 ). If there were no spillovers in the R\&D sector, growth would be linear but the qualitative results would be the same. A simple two-period version of the model without any spillovers is studied in Appendix B8.

${ }^{20}$ As R\&D productivity increases in the stock of knowledge $N(t)$, future innovators benefit from the R\&D undertaken by previous innovators. IP protection could therefore be broadened by granting innovators some blocking power over future inventions which would enable them to extract licensing fees from future innovators. In Appendix B3 it is shown that granting such extended IP protection would reduce the rate of growth along a balanced growth path.

${ }^{21}$ In the following, $T$ will often simply be referred to as the "length of IP protection". In the case of a finite deterministic length of IP protection or infinitely lived IPRs that are only enforced with a certain probability at the time of invention, the qualitative results stay the same as long as balanced growth paths are compared (see Appendix B4).

${ }^{22}$ The analysis focuses on intellectual property rights as the only factor granting a monopoly position.
} 
denoted by $w(t)$. In order to obtain constant prices for the competitively supplied goods, the wage of a productivity-adjusted unit of labor is normalized to one, implying that the wage for one unit of labor is normalized to $w(t)=N(t)$. Due to this normalization, the marginal production costs of a good and therefore the price of goods on which IP protection has expired is given by $p(j, t)=b$. The fixed $\mathrm{R} \& \mathrm{D}$ costs are also constant over time and given by $F$, as R\&D labor is competitively supplied. It is assumed that a firm with IP protection in sector $j$ cannot observe its customers' income and therefore cannot price discriminate between households with different willingness to pay.

Households are not financially constrained and can borrow and lend at the interest rate $r(t)$.

\subsection{Distribution}

The size of the population and the total labor endowment of the economy are normalized to 1 . While all households have the same utility function, it is assumed that there are poor $(P)$ and rich $(R)$ households with population shares $\beta$ and $1-\beta$ (with $0 \leq \beta<1$. The case of many income groups is analyzed in Section 4.6). A poor household's labor endowment is given by $l_{P}=\vartheta(0<\vartheta \leq 1)$ and that of a rich household by $l_{R}=\frac{1-\beta \vartheta}{1-\beta} \geq 1$, as $\beta l_{P}+(1-\beta) l_{R}=1$ must hold. For a given $\vartheta<1, l_{R}$ therefore increases in $\beta$. A household's labor endowment pins down its labor income as a share of the average labor income in the economy. Inequality in labor incomes is said to increase if $\beta$ increases or if $\vartheta$ decreases ${ }^{23}$.

At the initial date $t=\tau$, the economy is endowed with wealth in the form of previously granted non-expired IPRs, the value of which is equal to the expected discounted profit income accruing to their owners. A rich household's initial wealth is denoted by $V_{R}(\tau)$ and that of a poor household by $V_{P}(\tau)$, and it is assumed that $V_{R}(t) \geq V_{P}(t) \geq 0$ holds $^{24}$.

\subsection{Consumption choices}

The intertemporal budget constraint of a household of type $i \in\{R, P\}$ is given by:

$$
\int_{t=\tau}^{\infty} N(t) l_{i} e^{-R(t, \tau)} d t+V_{i}(\tau) \geq \int_{t=\tau}^{\infty}\left(\int_{j=0}^{\infty} p(j, t) c_{i}(j, t) d j\right) e^{-R(t, \tau)} d t
$$

If such a position can be obtained through other factors like trade secrecy, the same analysis applies to these factors as long as there are the same spillovers and as long as the monopoly position can be lost due to imitation with hazard rate $\gamma$.

${ }^{23}$ The Gini coefficient is given by $G=\beta(1-\vartheta)$.

${ }^{24}$ The analysis can be extended to cases where $V_{R}(t)<V_{P}(t)$, where $V_{i}(t)<0$ for one of the groups (debt), or where $\vartheta>1$. As long as the distribution of labor endowments and initial wealth is such that a rich household is overall richer than a poor household and spends more in every period, this does not change the (qualitative) results. 
where $R(t, \tau)=\int_{s=\tau}^{t} r(s) d s$ is the cumulative discount rate between dates $\tau$ and $t$. The left hand side represents the discounted sum of wage income (note that $w(t)=N(t)$ ) plus the value of initial wealth; the right hand side denotes the discounted sum of consumption expenditures $(p(j, t) \rightarrow \infty$ when a good is not yet invented). A household maximizes intertemporal utility (equation 1) subject to this budget constraint. As preferences are additively separable across periods, this maximization problem can be solved by applying two-stage budgeting: the household first maximizes instantaneous utility by maximizing the measure of goods $C_{i}(t)=\int_{j=0}^{\infty} c_{i}(j, t) d j$ that can be purchased with given expenditures $E_{i}(t)=\int_{j=0}^{\infty} p(j, t) c_{i}(j, t) d j$ in a period $t^{25}$ and then optimally allocates expenditures across periods. The first problem is solved by purchasing one unit of all goods the prices of which lie below the household's willingness to pay $z_{i}(t)$ and a non-negative measure of goods with prices equal to $z_{i}(t)^{26}$. Then, a Lagrangian with the variables $C_{i}\left(E_{i}(t)\right)$ and $E_{i}(t)$ can be set up and maximized with respect to $E_{i}(t)$ in order to derive $z_{i}(t)$. The resulting optimal consumption rule is given by:

$$
c_{i}(j, t)=\left\{\begin{array}{cr}
1 \text { if } & p(j, t)<\frac{e^{R(t, \tau)-\rho(t-\tau)}}{\mu_{i} C_{i}(t)} \equiv z_{i}(t) \\
1 \text { or } 0 \text { if } & p(j, t)=z_{i}(t) \\
0 \text { if } & p(j, t)>z_{i}(t)
\end{array}\right.
$$

where $\mu_{i}$ is the Lagrange multiplier and represents the marginal utility of income at the initial date $\tau$. Given that rich households spend more on consumption in a given period than poor households, they also consume a larger variety (measure) of goods, i.e. $C_{R}(t)>C_{P}(t)$. In equilibrium, the intertemporal budget constraints are satisfied with equality (implying that $z_{i}(t)<\infty$ ) and the willingness to pay of a rich household exceeds that of a poor household, so that $z_{R}(t)>z_{P}(t)\left(\text { and } \mu_{R}<\mu_{P}\right)^{27}$.

\subsection{Monopoly pricing}

A firm that has IP protection on good $j$ sets the price $p(j, t)$ in order to maximize profits. As $z_{R}(t)>z_{P}(t)$, market demand for any good $j$ in period $t$ is given by a step function (see Figure 2): for a price higher than the willingness to pay of a rich household $\left(p(j, t)>z_{R}(t)\right)$, there is no demand for the good; for a price equal to or below the willingness to pay of a rich but above that of a poor household $\left(p(j, t) \in\left(z_{P}(t), z_{R}(t)\right]\right)$,

\footnotetext{
${ }^{25}$ As all goods enter symmetrically into the utility function, households only care about the total measure of goods consumed.

${ }^{26}$ There is always a positive measure of such goods in equilibrium, implying that $\frac{\partial C_{i}(t)}{\partial E_{i}(t)}=\frac{1}{z_{i}(t)}$ holds.

${ }^{27}$ In an extension that is discussed in Section $5, z_{R}(t)=z_{P}(t)$ can hold in a particular regime (C3).
} 
demand is given by the population size of the rich, $1-\beta$; and for a price below or equal to the willingness to pay of a poor household $\left(p(j, t) \leq z_{P}(t)\right)$ demand is equal to one (the size of the whole population).

In order to maximize profits, an IP holding firm then either sets $p(j, t)=z_{R}(t)$ and sells only to rich households (point A in Figure 2) or charges $p(j, t)=z_{P}(t)<z_{R}(t)$ and sells to both rich and poor households (point B). In the first case, profits are given by $\pi_{R}(t)=(1-\beta)\left(z_{R}(t)-b\right)$, and by $\pi_{P}(t)=z_{P}(t)-b$ in the second case (note that marginal production costs are equal to $b$ ). The firm therefore charges low prices and sells to the whole population if $\pi_{P}(t)>\pi_{R}(t)$, charges high prices and sells only to rich households if $\pi_{R}(t)>\pi_{P}(t)$, and is indifferent between the two strategies if $\pi_{P}(t)=\pi_{R}(t)$.

\subsection{Price structure and regimes}

The subset of sectors in which IP protection has expired is denoted by $M(t)<N(t)$ and the definition $m(t) \equiv \frac{M(t)}{N(t)}$ is used. While prices are equal to $b$ in these sectors, the prices in the sectors in which IPRs are protected (of which there is a measure $N(t)-M(t)$ ) depend on the distribution of income and other parameters of the model.

Given that rich and poor households spend the amounts $E_{R}(t)$ and $E_{P}(t)\left(<E_{R}(t)\right)$ in period $t$, either $C_{P}(t)<C_{R}(t)<N(t)$ or $C_{P}(t)<C_{R}(t)=N(t)$ holds $^{28}$. The case where $C_{P}(t)<C_{R}(t)<N(t)$ can arise if even rich households do not spend enough to be able to purchase all invented goods, even if they are sold at marginal cost (e.g. if $\left.E_{R}(t)<b N(t)\right)$. As households value all goods equally, competition between firms then implies that no good is sold at a price that exceeds the marginal cost of $b$ in equilibrium. As even IP holding firms do not earn any profits in this case, there are no incentives to undertake costly $\mathrm{R} \& \mathrm{D}$ and there cannot be positive growth. The more interesting case in which IP holding firms earn positive profits arises when $E_{R}(t)>b N(t)$. Then, $C_{P}(t)<C_{R}(t)=N(t)$ holds, meaning that rich households consume one of each of the invented goods while poor households just consume the fraction $c_{P}(t) \equiv \frac{C_{P}(t)}{N(t)}$ of those goods (referred to as their "consumption share"). The following analysis focuses on this case in which two different regimes can emerge:

In Regime $\mathbf{A}, C_{P}(t)>M(t)$ (Condition $\mathbf{A}$ ) so that poor households not only consume goods the IPRs of which have expired but also some more expensive IP protected goods. Part of the IP protected goods are then exclusively sold to rich households at the price $p_{R}(t)=z_{R}(t)$ while others are sold to both rich and poor households at the price $p_{P}(t)=z_{P}(t)(>b)$. As IP holding firms supply symmetric goods, they must be indifferent

\footnotetext{
${ }^{28}$ The case where $C_{P}(t)=C_{R}(t)=N(t)$ cannot be an equilibrium for the following reason: If rich households purchased the same measure of goods as poor households (at the same prices), they would not exhaust their budgets, implying that their willingness to pay for an additional good, $z_{R}(t)$, would be infinitely large. Then, some firms would have an incentive to increase their price and to sell exclusively to rich households, implying that poor households would not purchase one of each of the invented goods anymore.
} 
between both strategies so that $\pi_{R}(t)=(1-\beta)\left(p_{R}(t)-b\right)=\pi_{P}(t)=\left(p_{P}(t)-b\right)$ has to hold. This implies that

$$
p_{P}(t)=z_{P}(t)=\beta b+(1-\beta) p_{R}(t)
$$

Firms that sell to both groups therefore charge a price that is lower than the price $p_{R}(t)=z_{R}(t)$ charged by firms that sell exclusively to rich households.

In Regime $\mathbf{B}, C_{P}(t)<M(t)$ (Condition $\mathrm{A}$ is violated) holds so that poor households only consume goods the IPRs of which have expired. This regime arises if $E_{P}(t)<$ $b M(t)$, i.e. if the spending of a poor household is small relative to the measure $M(t)$ of competitively supplied goods. As poor households' willingness to pay is then equal to marginal cost $\left(z_{P}(t)=b\right)$, IP holding firms face a "uniform" demand from rich households and sell exclusively to them at the price $p_{R}(t)=z_{R}(t)(>b)$.

In the following sections the equilibrium of the dynamic model is derived and the endogenous variables $N(t), M(t), E_{i}(t), C_{i}(t), p_{j}(t), \pi_{j}(t), r(t)$ and $z_{i}(t)$ are derived as functions of the exogenous parameters.

\section{The general equilibrium}

This section studies the general equilibrium of the model. The analysis focuses on Regime A in which demand for IP protected goods is heterogeneous and where even poor households purchase some IP protected goods in equilibrium, so that $C_{P}(t)>M(t)$ (Condition A) holds. Regime B, in which IP holding firms face a uniform demand from rich households is briefly analyzed in Subsection 4.4.

\subsection{The allocation of resources across sectors}

In Regimes $\mathrm{A}$ and $\mathrm{B}$, the demand for production labor $L_{D}$ in period $t$ is given by $L_{D}(t)=$ $\int_{j=0}^{\infty}\left(\frac{b}{N(t)}\right)\left[\beta c_{P}(j, t)+(1-\beta) c_{R}(j, t)\right] d j=b \beta c_{P}(t)+b(1-\beta)$ as $\frac{b}{N(t)}$ units of labor are needed in order to produce one unit of a good and as the population size of poor (rich) households is given by $\beta(1-\beta)$. The simplification arises as $C_{R}(t)=\int_{j=0}^{\infty} c_{R}(j, t) d j=N(t)$ and as poor households only consume a subset $c_{P}(t) \equiv \frac{C_{P}(t)}{N(t)}$ of the existing goods. The demand $L_{R}$ for R\&D workers depends on how much research is undertaken, meaning on $\dot{N}(t)=\frac{\partial N(t)}{\partial t}$. As the invention of a new product requires $F / N(t)$ units of labor, the demand for R\&D workers is given by: $L_{R}(t)=F \frac{N^{\bullet}(t)}{N(t)}=F g(t)$, where $g(t)$ denotes the rate of growth of the stock of knowledge $N(t)$. Equating supply and demand of labor 
in a given period yields $1=L_{D}(t)+L_{R}(t)$. Plugging the corresponding values into this equation and solving for $g(t)$ gives the economy's resource constraint:

$$
g(t)=\frac{1}{F}\left[1-b \beta c_{P}(t)-b(1-\beta)\right]
$$

Given that $b>0$, there is a negative relation between the rate of growth $g(t)$ and the consumption share of poor households, $c_{p}(t)$. The reason for this is that, as rich households always consume one of each of the invented goods in equilibrium, employing more workers in the $\mathrm{R} \& \mathrm{D}$ sector is only possible if fewer workers are used to produce goods for poor households. ${ }^{29}$

\subsection{Balanced growth}

\subsubsection{Value of an innovation and interest rate}

The expected value of an innovation $Z(t)$ is equal to the expected discounted sum of profit income that accrues to an IPR holder. Therefore, $Z(t)=\int_{s=t}^{\infty} \pi(s) e^{-\tilde{R}(s, t)} d s$, with $\tilde{R}(s, t)=\int_{q=t}^{s}(r(q)+\gamma) d q$ denoting the cumulative discount rate between dates $t$ and $s$ that depends on both the interest rates and the hazard rate $\gamma$ at which profits are lost due to expiring IP protection. Deriving $z_{i}(t)=p_{i}(t)=\frac{e^{R(t, \tau)-\rho(t-\tau)}}{C_{i}(t) \mu_{i}}$ with respect to time gives the Euler equation: $\frac{\dot{C}_{i}(t)}{C_{i}(t)}=r(t)-\rho-\frac{\dot{p}_{i}(t)}{p_{i}(t)}$. As $\frac{\dot{p}_{R}(t)}{p_{R}(t)}=\frac{\dot{p}_{P}(t)}{p_{P}(t)}$ according to equation 4, $\frac{\dot{C}_{P}(t)}{C_{P}(t)}=\frac{\dot{C}_{R}(t)}{C_{R}(t)}=\frac{\dot{N}(t)}{N(t)}=g(t)$ has to hold in any equilibrium, implying that as $c_{P}(t)=\frac{C_{P}(t)}{N(t)}$ must be constant. Along a balanced growth path (BGP), not only $g(t)$ and $c_{P}(t)$, but also per period profits $\pi(t)=(1-\beta)\left(p_{R}(t)-b\right)=\left(p_{P}(t)-b\right)$ and the willingness' to pay $z_{i}(t)=p_{i}(t)$ are constant. The Euler equation then implies that:

$$
r(t)=\rho+g(t)
$$

The rate of interest is therefore constant along a BGP and positively related to the rate of growth and to the rate of time preference. Along a BGP, the expected value of an innovation is consequently given by

$$
Z(t)=\frac{\pi}{r+\gamma}=\frac{(1-\beta)\left(p_{R}-b\right)}{\rho+g+\gamma}
$$

Due to free entry into $\mathrm{R} \& \mathrm{D}$, the value of an innovation $Z$ has to be equal to the (wage) costs of innovating, which are given by $F$. Therefore, the following free entry

\footnotetext{
${ }^{29}$ This negative relation between $g(t)$ and $c_{p}(t)$ only disappears in the case of a "digital economy" where marginal production costs are zero $(b=0)$.
} 
condition needs to hold along a BGP with positive growth:

$$
Z=\frac{(1-\beta)\left(p_{R}-b\right)}{\rho+g+\gamma}=F
$$

\subsubsection{Equilibrium price structure}

In the following, the BGP values of $m(t) \equiv \frac{M(t)}{N(t)}$ and $p_{R}$ are derived.

Multiplying the measure $N(t)-M(t)$ of sectors in which IPRs are protected with the hazard rate $\gamma$ with which IP protection expires, the absolute increase in the measure $M(t)$ of sectors in which IP protection has expired is given by $\dot{M(t)}=\gamma(N(t)-M(t))$. Taking into account that $g(t)=\frac{\dot{N}(t)}{N(t)}$, we can derive $\dot{m}(t)=\gamma(1-m(t))-m(t) g(t)$. Along a BGP, $\dot{m}=0$ needs to hold, so that

$$
m=\frac{\gamma}{g+\gamma}
$$

Given that $c_{p}>m$ holds (Condition A; Regime A), the BGP consumption expenditures of a poor household in period $t$ are given by

$$
\int_{j=0}^{\infty} p(j, t) c_{P}(j, t) d j=N(t)\left[m b+p_{P}\left(c_{p}-m\right)\right]
$$

and those of a rich household by

$$
\int_{j=0}^{\infty} p(j, t) c_{R}(j, t) d j=N(t)\left[m b+p_{P}\left(c_{P}-m\right)+p_{R}\left(1-c_{P}\right)\right]
$$

While both rich and poor households consume the fraction $m$ of non-IP protected goods and the fraction $c_{P}-m$ of IP protected goods that are sold at the low price $p_{P}$, only rich households consume the fraction $1-c_{P}$ of IP protected goods that are sold at the high price $p_{R}$. A graphical representation of the equilibrium price and consumption structure is given in Figure 3. The per period labor income of a poor household is given by $w(t) l_{P}=N(t) \vartheta$ and that of a rich household by $w(t) l_{R}=N(t) \frac{1-\beta \vartheta}{1-\beta}$. Using the relation $N(t)=N(\tau) e^{g(t-\tau)}$ and equation (4), the intertemporal budget constraint of a poor household in period $t=\tau$ is therefore given by:

$$
\frac{N(\tau) \vartheta}{r-g}+V_{P}(\tau)=\frac{N(\tau)}{r-g}\left[m b+\left(b \beta+(1-\beta) p_{R}\right)\left(c_{P}-m\right)\right]
$$

For a rich household, the intertemporal budget constraint is given by:

$$
\frac{N(\tau)(1-\beta \vartheta)}{(r-g)(1-\beta)}+V_{R}(\tau)=\frac{N(\tau)}{r-g}\left[m b+\left(b \beta+(1-\beta) p_{R}\right)\left(c_{P}-m\right)+p_{R}\left(1-c_{p}\right)\right]
$$


Multiplying both sides of equations 9 and 10 by $r-g=\rho$ (see equation 6 ), we obtain the consumption expenditures in period $\tau$ on the right hand sides. Expenditures of a poor household in any period $t=\tau$ are therefore equal to $E_{P}(\tau)=N(\tau) \vartheta+\rho V_{P}(\tau)$ and those of a rich household are equal to $E_{R}(\tau)=N(\tau) \frac{1-\beta \vartheta}{1-\beta}+\rho V_{R}(\tau)>E_{P}(\tau)$. Along the BGP, households therefore spend all their labor income $\left(w(t) l_{i}=N(t) l_{i}\right)$ in each period and the fraction $(r-g) V_{i}(t)=\rho V_{i}(t)$ of their interest income $r V_{i}(t)$, implying that expenditures $E_{i}(t)$ and individual wealth $V_{i}(t)$ grow at rate $g$. This implies that $\frac{V_{R}(t)}{V_{P}(t)}=\frac{V_{R}(\tau)}{V_{P}(\tau)}$ holds, i.e. that the relative distribution of wealth stays constant over time along a BGP and that it reflects the initial distribution, independently of the distribution of labor endowments and incomes, i.e. of $\frac{l_{R}}{l_{P}}$.

As there is a measure $N(t)(1-m)$ of sectors in which IP protection has not yet expired and as the value of an IP protected innovation is given by $Z=F$ (see (7)), the total value of initial wealth along a BGP is given by

$$
V(t)=N(t)(1-m) Z=N(t)(1-m) F
$$

When $V(t)$ changes due to a change in $m$, also $V_{R}(t)$ and $V_{P}(t)$ change. As will be proven in Section 4.3 which studies the transitional dynamics, these changes in $V_{i}(t)$ are (unless IP protection is changed in a non-uniform way) of equal absolute size $\mathrm{s}^{30}$. Because of that, the following special parametrization of the wealth distribution is used:

$$
V_{R}(t)=V_{P}(t)+X N(t)
$$

where $X \geq 0$ is a parameter that stays constant along a BGP (but might jump during a transition). Using equation 11 and the relation $V(t)=\beta V_{P}(t)+(1-\beta) V_{R}(t)$, we can derive the individual BGP wealth levels as

$$
\begin{gathered}
V_{R}(t)=(1-m) F N(t)+\beta X N(t) \\
V_{P}(t)=(1-m) F N(t)-(1-\beta) X N(t)
\end{gathered}
$$

This parametrization ensures that $V_{R}(t), V_{P}(t)$ and $N(t)$ all grow at rate $g$ along a BGP when $X$ is constant, implying a constant relative wealth distribution (i.e. that $\frac{V_{R}(t)}{V_{P}(t)}=\frac{V_{R}(\tau)}{V_{P}(\tau)}$ does not change over time). The analysis focuses on the case where $V_{P}(t)>0$ (Condition B) holds. Dividing equation 13 by equation 14 and solving for $X$ gives $X=\frac{(1-m) F\left(\frac{V_{R}}{V_{P}}-1\right)}{\beta+(1-\beta) \frac{V_{R}}{V_{P}}}$. For any fixed BGP value of $m$, any particular wealth distribution $\frac{V_{R}(t)}{V_{P}(t)}$ can therefore be generated by setting $X$ equal to this value (which increases in $\frac{V_{R}(t)}{V_{P}(t)}$ and decreases in $m$ ).

\footnotetext{
${ }^{30}$ The reason for this is that both rich and poor households consume the same absolute measure $N(t) m$ of goods on which IP protection has expired and are therefore affected in a similar way when $m$ changes.
} 
The "modelling trick" of parametrizing the distribution of wealth in this special way makes the following analysis tractable. The reason for this is that a change in $m$ resulting from a change in an exogenous parameter turns out to leave $X \equiv \frac{V_{R}(t)-V_{P}(t)}{N(t)}$ unchanged (unless a non-uniform change in IP protection is considered), while it leads to a change in the relative wealth distribution $\frac{V_{R}(t)}{V_{P}(t)}$.

Plugging equation 12 into equation 10, subtracting equation 9 from equation 10, and solving for $p_{R}$ gives:

$$
p_{R}=\frac{\frac{1-\vartheta}{1-\beta}+\rho X}{1-c_{P}}
$$

$p_{R}$ therefore increases in $c_{P}, \beta, X$ and $\rho$ and decreases in $\vartheta$. The mechanism behind these results is the following: the entire expenditure difference $E_{R}(t)-E_{P}(t)=N(t)\left(l_{R}-l_{P}\right)+$ $\rho\left(V_{R}-V_{P}\right)=N(t)\left(\frac{1-\vartheta}{1-\beta}+\rho X\right)$ is used to purchase the measure $N(t)\left(1-c_{P}\right)$ of IP protected goods that are each sold at price $p_{R}$. For a given expenditure difference, $p_{R}$ must therefore increase in $c_{P}$, as rich households spend all their money on the existing goods. A reduction in $\vartheta$ and an increase in $\beta$ increase the expenditure difference by increasing the difference in labor income $N(t)\left(l_{R}-l_{P}\right)$, and an increase in $X$ or $\rho$ increases it by increasing the difference in consumption out of interest income. For $c_{P}$ given, this increased expenditure difference can only be absorbed if $p_{R}$ increases.

\subsubsection{Properties of the BGP}

Plugging equation 15 into equation 7 and solving for $g$, the free entry condition can be written as:

$$
g=\left(\frac{\frac{1-\vartheta}{1-\beta}+\rho X}{1-c_{P}}-b\right) \frac{1-\beta}{F}-\rho-\gamma
$$

The growth rate $g$ depends positively on $c_{P}, X$ and $\beta$ and negatively on $\vartheta, \gamma$ and $F$. The reason for this is the following: an increase in $c_{P}, X$, and $\beta$ and a decrease in $\vartheta$ increase the value of an innovation by increasing $p_{R}^{31}$ and an increase in $\gamma$ reduces the value of an innovation by shortening the length of IP protection. An increase in $F$, on the other hand increases the costs of innovating. As the value of an innovation decreases if the interest rate $r$ increases due to an increase in the rate of growth (remember that $r=\rho+g$ (equation 6)), the free entry condition, which equates the value of an innovation to the costs of innovating implies a positive relation between $g$ and the value of an innovation and a negative relation between $g$ and $F$. The effect of $\rho$ on $g$ depends on the size of $X$ : For small values of $X$, the free entry condition implies a negative relation between $g$ and $\rho$ which is mainly driven by the fact that an increase in $\rho$ reduces the value of

\footnotetext{
${ }^{31}$ In the case where $\beta$ increases, this effect is weakened by a reduction in the relevant market size, $1-\beta$. However, the value of an innovation still increases as the first effect is stronger than the second one.
} 
an innovation through a discounting effect. For large values of $X$, this discounting effect can, however, be dominated by a positive price effect that results from an increase in the expenditure difference between rich and poor households: As an increase in $\rho$ increases the consumption out of interest income $\rho V_{i}(t)$, it also increases the expenditure difference and therefore $p_{R}$ and this effect is stronger the larger $X$ is.

The free entry condition (equation 16) together with the resource constraint (equation 5) determine the general equilibrium.

Proposition 1. A balanced growth path (BGP) in Regime $A$ exists if $0 \leq X \leq \tilde{X}$, $0<b<\frac{1}{1-\beta},(1-\beta) \rho X-F(\rho+\gamma)<\vartheta<(1-\beta) \rho X+\frac{F(\rho+\gamma)(1-b)}{b \beta}+\frac{1-b}{\beta}+b$, and $T \equiv \frac{1}{\gamma}>\widetilde{T}$ hold, where $\tilde{X}$ and $\tilde{T}$ define positive and finite threshold values. Assuming that $X \equiv \frac{V_{R}(t)-V_{P}(t)}{N(t)}$ remains constant when other parameters are changed, the following holds:

a) Along the BGP, an increase in the expected length of IP protection $T$ (三 $\frac{1}{\gamma}$ ) is associated with an increase in the rate of growth $g$ and with a reduction in the consumption share of poor households, $c_{P}$.

b) Along the BGP, $g$ depends negatively on $\vartheta$ and $F$ and positively on $\beta$ and $X$, so that inequality increases growth. $c_{P}$ depends positively on $\vartheta$ and negatively on $X$.

c) There is a positive threshold $\hat{X}<\tilde{X}$ such that for $0 \leq X<\hat{X}$, the BGP value of $g$ depends negatively (and $c_{P}$ positively) on the discount rate $\rho$. For $\hat{X}<X \leq \tilde{X}$, an increase in $\rho$ is associated with an increase in the rate of growth $g$ (paradox of thrift) and with a decrease in $c_{P}$.

Proof. See Appendix A1

An increase in $T$ (i.e. a fall in $\gamma$ ) makes research more profitable (for a given interest rate $r=\rho+g$ ) and increases the BGP growth rate. At the same time, it reduces the consumption share $c_{P}$ of poor households, while rich households always consume one unit of each good $\left(c_{R}=1\right)$. An increase in $T$ reduces the fraction $m$ of sectors in which IP protection has expired (see equation 8 ) and reduces the prices $p_{P}$ and $p_{R}$ at which IP protected goods are sold in equilibrium. While both rich and poor households are equally affected by the declines of $m$ and $p_{P}$, only the rich households benefit from the reduction in $p_{R}$ which allows them to purchase the same measure of goods as before. All the additional workers who move into the R\&D sector are therefore withdrawn from sectors that initially produce goods for all households and switch to exclusively serving rich households once $T$ is increased. It should be noted that these results are not merely driven by the resource constraint (equation 5) and the fact that R\&D uses labor as an input (and not some final composite good like in lab-equipment models) and also appear in a simple two-period version of the model in which incomes, marginal production costs and $R \& D$ costs are exogenously given (see Appendix B8). 
An increase in inequality that results from a reduction in $\vartheta$ or from an increase in $\beta$ increases the rate of growth. This result is a generalization of the result established by Föllmi and Zweimüller (2006) who (assuming that $\gamma=0$ ) only consider the case in which initial wealth is distributed in the same way as labor endowments $\left(\frac{V_{R}}{V_{P}}=\frac{l_{R}}{l_{p}}=\frac{1-\beta \vartheta}{(1-\beta) \vartheta}\right)$ and in which an exogenous change in $\beta$ or $\vartheta$ is accompanied by a corresponding exogenous change in $\frac{V_{R}}{V_{P}}$. If the wealth distribution becomes more unequal due to an increase in $X$, this increases the rate of growth, as it increases the expenditure difference between rich and poor households and allows innovators to raise prices ${ }^{32}$.

The paradox of thrift can arise for the following reason: an increase in the discount rate $\rho$ increases the consumption out of interest income and, for $X>0$, increases the expenditure difference between rich and poor households, which allows innovators to raise prices. If wealth inequality (i.e. $X$ ) is large, this effect is stronger than the standard discounting effect due to which a rise in $\rho$ discourages innovation, so that an increase in impatience can increase the rate of growth. Due to this mechanism, there might therefore be considerable growth in unequal economies with low savings propensities (like the US).

With the total labor income at date $t$ given by $N(t)$ and the total profit income given by $\pi(1-m) N(t)=F(\rho+g+\gamma)\left(\frac{g}{g+\gamma}\right) N(t)$ (equations 16 and 8 are used for the transformation), the labor income share can be derived as $S=\frac{g+\gamma}{g+\gamma+F g(\rho+g+\gamma)}$. As $\frac{\partial S}{\partial \gamma}>0$ and $\frac{\partial S}{\partial g}<0$, an increase in the length of IP protection (i.e. a reduction in $\gamma$ which increases $g$ ) reduces the BGP value of $S$.

\subsection{Transitional dynamics}

While the previous section (Proposition 1) compared BGPs assuming $X \equiv \frac{V_{R}(t)-V_{P}(t)}{N(t)}$ to be exogenously given, this section analyzes transitional dynamics, taking into account that $X$ might change when other parameters are changed.

Suppose that the economy is on a BGP and that at date $t=t_{0}$ there is an unexpected change in one (or several) of the exogenous parameters. An (unexpected) change in the length of IP protection is parametrized as follows: instead of expiring with hazard rate $\gamma$ like before $t_{0}$, IPRs granted after $t_{0}$ expire with hazard rate $\gamma_{1}$, while IPRs granted before $t_{0}$ that have not expired by $t_{0}$ expire with hazard rate $\gamma_{0}$ from $t_{0}$ onward. Then, the following proposition holds:

Proposition 2. Suppose that parameters are within the range defined by Proposition 1 so that a BGP in Regime A exists. Then, the transition following a change in an exogenous parameter takes the following form:

\footnotetext{
${ }^{32}$ In the case where $b=0$ ("digital economy" with zero marginal production costs), we obtain

$g=\frac{1}{F}$ (see equation 5) and $c_{P}=1-\frac{1-\vartheta+\rho X(1-\beta)}{1-F(\rho+\gamma)}$ along a BGP. While $g$ is independent of inequality in this case, $c_{p}$ still depends positively on $\gamma$ and $\vartheta$ and negatively on $X$. Poor households therefore still suffer from an increase in the length of IP protection (while rich households are indifferent) although it does not affect the rate of growth as all labor is inelastically supplied to the R\&D sector.
} 
a) $g$ and $c_{P}$ (and also $r, p_{R}$ and $p_{P}$ ) immediately jump to their new BGP values in $t_{0}$, while $m$ and $V$ adjust sluggishly (when $m>0$ ).

b) If $X=\frac{V_{R}(t)-V_{P}(t)}{N(t)}$ has the (constant) BGP value $X_{o}$ before $t_{0}$, it changes to $X_{1}=$ $\frac{\rho+g+\gamma_{1}}{\rho+g+\gamma_{0}} X_{o}$ (with $g$ indicating the new BGP value) in $t_{0}$ and stays constant at that level after $t_{0}$.

Proof. See Appendix A2.

If $\gamma_{0}=\gamma_{1}$ so that the length of IP protection is uniformly changed for newly granted and for existing IPRs in $t_{0}$, the value of initial wealth does not change in $t_{0}$. This is because the fraction $1-m$ of IP protected goods does not jump in $t_{0}$ and because the change in $\gamma$ is exactly offset by a change in $p_{R}, p_{P}$, and $g$ due to the free entry condition, implying that the value of an IPR remains constant at $Z=\frac{(1-\beta)\left(p_{R}-b\right)}{\rho+g+\gamma}=F$. Therefore, the distribution of initial wealth and $X$ do not change in $t_{0}$ in this case (unless there is an exogenous change in $X$ ). As $g$ and $c_{p}$ immediately jump to their new BGP values, the same comparative static results as in Proposition 1 (including the paradox of thrift) therefore hold if $\gamma_{0}=\gamma_{1}$ (or if parameters other than $\gamma$ are changed), even if transition dynamics are taken into consideration.

If the length of IP protection is only changed for newly granted IPRs, meaning that $\gamma_{1} \neq \gamma_{0}=\gamma$, the value of initial wealth $V$ and $X$ are multiplied by the factor $\left(\frac{\rho+g+\gamma_{1}}{\rho+g+\gamma}\right)$ in $t_{0}$ (see the proof of Proposition $2 ; g$ indicates the new BGP value). If $\gamma_{1}>\gamma$, so that the length of IP protection is decreased in $t_{0}$, the value of initial wealth and $X$ therefore increase. This is because an increase in $\gamma_{1}$ goes along with an increase in the prices $p_{R}$ and $p_{P}$ (and - given that $X$ is not too large - with a fall in $g$ and therefore with a fall in $r$ ), as new innovators could otherwise not break even. Holders of previously issued IPRs also benefit from this price increase (and potential fall in $r$ ) without being affected by the reduction in the length of IP protection, so that the value of their IPRs increases. While $g$ and $c_{p}$ still immediately jump to their new BGP values in $t_{0}$, this "valuation effect"33 implies that this jump might now differ from the comparative statics result derived in Proposition 1 in which $X$ was considered to be exogenous. While the "valuation effect" is negligible for small values of $X_{0}$ and therefore does not change the qualitative results of Proposition 1 in this case, the results can change if $X_{0}$ is sufficiently large.

Corollary 1. Suppose that $X_{o}=\frac{F}{1-\beta}$ (rich households own all initial wealth before $\left.t_{0}\right)$, $\vartheta=1$ (no inequality in labor income), $b=\frac{1}{2}$ and $F=\frac{\beta}{2 \rho}$. If, starting from a $B G P$ with infinite IP protection (i.e. $\gamma=0$; this BGP exists under the above conditions), the expected length of protection on new IPRs is slightly and unexpectedly reduced in $t_{0}$ (so that $\gamma_{1}>0$ ) while IPRs granted before $t_{0}$ are still infinitely lived (i.e. $\gamma_{0}=\gamma=0$ ), then the growth rate $g$ increases in $t=t_{0}$ to its new BGP value (and $c_{P}$ falls).

\footnotetext{
${ }^{33}$ It is assumed that households cannot insure against such policy-induced changes in the value of their wealth.
} 
Proof. See Appendix A3.

The explanation for this result is the following: when the length of IP protection is reduced for new innovations but not for old ones, the value of initial wealth increases due to the valuation effect just explained. As initial wealth is very unequally distributed and as $X$ is large in the case considered in Corollary 1 , the absolute increase in $X$ at $t=t_{0}$ is also large and implies a considerable increase in the expenditure difference between rich and poor households. This increase in inequality raises prices and incentives to innovate more than the reduction in the length of IP protection reduces these incentives, so that the rate of growth increases in this particular example ${ }^{34}$.

Proposition 2 also allows the study of the effects of a policy that unexpectedly "expropriates" owners of IPRs granted before $t_{0}$ by setting $\gamma_{0}>\gamma=\gamma_{1}$ in $t_{0}$. As the length of IP protection and therefore prices are unchanged for new innovations, the value of IPRs granted before $t_{0}$ decreases due to a faster rate of expiration $\gamma_{0}$. This leads to a one-time reduction in the value of initial wealth and of $X$ in $t_{0}$. According to Propositions $1 \mathrm{~b}$ and $2 \mathrm{~b}$, this reduction of wealth inequality caused by the expropriation leads to a reduction in the rate of growth in $t_{0}{ }^{35}$

\subsection{Regime B: uniform demand}

In this regime, poor households are so poor that they only consume goods with expired IPRs. This means that $C_{P}(t)<M(t)\left(c_{P}(t) \equiv \frac{C_{P}(t)}{N(t)}<m(t) \equiv \frac{M(t)}{N(t)}\right)$ and that IP holding firms face a "uniform" demand from rich households. A graphical representation of the equilibrium price and consumption structure is given in Figure 5.

\footnotetext{
${ }^{34}$ If the policy change was already announced to the households in $t_{-1}<t_{0}$, the valuation effect would already occur in $t_{-1}$ (but would be weaker the earlier $t_{-1}$ was relative to $t_{0}$ ). In this case, a reduction in the length of IP protection in $t_{0}$ might therefore lead to an increase in the rate of growth in $t_{-1}$, but not in $t_{0}$.

${ }^{35}$ The intuition for why $X$ stays constant along the transition (i.e. for $t>t_{0}$ ) is the following: Given a change in an exogenous parameter (for example a fall in $\gamma$ if $\gamma=\gamma_{1}=\gamma_{0}$ ) leads to a fall in $m$ during the transition, households anticipate that they need to increase their (normalized) expenditures $\frac{E_{i}}{N}$ in the future in order to be able to afford the same consumption shares $c_{i}=\frac{C_{i}}{N}\left(c_{P}<c_{R}=1\right)$. The reason for this is that $m$ is the fraction of non-IP protected goods that are sold at the lowest price $b$ and that a fall in $m$ implies that proportionally more goods are sold at price $p_{P}>b$ while still the same fraction $1-c_{P}$ of goods is supplied at price $p_{R}$ (note that due to Lemma $2 c_{P}$ and prices $p_{R}$ and $p_{P}$ are constant during the transition). Households who want to attain constant consumption shares $c_{i}$ therefore accumulate wealth in the transition phase and the total level of wealth, $V=N(1-m) F$, rises. As both rich and poor households consume the same measure $M=m N$ of cheap non-IP protected goods, they need to increase expenditures $E_{i}$ by the same absolute amount, which implies that they also need to increase their level of wealth $V_{i}$ (and the resulting interest income from which they finance the higher future expenditures) by the same absolute amount.

In the case where $m$ rises (for example due to an increase in $\gamma$ if $\gamma=\gamma_{1}=\gamma_{0}$ ), both rich and poor households reduce their (normalized) levels of wealth $\frac{V_{i}}{N}$ by the same absolute amount as they anticipate the same absolute fall in future expenditures. Therefore, $\frac{V_{R}-V_{P}}{N}=X$ holds during the whole transition period while $\frac{V_{R}}{V_{P}}$ and therefore wealth inequality changes. The effects of $\gamma$ on $g$ can therefore not be assessed if two BGPs along which $\frac{V_{R}}{V_{P}}$ is the same but $\gamma$ differs are compared.
} 
Proposition 3. A BGP in Regime $B$ exists if

$T \equiv \frac{1}{\gamma}<\tilde{T}=\frac{F\left(b-\vartheta-\rho \bar{V}_{P}\right)}{\left(\vartheta+\rho \bar{V}_{P}\right)\left(1-b(1-\beta)-\beta\left(\vartheta+\rho \bar{V}_{P}\right)\right)}<\infty$ (Condition $A$ is violated), $\vartheta+\rho \bar{V}_{P}<b$ and if $1-b(1-\beta)-\beta\left(\vartheta+\rho \bar{V}_{P}\right)>0$, where $\bar{V}_{P} \equiv \frac{V_{P}(t)}{N(t)}$ is constant along a BGP.

a) Along the $B G P, c_{P}=\frac{\vartheta+\rho \bar{V}_{P}}{b}$ and $g=\frac{1}{F}\left[1-b(1-\beta)-\beta\left(\vartheta+\rho \bar{V}_{P}\right)\right]$. Taking $\bar{V}_{P}$ as given, $g$ and $c_{P}$ are therefore independent of the expected length of IP protection $T$ (i.e. of $\gamma$ ). Higher inequality (lower $\vartheta$ or $\bar{V}_{P}$ or higher $\beta$ ) is associated with a larger rate of growth.

Suppose that the economy is on a BGP and that at date $t=t_{0}$ there is an unexpected change of IP protection (as described in Section 4.3) or of another exogenous parameter. Then, the transition takes the following form:

b) $g$ and $c_{P}$ (and also $r$ and $p_{R}$ ) immediately jump to their new BGP values while $m$, $V$ (total wealth) and $V_{R}$ adjust sluggishly.

c) If $\bar{V}_{P}(t)=\frac{V_{P}(t)}{N(t)}$ has the (constant) $B G P$ value $\bar{V}_{P}^{o}$ before $t_{0}$, it changes to $\bar{V}_{P}^{1}=$ $\frac{\rho+g+\gamma_{1}}{\rho+g+\gamma_{0}} \bar{V}_{P}^{o}$ (with $g$ indicating the new BGP growth rate) in $t_{0}$ and stays constant at that level after $t_{0} . g$ therefore increases in $t_{0}$ if $\gamma_{1}<\gamma_{0}$, which holds if IP protection is solely prolonged for newly issued IPRs in $t_{0}$.

Proof. See Appendix A4.

This regime is therefore more likely to arise the shorter IP protection $T$ is (i.e. the larger $\gamma$ is) and the poorer the poor households are (i.e. the lower $\vartheta$ and $\bar{V}_{P}$ are; adding more poor income groups with $C_{i}<M$ would not change any of the qualitative results). The rate of growth in Regime $B$ therefore only depends on the length of IP protection if a change in the latter affects the level of wealth of poor households and therefore the distribution of income and expenditures. If $\gamma_{1}<\gamma_{0}$, either because in $t_{0}$ IP protection is solely prolonged for new innovations $\left(\gamma_{1}<\gamma=\gamma_{0}\right)$ or because it is reduced for IPRs issued before $t_{0}\left(\gamma_{1}=\gamma<\gamma_{0}\right.$, i.e. "expropriation"), the value of initial wealth held by poor households declines due to the valuation effect described above and their consumption decreases $^{36}$. As rich households always purchase one unit of each good, the total demand for production labor therefore decreases, leaving more labor for R\&D (see equation 5) so that the rate of growth increases.

If $\gamma_{1}=\gamma_{0}$ or if $\bar{V}_{P}^{o}=0$, there is no valuation effect and a change in the length of IP protection $T$ neither affects the level of initial wealth of poor households and their consumption nor growth ${ }^{37}$. This can also be understood by looking at the demand side: a reduction in $T$ (i.e. an increase in $\gamma_{1}=\gamma_{0}$ ) on the one hand reduces the value of an innovation by reducing the expected time span during which an innovator has monopoly

\footnotetext{
${ }^{36}$ Poor households spend $E_{P}(t)=\vartheta N(t)+\rho V_{P}(t)$ per period and consume the measure $C_{P}(t)=\frac{E_{P}(t)}{b}$ of non-IP protected goods that are sold at price $b$.

${ }^{37}$ Note that in the case where $\bar{V}_{P}^{o}=0, g$ is independent of $\rho$. This is because $\rho$ does not affect the value of an innovation in this case as the increase in discounting implied by an increase in $\rho$ is exactly offset by an increase in markups.
} 
power, but on the other hand leads to an increase in the price $p_{R}$ and in per period monopoly profits ${ }^{38}$. When $\gamma_{1}=\gamma_{0}$ or $\bar{V}_{P}^{o}=0$, the two effects exactly offset each other, leaving the incentives to innovate and the rate of growth unchanged. While a change in $T$ changes the fraction $1-m$ of sectors in which IPRs have not expired and the price $p_{R}$ at which IP protected goods are sold, it does not change the value of initial wealth held by rich households in $t_{0}$ if $\gamma_{1}=\gamma_{0}$ or if $\bar{V}_{P}^{o}=0$. As only rich households consume IP protected goods in Regime $B$, such a change in $T$ therefore merely shifts demand across sectors and time but does not affect "total demand" and the profitability of R\&D. Because of that, firms find it optimal to hire the same amount of R\&D workers as before, leaving the rate of growth unchanged. An increase in the length of IP protection (i.e. a reduction in $\gamma_{1}=\gamma_{0}$ ) therefore only leads to an increase in growth if it is sufficiently strong to push the economy from Regime B to Regime $\mathrm{A}^{39}$. Like in Regime A, these results not merely driven by the resource constraint (equation 5) and can also be obtained in a simple two-period model in which incomes, marginal production costs and R\&D costs are exogenously given (see Appendix B8) ${ }^{40}$.

The result that $g$ is independent of $\gamma_{1}=\gamma_{0}$ also holds in Regime $\mathrm{A}$ if there is no inequality $(\beta=0$ or $\vartheta=1$ ). When $\beta$ and therefore the level of inequality is small, reducing $\gamma_{1}=\gamma_{0}$ can only have a small positive effect on $g$ (see equation 5). Prolonging IP protection (in a uniform way) is therefore more likely to increase the rate of growth substantially if the distribution of income across households that consume IP protected goods is sufficiently unequal. This is the first main result of the paper ${ }^{41}$.

As in Regime A, the labor income share along a BGP is given by $S=\frac{g+\gamma}{g+\gamma+F g(\rho+g+\gamma)}$.

\footnotetext{
${ }^{38}$ This is different in models with CES preferences, where monopolists always charge the same markups, independently of $m$. Deneckere and Judd (1992) and Matsuyama (1999) (who assume CES preferences and study endogenous innovation cycles) actually focus on the opposite case where the elasticity of substitution between goods is so high that an increase in the fraction of competitively supplied goods $(m)$ reduces demand for IP protected goods and (ceteris paribus) the per period profits of IP holding firms.

${ }^{39}$ If a marginal change in an exogenous parameter leads to a switch between Regimes B and A, this does not lead to a discontinuous jump in $c_{P}$ or $g$, implying that Propositions 2 and 3 cover the whole admissible parameter range without any discontinuities arising at the switching point. The reason for this is the following: as firms are indifferent about selling an IP protected good at the high price $p_{R}$ to only rich consumers or at the lower price $p_{P}$ to both rich and poor consumers, the value of an innovation and of the level of initial wealth do not change in a discontinuous way if poor consumers can either afford to purchase a small amount of IP protected goods (Regime A) or no IP protected goods at all (Regime B). Because of that, there is no discontinuity in the incentives to undertake R\&D and in the equilibrium rate of growth $g$. This is different in Hatipoglu (2012) where markups are assumed to be the same, independently of the number of households who purchase a good.

${ }^{40}$ In this model, there are, however, no valuation effects as there is no initial wealth

${ }^{41}$ If there was an intensive margin in addition to the extensive margin of consumption, an increase in $m$ and an increase in prices of IP protected goods caused by a reduction of $T$ would induce households to consume less units of each IP protected good and more units of non-IP protected goods. This would reduce "total demand" for IP protected goods and the incentives to innovate, even if there was no inequality. This effect would, however, be weaker the faster households get satiated with individual goods if they consume more of them (with the limit being the case of 0 - 1 consumption analyzed here) and would probably not change the qualitative result that an increase in the length of IP protection increases growth more if there is inequality among households consuming IP protected goods.
} 
A reduction in the length of IP protection (i.e. an increase in $\gamma$ ) increases $S$ even though it does not affect $g$. This is because an increase in $\gamma$ implies that innovators earn higher profits (due to higher markups), but for a shorter expected duration $T$, so that there is less discounting (note that $r$ is constant when $g$ is constant), implying that a lower total profit income is needed in order to provide the same innovation incentives.

\subsection{Welfare analysis}

Taking into account that $\int_{j=0}^{\infty} c_{R}(j, t) d j=C_{R}(t)=N(t)$ and that $\int_{j=0}^{\infty} c_{P}(j, t) d j=C_{P}(t)=$ $c_{P} N(t)$, intertemporal utilities (equation 1) along a balanced growth path along which the consumption share $c_{p}\left(\equiv \frac{C_{P}(t)}{N(t)}\right)$ of poor households is constant and along which $N(t)$ grows at the constant rate $g$ can be derived as:

$$
\begin{gathered}
U_{R}(\tau)=\int_{t=\tau}^{\infty} \ln (N(t)) e^{-\rho(t-\tau)} d t=\frac{\ln (N(\tau))}{\rho}+\frac{g}{\rho^{2}} \\
U_{P}(\tau)=\int_{t=\tau}^{\infty} \ln \left(c_{P} N(t)\right) e^{-\rho(t-\tau)} d t=\frac{\ln (N(\tau))}{\rho}+\frac{\ln \left(c_{P}\right)}{\rho}+\frac{g}{\rho^{2}}
\end{gathered}
$$

As there are no transitional dynamics for $c_{P}$ and $g$ (see Propositions $2 \mathrm{a}$ and $3 \mathrm{~b}$ ), it suffices to compare intertemporal utilities along balanced growth paths in order to evaluate welfare effects of different policies.

Proposition 4. a) Rich households benefit from any change in IP (or redistributive) policy that increases the rate of growth. Poor households benefit from changes in IP (or redistributive) policies that increase the rate of growth if $c_{P}>\frac{F \rho}{b \beta}$ (case $A$ ) and are harmed by them if $c_{P}<\frac{F \rho}{b \beta}$ (case B). Case $B$ always arises if $\frac{F \rho}{b \beta}>1$. If $\frac{F \rho}{b \beta}<1$, Case $B$ arises under the conditions leading to Regime $A$ if $\vartheta<\frac{F \rho}{b \beta}(1+F \gamma)+\rho X(1-\beta)-F \gamma$ and under the conditions leading to Regime $B$ if $\vartheta<\frac{F \rho}{\beta}-\rho \bar{V}_{P}$.

b) Suppose that the expected length of IP protection can only be changed in a uniform way, affecting new and old innovations symmetrically $\left(\gamma=\gamma_{0}=\gamma_{1}\right)$. Then, there is a non-empty range of parameters for which poor households prefer a finite expected length of IP protection equal to $T_{P}^{*}=\frac{1}{\gamma_{P}^{*}}=\frac{F\left(1-\frac{F \rho}{b \beta}\right)}{\frac{F \rho}{b \beta}-\vartheta+\rho X(1-\beta)}<\infty$ and for which the economy is in Regime $A$. $T_{P}^{*}$ decreases in $X$ and $\rho$ and increases in $\vartheta$ and in $\beta$.

Proof. See Appendix A5.

As rich households always consume one of each of the invented goods $\left(C_{R}(t)=N(t)\right)$, they benefit from any policy that increases the rate of growth $\left(g=\frac{\dot{N}(t)}{N(t)}\right)$ as it increases 
their future consumption without affecting their current consumption. For poor households, there is, however, a trade-off as an increase in $T$ (i.e. a reduction in $\gamma=\gamma_{1}=\gamma_{0}$ ) or $X$ or a reduction in $\vartheta$ on the one hand increases $g$ (dynamic effect) but on the other hand reduces their consumption share $c_{P}$ (static effect). While poor households might benefit from growth-enhancing policies if the equilibrium level of $c_{P}$ is relatively large ${ }^{42}$, they are harmed by them if $c_{P}$ is relatively low, which can for example be the case if $\vartheta$ is sufficiently low or if $X$ is sufficiently high (or if $\bar{V}_{P}$ is sufficiently low, when in Regime B).

Given that $\gamma=\gamma_{1}=\gamma_{0}$ (so that an increase in $T$ increases $g$ and does not affect $X$ ) and that an equilibrium in Regime A exists in which the length of IP protection preferred by poor households, $T_{P}^{*}$, is finite, the comparative statics results of Proposition $4 \mathrm{~b}$ arise for the following reason: the level of $c_{P}$ that optimally trades off static and dynamic welfare effects and maximizes the intertemporal utility of poor households is given by $c_{P}=c_{P}^{*}=\frac{F \rho}{b \beta}$ and therefore independent of $T$ (i.e. of $\gamma$ ), $\vartheta$ and $X$. When $T$ is set equal to $T_{P}^{*}$ in order to attain this outcome, $T_{P}^{*}$ therefore has to decrease in $X$ and to increase in $\vartheta$ as $c_{P}$ decreases in $X$ and $T$ and increases in $\vartheta$ (see Propositions $1 \mathrm{a}$ and $1 \mathrm{~b}$ and Proposition 2). When $\rho$ increases (households get more impatient), current consumption becomes more important relative to growth $\left(c_{P}^{*}\right.$ increases) and this effect is so strong that, independently of how $\rho$ affects $c_{P}$ for a given $T$, poor households want to reduce $T$ in order to reduce growth. When $\beta$ increases, $c_{P}^{*}$ decreases and poor households prefer a larger rate of growth ${ }^{43}$. While an increase in $\beta$ already increases $g$, the first effect is so strong that poor households want to increase $T$ in order to increase $g$ further.

Given that $c_{P}<\frac{F \rho}{b \beta}$, so that (if $\gamma=\gamma_{0}=\gamma_{1}$ ) poor households want to reduce $T$ (i.e. to increase $\gamma$ ) while rich households want infinite IP protection $(\gamma=0)$, there is no efficient length of IP protection which can make both groups better off, even if income or wealth transfers that affect $\vartheta$ and $X$ are permitted. The reason for this is simply that (for $\beta$ given) rich households can only benefit if $g$ increases, which (due to the resource constraint) implies that $c_{P}$ has to decrease, making poor households worse off ${ }^{44}$. In order to "buy" the support of poor households for longer IP protection, rich households would therefore have to agree to transfers that increase $\vartheta$ or reduce $X$ so much that the positive growth effect caused by the increase in $T$ would be completely offset, destroying any

\footnotetext{
${ }^{42}$ Föllmi and Zweimüller (2006) have already shown that poor households can benefit from a regressive transfer which reduces $\vartheta$ (they assume that there is at the same time a proportional increase in wealth inequality).

${ }^{43}$ From the resource constraint (equation 5 ) it can be inferred that the preferred growth rate is given by $g_{P}^{*}=\frac{1}{F}\left[1-b \beta c_{P}^{*}-b(1-\beta)\right]=\frac{1}{F}[1-F \rho-b(1-\beta)] . g_{P}^{*}$ increases in $\beta$ for the following reason: if $\beta$ increases, a given reduction in $c_{P}$ frees more resources that can then be used for $\mathrm{R} \& \mathrm{D}$ (the benefits of which accrue to all households due to its public good nature).

${ }^{44}$ In Appendix B2 it is shown that a social planner who maximizes a weighted sum of intertemporal utilities and puts welfare weight $\alpha>\frac{1}{2}$ on rich households and welfare weight $1-\alpha$ on poor households finds it optimal to set $C_{P}(t)<C_{R}(t)=N(t)$ and to increase $g$ (and to therefore reduce $C_{P}$ and $c_{P}$ ) when $\alpha$ increases given that $\frac{\alpha F \rho}{b(\alpha+\beta-2 \alpha \beta)} \geq 1>\frac{(1-\alpha) F \rho}{b(\alpha+\beta-2 \alpha \beta)}$ holds.
} 
welfare gain for rich households ${ }^{45}$.

Given that $c_{P}<\frac{F \rho}{b \beta}$, poor households might want to reduce $T$ (i.e. to increase $\gamma=\gamma_{0}=\gamma_{1}$ ) and to therefore increase $m=\frac{\gamma}{g+\gamma}$ (note that $m$ increases in $\gamma$ if $g$ declines in $\gamma)$ so much that Condition $\mathrm{A}\left(c_{P}>m\right.$, implying that $\left.T>\tilde{T}\right)$ is violated, leading to a switch to Regime $\mathrm{B}^{46}$. As the economy remains in this regime for any $T<\tilde{T}$, and as $g$ is independent of $T$ in this regime (assuming $\gamma=\gamma_{0}=\gamma_{1}$ ), poor households are then indifferent with respect to the exact value of $T<\tilde{T}$.

\subsection{Many income groups}

Suppose there are $k$ income groups indexed by $i$ with labor endowments $l_{i}$, initial wealth $V_{i}$ and population shares $\beta_{i}\left(\sum_{i=1}^{k} \beta_{i}=1\right)$. In order to simplify the analysis it is assumed that households in all income groups have the same (absolute) amount of initial wealth, so that $V_{i}(t)=V(t)$. In this case, all households are affected symmetrically when there is a change in $V$ resulting from an unexpected change in the length of IP protection that affects newly granted and previously issued IPRs differently $\left(\gamma_{0} \neq \gamma_{1}\right)$. This implies that the expenditure differences between households with different incomes are not affected by valuation effects and that the rate of growth only depends on the hazard rate $\gamma=\gamma_{1}$ with which newly granted IPRs expire, but not on the rate $\gamma_{0}$, with which previously issued IPRs expire.

Groups are ordered by income (labor endowment), so that households in group 1 are the poorest and households in group $k$ the richest $\left(l_{1}<l_{2}<\ldots,<l_{k-1}<l_{k}\right)$. The size of the population and the total labor endowment are normalized to one (so that

\footnotetext{
${ }^{45}$ This result, however, needs to be qualified. In a previous version of the paper (Kiedaisch, 2009), a more general setup was studied in which instantaneous utility is given by $u_{i}\left(x_{i}(t), c_{i}(j, t)\right)=$ $x_{i}(t)^{\nu} \int_{j=0}^{\infty} c_{i}(j, t) d j$, with $x_{i}(t)$ denoting either the variety or the quantity (assuming divisibility) of noninnovative, competitively supplied goods that a household consumes (one unit of these goods can be produces using $b_{x}$ units of labor) and $\nu \geq 0$ indicating the consumption value of these goods relative to innovative goods. Within this setup (assuming $X=0$ and $\nu>0$ and considering the regime in which $\left.M(t)<C_{P}(t)<C_{R}(t)=N(t)\right), x_{i}(t)$ depends positively on the price of the most expensive good $j$ that household $i$ consumes (so that $x_{R}(t)>x_{P}(t)$ ) and therefore falls when $T$ is larger, i.e. when there is a larger fraction of IP protected goods sold at lower prices. Unlike in the case where $\nu=0$, rich households therefore also suffer from a loss in current consumption (due to a reduction in $x_{R}(t)$ ) when $T$ increases, but still prefer longer IP protection than poor households who suffer from a reduction in both $x_{P}$ and $c_{P}$. Given that $T$ is equal to the length of IP protection preferred by poor households, it is then possible that both poor and rich households benefit from increasing $T$ if rich households at the same time compensate poor households with transfer payments (that might take the form of an increase in $\vartheta$ ). The reason for this is that increasing $T$ reduces the consumption levels $x_{i}(t)$ of the non-innovative goods (that are inefficiently high when IP protected goods are sold at a price that exceeds the marginal production costs) and thereby leads to a reallocation of labor from the production of these goods into the R\&D sector, rendering infinite IP protection efficient.

${ }^{46}$ The threshold level at which this switch occurs and at which $c_{P}=m$ holds is implicitly defined by $\widetilde{T}=\frac{1-c_{P}(\tilde{\gamma})}{g(\tilde{\gamma}) c_{P}(\tilde{\gamma})}$. When $T$ is low (i.e. when $\gamma$ is high) a BGP in Regime A therefore more likely exists if parameters are such that the equilibrium values of $g$ and $c_{P}$ are large.
} 
$\left.\sum_{i=1}^{k} \beta_{i} l_{i}=1\right)$. Households belonging to the richest income group $k$ again consume one of each of the invented goods along a BGP, so that $C_{k}(t)=N(t)$ and $c_{k}(t)=\frac{C_{k}(t)}{N(t)}=1$. Households belonging to a poorer group $i<k$ only consume the fraction $c_{i}(t)=\frac{C_{i}(t)}{N(t)}$ of the goods, with $c_{i}(t)$ increasing in $i$. IP protected goods are again sold at different prices which are inversely related to the number of income groups that purchase a particular good.

Proposition 5. a) Suppose that $V_{i}=V$ and that a BGP exists in which even the poorest households consume some IP protected goods, so that $c_{1}>m$ holds (this requires that $T$ is sufficiently large). Then, the following holds:

i) An increase (a decrease) in the expected length of IP protection T resulting from a reduction in $\gamma=\gamma_{1}$ increases (decreases) the rate of growth $g$ and reduces (increases) the consumption shares $c_{i}(t)=\frac{C_{i}(t)}{N(t)}$ of all households except for those belonging to the richest group. The poorer a household is, the larger is the absolute reduction (increase) in $c_{i}$.

ii) $g(t)$ and $c_{i}(t)$ immediately jump to their new BGP values when $T$ is changed.

iii) The richest households $(i=k)$ always prefer infinite IP protection. Given that the intertemporal utility of a household belonging to group $n(1<n<k)$ is maximal for a finite expected length of IP protection $T_{n}^{*}$ for which a BGP with $c_{1}>m$ exists, all poorer households $(i<n)$ benefit from a reduction of $T$ below the level $T_{n}^{*}$ and are harmed by an increase of $T$ above the level $T_{n}^{*}$, while the opposite holds for all richer households $(i>n)$.

Proof. See Appendix A6.

The intuition for these results ${ }^{47}$ is the following: when $T$ increases, $m$ falls and prices $p_{i}$ of IP protected goods fall $\left(p_{i}\right.$ denotes the price of goods that are still consumed by households belonging to group $i$ but not by poorer households ${ }^{48}$ ). While all households consume all the $M(t)=m(t) N(t)$ goods on which IP protection has expired and - taking expenditures as given - suffer from a reduction in $m$, the effects of the fall in prices $p_{i}$ affect poor and rich households differently: For a given expenditure difference (which does not depend on $\gamma$ if $V_{i}=V$ ), the consumption difference between a rich and a poorer household increases as the rich one benefits from a reduction in prices of a subset of IP protected goods that the poorer one cannot afford to buy. Taking into account that the fall in $p_{i}$ is so large that it allows households in the richest income group to continue consuming one of each of the invented goods, this implies that the reduction in the consumption share (and in $C_{i}$ ) is largest (in absolute terms) for the poorest households $(i=1$ ) that only benefit from the reduction in $p_{1}$ but not from the reduction in any other prices $p_{i}$ with

\footnotetext{
${ }^{47}$ The following additional result can be derived: if income is redistributed in such a way that $l_{n+1}-l_{n}$ increases for some $i=n$ and does not decrease for any other $i \neq n, g$ increases (see Appendix B6).

${ }^{48} \mathrm{~A}$ fall in $p_{i}$ therefore only implies that prices of IP protected goods fall conditional on demand, but not that prices for all IP protected goods fall, as some firms increase prices and sell to less households when IP protection is increased.
} 
$i>1$. Consequently, poorer households are more likely to benefit from a reduction in $T$ and prefer a shorter duration of IP protection, while the richest households always prefer IP protection of infinite duration $(\gamma=0)$. This is the second main result of the paper.

Given that $\gamma=\gamma_{1}=\gamma_{0}$, so that there are no valuation effects when the length of IP protection $T$ is changed, no household belonging to income group $i$ ever wants to reduce $T$ beyond the point where $m$ starts to exceed the own consumption share $c_{i}$. The reason for this is that (absent valuation effects) households with $c_{i}<m$ always benefit from an increase in $T$ as it increases the rate $g$ with which their wages grow ${ }^{49}$ without reducing their own consumption share. The result that poorer households prefer shorter IP protection therefore only holds for "middle class" households that consume some IP protected goods, but not for very poor households that do not consume any of these $\operatorname{goods}^{50}$.

\subsubsection{Application to an international context}

Suppose that the model above describes the world economy and that households belong to different countries $l$. This requires the strong assumptions that there are no trade costs, that spillovers are global and that, due to the possibility of parallel trade, each good is sold at the same price in each country. Then, the analysis implies that poorer countries (or countries in which the median voter who is pivotal for policy decisions is poorer) prefer a (weakly) shorter duration of international IP protection than richer ones. If countries set their IP policies $\gamma_{l}$ independently, the effective hazard rate of IP expiration is given by $\gamma=\sum_{l} \gamma_{l}$ and the expected duration of IP protection by $T=\frac{1}{\sum_{l} \gamma_{l}}$ as, due to the possibility of parallel trade, the price of a good drops to marginal cost whenever the IPR expires for the first time in one of the countries. If every country can increase the effective global expiration rate $\gamma$ by raising $\gamma_{l}$ as much as it wants, the equilibrium level of $T$ is equal to the shortest one any of the countries prefers when IP policies are set in a non-cooperative way. Poor countries are then only willing to voluntarily join an international agreement that sets minimal standards for the protection of intellectual property rights (like TRIPS or TPP) if they are compensated appropriately by richer countries who prefer imposing a larger $T$.

Suppose now that parallel trade between countries is prohibited so that innovators

\footnotetext{
${ }^{49}$ While a change in $T$ can only affect $g$ through the channel of valuation effects if there is only one income group that consumes IP protected goods (see Proposition 3), an increase in $T$ (assuming $\gamma=\gamma_{1}=\gamma_{0}$ ) always increases $g$ if IP protected goods are bought by more than one income group.

${ }^{50}$ The result that very poor households benefit from an increase in $T$ arises due to the assumption that R\&D leads to knowledge spillovers that increase the productivity of labor. This assumption was made in order to allow for balanced growth (see footnote 19) but might not be very realistic. The result that poorer "middle class" households with $c_{i}>m$ prefer weaker IP protection (i.e. a lower $T$ ) is, however, more robust and also holds in a simple two-period version of the model without spillovers which is analyzed in Appendix B8. In this simple model, the utility of households with $c_{i}<m$ is independent of the strength of IP protection.
} 
can set different prices in different countries and can earn profits in a country as long as the IPR is protected there, independent of whether it has expired in another country. Given that the richest households in a country are sufficiently rich $^{51}$, prices are then set in such a way in this country that these households can afford to consume one of each of the goods in equilibrium. Poorer households in the same country then just consume a fraction of the goods. As an increase in the length of IP protection in one country increases the (global) incentives to undertake $\mathrm{R} \& \mathrm{D}$ and growth, the richest households in a country who consume all of the goods therefore always prefer infinite IP protection in their country, while poorer households again face a trade-off between an increase in the rate of growth and a reduction in their current consumption and might prefer a shorter duration of IP protection.

\section{Extension: restricted monopoly power}

In this section, the basic model is extended by one additional feature: it is assumed that any good $j(j \epsilon[0 ; \infty))$ can be produced using a traditional, inefficient, technology, so that an innovation merely allows to produce a given good $j$ at lower marginal cost (process innovation) but does not lead to the invention of a new good variety (product innovation). Producing one unit of a good using the traditional technology is assumed to require $\frac{\Omega}{N(t)}$ units of labor input, where $\Omega>b \geq 0$ holds. It is assumed that traditional technologies are in the public domain and that they are competitively supplied at the marginal cost of $\Omega\left(=w(t) \frac{\Omega}{N(t)}\right)$. As there is an infinite supply of symmetric goods at the price of $\Omega$, a household's willingness to pay for a single good $j$ never exceeds $\Omega$, so that $z_{P}(t) \leq z_{R}(t) \leq \Omega$ must hold ${ }^{52}$. The presence of the traditional technology therefore prevents IP holding innovators from charging a price in excess of $\Omega$. When $\Omega$ is large, this restriction is never binding, as IP holding firms find it optimal to charge a price that lies below $\Omega$. Then, the analysis is the same as in the main part of the paper. If the traditional technologies are more efficient, i.e. if $\Omega$ is lower, IP holding firms might, however, be constrained in their price setting power and the following new "constrained" regimes can arise ${ }^{53}$ :

C1): $\left.C_{P}(t)<N(t)<C_{R}(t), \mathbf{C 2}\right): C_{P}(t)=N(t)<C_{R}(t)$ and C3): $N(t)<C_{P}(t)<$ $C_{R}(t)$.

In the following, the main insights obtained from studying these regimes are briefly

\footnotetext{
${ }^{51}$ If these households are so poor that they cannot afford to purchase all existing goods, even if they are sold at marginal cost, IP holders cannot earn any profits in this country, as competition between producers of different goods drives their prices down to marginal cost. The extent of IP protection $\left(\gamma_{l}\right)$ in such a country is then irrelevant for economic outcomes.

${ }^{52}$ The traditionally produced goods might also be interpreted as home-produced goods or as leisure time.

${ }^{53}$ Bertola, Föllmi and Zweimüller (2006, Section 11.2.2) study a similar setup assuming infinite IP protection $(\gamma=0)$.
} 
summarized. The formal derivations are provided in Appendix B7. The focus of the analysis lies on Regime $\mathrm{C} 1$ in which, in line with the empirical evidence, poorer households purchase a smaller variety of "innovative goods" than richer households do.

\subsection{Regime C1: limit pricing}

In this regime, rich households consume some traditionally produced goods and some exclusively sold IP protected goods at the limit price $\Omega$. Considering the case where $M(t)<C_{P}(t)$, part of the IP protected goods are sold to both income groups at price $p_{P}(t)=z_{P}(t)=\beta b+(1-\beta) \Omega$ (see Figure 6 for a graphical representation). A uniform increase in $T$ (i.e. a decline in $\gamma=\gamma_{1}=\gamma_{0}$ ) increases $g$ and, unlike in regimes $\mathrm{A}$ and $\mathrm{B}$ in which markups depend negatively on $T$, reduces $C_{P}$ and $C_{R}$ by the same absolute amount. While rich households always prefer infinite IP protection, poor households prefer a finite length of protection $\left(T_{P}^{*}<\infty\right)$ if inequality is sufficiently large (and infinite protection otherwise). The reason for this is that intertemporal utility is concave in $C_{i}$ and linear in $g$, making poorer households (with a lower $C$ ) more willing to give up on growth in order to increase current consumption ${ }^{54}$

\subsubsection{Length vs breadth of IP protection}

Suppose now that policymakers can restrict the maximal price that IP holding firms can charge to a level $\bar{p}<\Omega$. This might be done by reducing the breadth of IP protection by allowing entrants to supply inferior imitations of IP protected goods as long as their marginal production costs do not lie below $\bar{p}$. A lower breadth of IP protection then forces IP holders who sell exclusively to rich households to charge a lower limit price $\bar{p}$ in order to prevent entry. Alternatively, antitrust authorities might directly impose a price cap $\bar{p} .{ }^{55}$

Then, rich households prefer long (large $T$ and low $\gamma$ ) and narrow (low $\bar{p}$ ) IPRs while poor households prefer short (low $T$ and high $\gamma$ ) and broad (high $\bar{p}$ ) IPRs in order to provide innovation incentives that lead to a given rate of growth. The intuition behind this result is the following: an increase in $T$ reduces $m$ and thereby reduces both $c_{P}$ and $c_{R}$ by the same absolute amount, as households in both income groups consume all of the goods on which IP protection has expired (note that prices of IP protected goods do not depend on $T$ in this regime). Contrary to that, an increase in $\bar{p}$ reduces $c_{R}$ more than $c_{P}$, as it implies that rich households not only have to pay higher prices for the

\footnotetext{
${ }^{54}$ This does not happen in models with CES preferences where (unlike in Saint - Paul (2006)) rich and poor households face the same trade-off between growth and current consumption and where an increase in the fraction of monopolistic firms (i.e. a reduction in $m$ ) reduces current consumption of all households proportionally as the amount of each good that a household consumes is proportional to household expenditures.

${ }^{55}$ Another reason for a price restriction might be that IP holders are forced to license their innovation to competitors at the fixed per unit royalty rate $\bar{p}$.
} 
IP protected goods that are purchased by all households, but also for the IP protected goods that they consume exclusively. Consequently, rich households prefer increasing IP protection by increasing $T$, while poor households prefer to increase it by increasing $\bar{p}$, shifting the burden towards rich consumers.

\subsection{The effect of IP protection on the relation between inequality and growth}

In Regime C2 (see Figure 7), the length of IP protection $T$ has no effect on $g$ when $\gamma_{1}=\gamma_{0}$, as it (like in Regime B) leaves "total demand" unaffected and merely shifts the uniform demand across sectors and time. In Regime C3 (see Figure 8), increasing $T$ increases $g$ and reduces $c_{P}$ and $c_{R}$ by the same absolute amount, and both rich and poor households always benefit from an increase in $T$.

The effect that inequality has on growth differs in the three regimes: in Regime $\mathrm{C} 1$, $g$ depends negatively on $\beta$ and does not depend on $\vartheta$ and $X$; in Regime $\mathrm{C} 2, g$ depends positively on $\vartheta$ and negatively on $X$ (inequality reduces growth) and in Regime C3, inequality has no effect on growth.

Suppose that parameters are such that Condition $\mathrm{A}\left(C_{P}>M\right)$ always holds and that the economy is in Regime A (in which inequality increases growth) for high values of $T$. Then, a reduction in $T$ (starting from $T=\infty$ ) first leads to a shift to Regime $\mathrm{C} 1$, then to a shift to Regime C2, and finally to a shift to Regime C3. Therefore, a reduction in the length of IP protection makes it less likely that inequality increases growth. The same holds true for a reduction in patent breadth. The intuition behind this result is that a reduction in IP protection makes it more likely that firms become constrained in their price setting power and that the incentives to innovate primarily depend on the size of the market (which tends to be larger when inequality is lower) and not on individual households' willingness to pay in this case.

Dorn and Kiedaisch (2017) analyze a static (two-period) version of the model with a continuous income distribution and also find that reducing patent protection makes it less likely that inequality increases innovation or more likely that it decreases innovation. Studying the variation in international patent applications, they moreover provide empirical evidence indicating that this effect might indeed be at work.

\section{Conclusion}

This paper has studied the effects that increasing the (expected) length of intellectual property protection has on inequality and growth in a model in which, in accordance with empirical evidence, rich households consume a larger variety of goods than poorer ones. 
The first main result of the analysis is that, unlike in models with homothetic preferences, the effects that IP protection has on growth depend on the distribution of income. While a (uniform) increase in the length of IP protection increases growth when there is inequality among households consuming IP protected goods, it does not when there is no such inequality. Moreover, an unexpected decline in the length of IP protection for new, but not for previously issued IPRs can increase growth by increasing inequality.

The second main result is that poor households who consume fewer IP protected goods than richer ones prefer a shorter length of IP protection than richer ones if increasing the length of IP protection increases growth. This is because richer households benefit more from a fall in markups of IP protected goods when the length of IP protection is increased while all households are harmed in a similar way from the resulting reduction in the number of competitively supplied goods. While the existing literature has focused on channels through which increased IP protection can increase income inequality by increasing the skill premium or the rate of return on assets, this paper therefore identifies a new channel through which it can increase consumption inequality and through which new conflicts of interest can arise that are not present in standard growth models with homothetic preferences.

When firms' price setting power is restricted, it is, moreover, shown that rich (poor) households prefer long and narrow (short and broad) IP protection and that an increase in inequality is more likely to increase growth the stronger IP protection is.

While it might in many cases be more efficient to address distributional concerns by using taxes and transfers and not by changing IP legislation, this analysis has identified several new mechanisms, the understanding of which might help design policies conductive to equitable growth.

In order to simplify the analysis, it was assumed that households either consume zero or one units of any given good. While the considerable differences in the varieties of goods purchased by rich and poor households found in the data indicate that households do quickly become satiated with individual goods and/or that consumption indivisibilities are important, it would clearly be interesting to make the analysis more general by allowing for intensive consumption margins for all goods ${ }^{56}$. Moreover, the model could be extended by allowing for (cumulative) quality-increasing or cost-reducing innovations. These extensions are left for future research.

\footnotetext{
${ }^{56}$ In a previous version of the paper (Kiedaisch, 2009), I have studied a more general setup in which households can consume a homogenous (divisible) outside goods in addition to the non-divisible innovative goods. Some of the effects that arise in this setup are likely to also arise in a more general setup with intensive consumption margins for all goods (for a brief description of these effects, see footnotes 45 and the discussion of Proposition 8 in Appendix B7).
} 


\section{References}

Adams, Samuel (2008); "Globalization and income inequality: Implications for intellectual property rights", Journal of Policy Modeling, 30, pp. 725-735

Aghion, P., Akcigit, U., Bergeaud, A., Blundell, R, and D. Hémous (2016), "Innovation and Top Income Inequality", working paper

Barro, R. J. and X. Sala-i-Martin (2004), "Economic Growth", (second ed.), MIT Press, Cambridge, MA

Beerli, A., F. Weiss, F. Zilibotti and J. Zweimüller (2014); "Demand Forces of Technical Change: Evidence from the Chinese Manufacturing Industry", working paper

Bernal Uribe, Juan Felipe (2012); "Innovation, Intellectual Property Rights and International Knowledge Diffusion", Chapter 1, Ph.D. dissertation, Toulouse School of Economics

Bertola, Giuseppe, Föllmi, Reto and Josef Zweimüller (2006); "Income Distribution in Macroeconomic Models", Princeton University Press

Cai, Dapeng and Takashi Gyoshin Nitta (2012); "Transversality Conditions for Stochastic Higher-Order Optimality: Continuous and Discrete Time Problems", working paper

Chou Chien-fu, and Gabriel Talmain (1996); "Redistribution and Growth: Pareto Improvements", Journal of Economic Growth, Vol. 1, Issue 4, pp. 505- 523.

Chu, Angus C. (2010); "Effects of Patent Policy on Income and Consumption Inequality in an R\&D-Growth Model", Southern Economic Journal, Vol. 77, No. 2, pp. 336-350

Chu, Angus C. and Guido Cozzi (2016); "Patents vs R\&D Subsidies on Income Inequality", MPRA working paper No. 73482, November 2016

Chu, Angus C. and Shin-Kun Peng (2011); "International intellectual property rights: Effects on growth, welfare and income inequality", Journal of Macroeconomics, Volume 33, Issue 2, pp. 276-287

Chung, Kim-Sau and Chia-Hui Lu (2014); "Non-homothetic preferences and IPRs protection", Journal of Development Economics, Vol. 109, pp. 229-239

Cozzi, Guido \& Silvia Galli (2014); "Sequential R\&D and blocking patents in the dynamics of growth", Journal of Economic Growth, Springer, vol. 19(2), pp. 183-219

Cysne, R. and D. Turchick (2012); "Intellectual property rights protection and endogenous economic growth revisited", Journal of Economic Dynamics and Control, 36, pp. 851-861

Deneckere, R. and K. Judd (1992); "Cyclical and chaotic behavior in a dynamic equilibrium model", in "Cycles and Chaos in Economic Equilibrium", ed. by J. Benhabib, Princeton: Princeton Univ. Press

Dorn, Sabrina and Christian Kiedaisch (2017); "Inequality and Demand-Driven Innovation: Evidence from International Patent Applications", mimeo, University of Zurich

Falkinger, Josef (1994); "An Engelian Model of Growth and Innovation with Hierarchic Demand and Unequal Incomes", Ricerche Economiche 48, 123-139.

Falkinger, Josef and Josef Zweimüller (1996); "The Cross-Country Engel-Curve for Product Diversification", Structural Change and Economic Dynamics, 7.

Föllmi, Reto, Würgler, Tobias and Josef Zweimüller (2014); "The Macroeconomics of Model T" Journal of Economic Theory 153, pp. 617- 647. 
Föllmi, Reto and Josef Zweimüller; (2002); „Structural Change and the Kaldor Facts of Economic Growth", IZA discussion paper.

Föllmi, Reto and Josef Zweimüller (2006); "Income distribution and demandinduced innovations", Review of Economic Studies 73(4), pp. 941-960

Föllmi, Reto and Josef Zweimüller (2016); "Is Inequality harmful for Innovation and Growth: Price versus Market Size Effects", Journal of Evolutionary Economics, 26(1), pp. $1-20$

Furukawa, Yuichi (2007); "The protection of intellectual property rights and endogenous growth: Is stronger always better?", Journal of Economic Dynamics \& Control 31, pp. 3644-3670

Gilbert, R. and C. Shapiro (1990); "Optimal Patent Length and Breadth", RAND Journal of Economics 21: pp. 106-12

Grossman, G. M. and E. Helpman (1992); "Innovation and Growth in the global Economy", (Cambridge MA: MIT Press).

Hatipoglu, Ozan (2012); "The relationship between inequality and innovative activity: a Schumpeterian theory and evidence from cross-country data", Scottish Journal of Political Economy, Vol. 59, No. 2

Jaravel, Xavier (2017); "The Unequal Gains from Product Innovations: Evidence from the US Retail Sector", working paper

Jackson L. F. (1984); "Hierarchic Demand and the Engel Curve for Variety", Review of Economics and Statistics, 66 (1).

Judd, Kenneth L. (1985); "On the Performance of Patents", Econometrica Vol. 53, No. 3, pp. 567-586

Kiedaisch (2009); "Patent Policy and Income Distribution", working paper, ETH Zurich

Kiedaisch (2009); "Varying Patent Strength and the Allocation of R\&D across Sectors", working paper, ETH Zurich

Kohler, Andreas (2012); "Trade and Growth in an Unequal Global Economy", University of Zurich Working Paper No. 81

Kwan, Y. K. and E. Lai (2003); "Intellectual Property Rights Protection and Endogenous Economic Growth", Journal of Economic Dynamics and Control, 27 (5), 853-873

Llanes, G. and S. Trento (2012); "Patent policy, patent pools, and the accumulation of claims in sequential innovation", Economic Theory, 50 (3), pp. 703-725

Matsuyama, Kiminori (1999); "Growing through Cycles", Econometrica, Vol. 67, No. 2, pp. 335-347

Matsuyama, Kiminori (2002); "The rise of Mass Consumption Societies", Journal of Political Economy, Vol. 110

Michel, P. and J. Nyssen (1998); "On Knowledge Diffusion, Patents Lifetime and Innovation Based Endogenous Growth", Annales d'Economie et de Statistiques, vol. 49/50, pp.79-103.

Murphy, Kevin M., Andrei Shleifer, and Robert W. Vishny (1989); "Income Distribution, Market Size, and Industrialization", Quarterly Journal of Economics 104, 537-564.

O' Donoghue, T. and J. Zweimüller (2004); "Patents in a model of endogenous growth", Journal of Economic Growth, 9 (1), pp. 81-123

Pan, Shiyuan, Zou, Heng-fu and Tailong Li (2015?); "Patent Protection, Technological Change and Wage Inequality", working paper 
Piketty, T. (2014); "Capital in the Twenty-First Century", Cambridge MA: The Belknap Press of Harvard University Press.

Rivera-Batiz, L. and P. Romer (1991); "Economic integration and endogenous growth", Quarterly Journal of Economics, 106 (1991), pp. 531-555

Romer, Paul M. (1990); "Endogenous Technological Change", Journal of Political Economy, 98 (5); pp. S71- S102

Saggi, Kamal (2016); "Trade, Intellectual Property Rights, and the World Trade Organization", The Handbook of Commercial Policy, Volume 1, pp. 433-512, NorthHolland

Saini, Swati and Meeta Mehra (2014); "Impact of strengthening Intellectual Property Rights Regime on income inequality: An econometric analysis," MPRA Paper 56710, University Library of Munich,

Saint-Paul, Gilles (2004); "Are Intellectual Property Rights Unfair?", Labour Economics, vol. 11, n. 1, p. 129-144.

Saint-Paul, Gilles (2006); "Distribution and Growth in an Economy with Limited Needs: Variable Markups and the 'End of Work'", The Economic Journal vol. 116, n. 511, pp. 382-407

Spinesi, Luca (2011); "Probabilistic Heterogeneous Patent Protection and Innovation Incentives", The B.E. Journal of Economic Analysis \& Policy, Vol. 11, Iss. 1 (Contributions), Article 45.

Würgler, Tobias (2010); "Income Distribution and Product Quality versus Variety", working paper, University of Zürich

Zweimüller, Josef (2000); "Schumpeterian Entrepreneurs meet Engel's Law: The Impact of Inequality on Innovation-Driven Growth", Journal of Economic Growth, 5, pp. $185-206$ 


\section{Graphs}

Figure 2: Market demand of a monopolistic firm

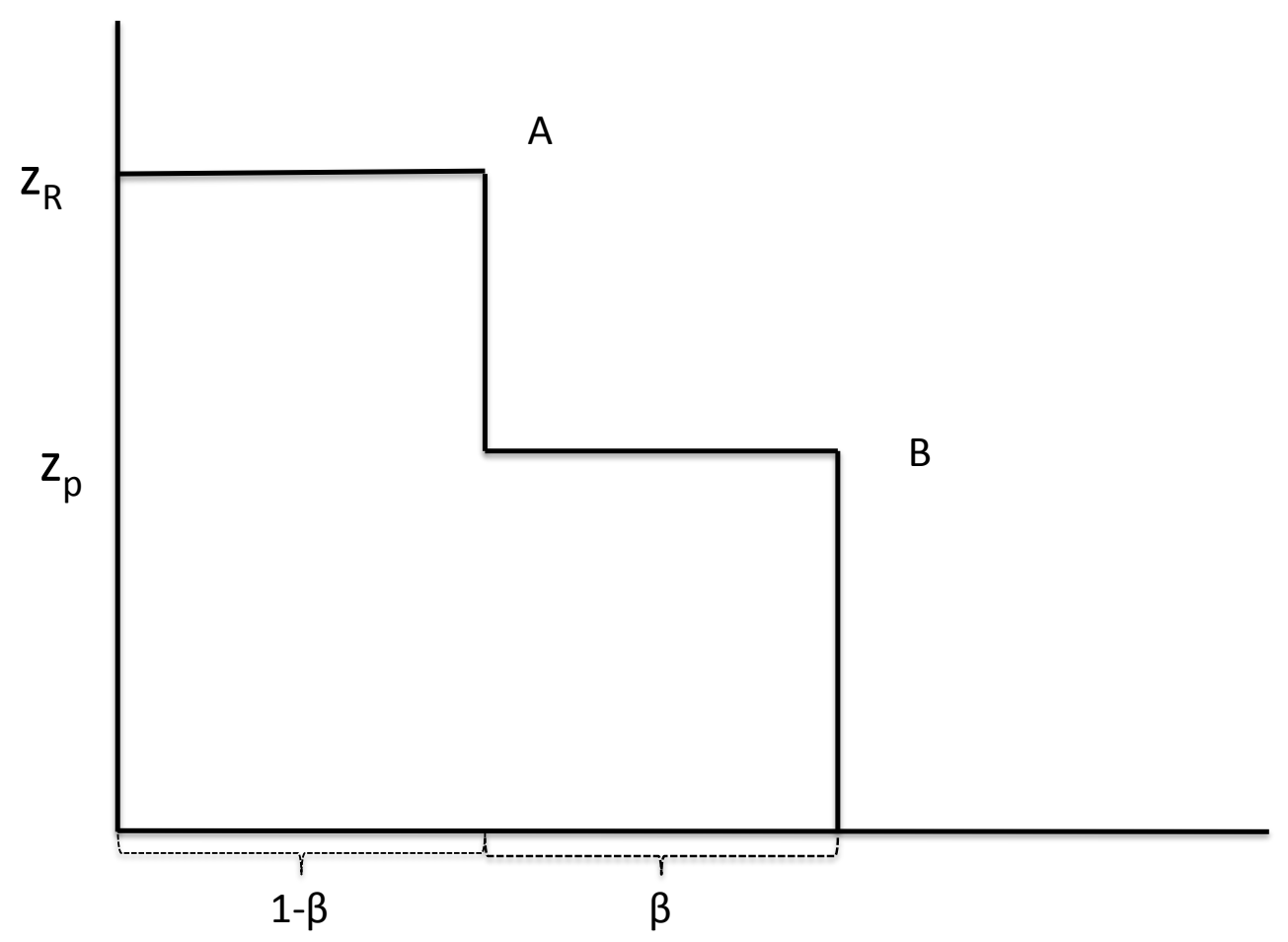

Figure 3: Price structure and consumption in Regime A

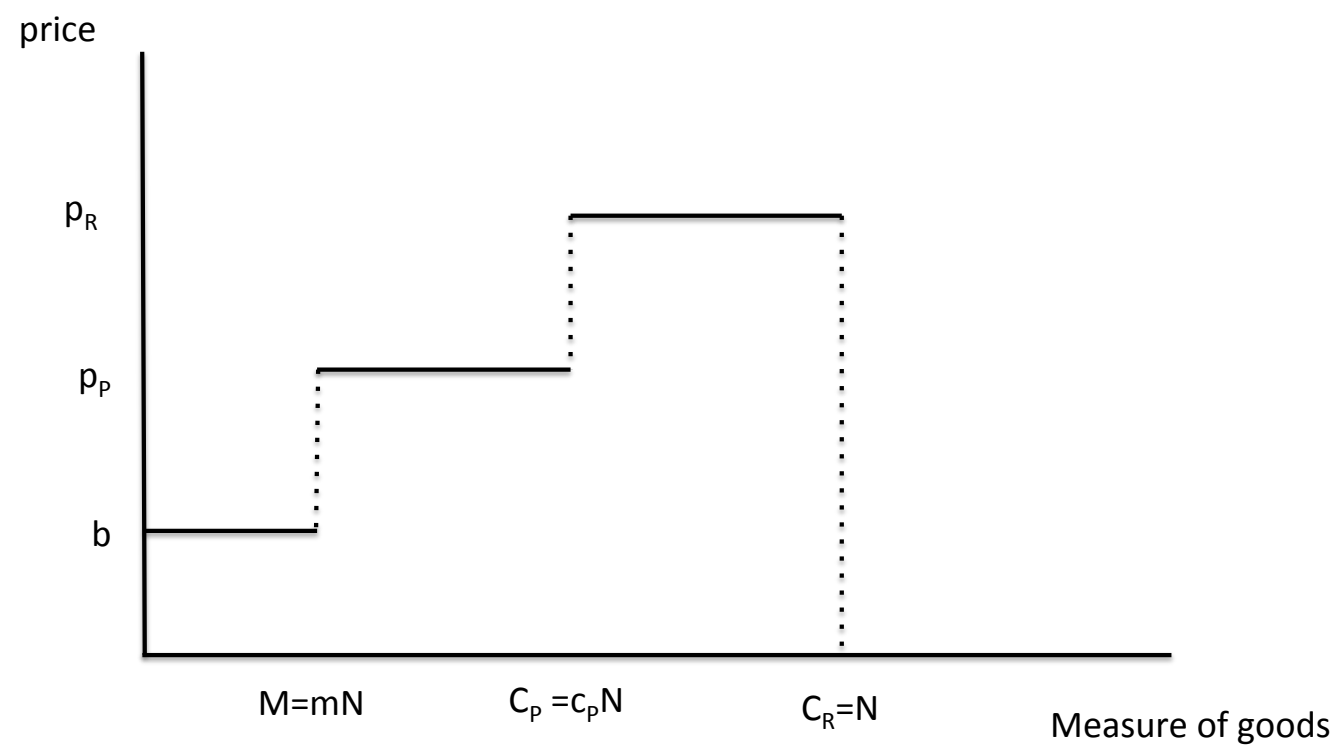


Figure 4: The general equilibrium

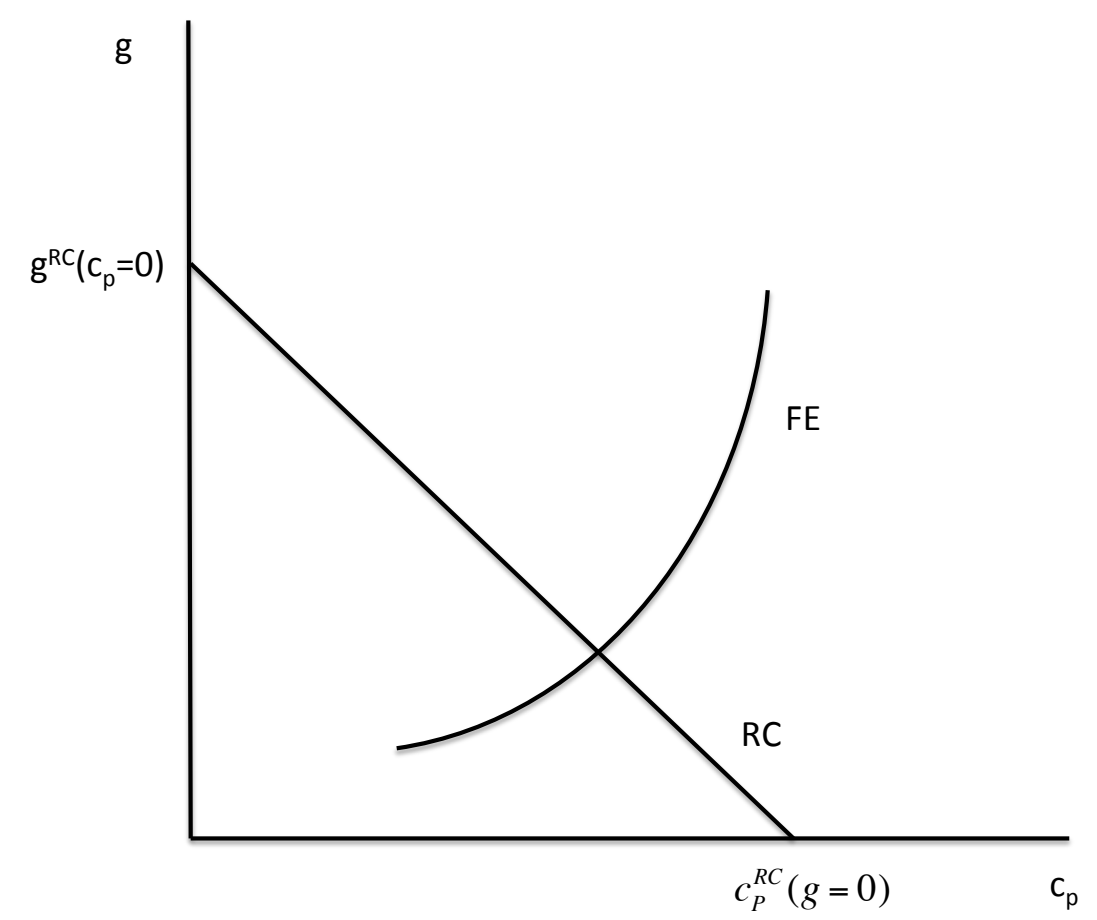

Figure 5: Price structure and consumption in Regime B

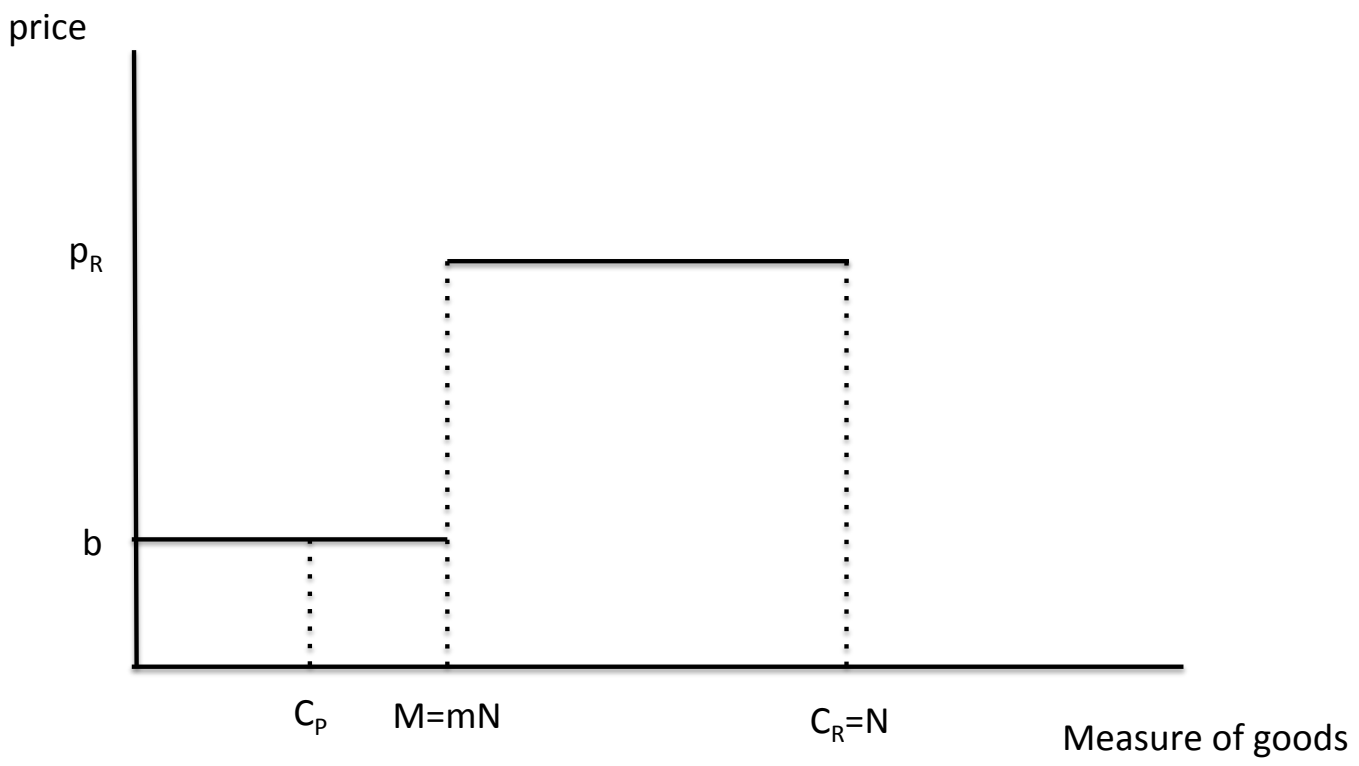


Figure 6: Price structure and consumption in Regime C1

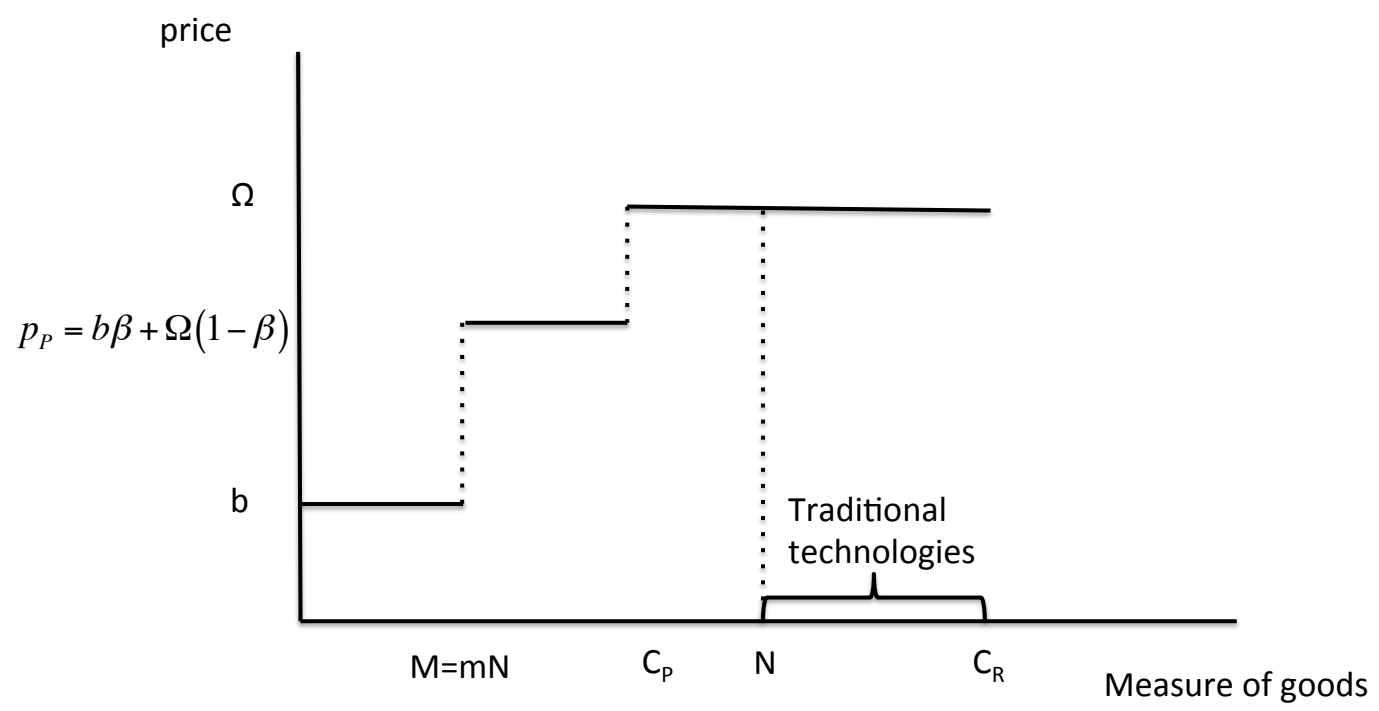

Figure 7: Price structure and consumption in Regime C2

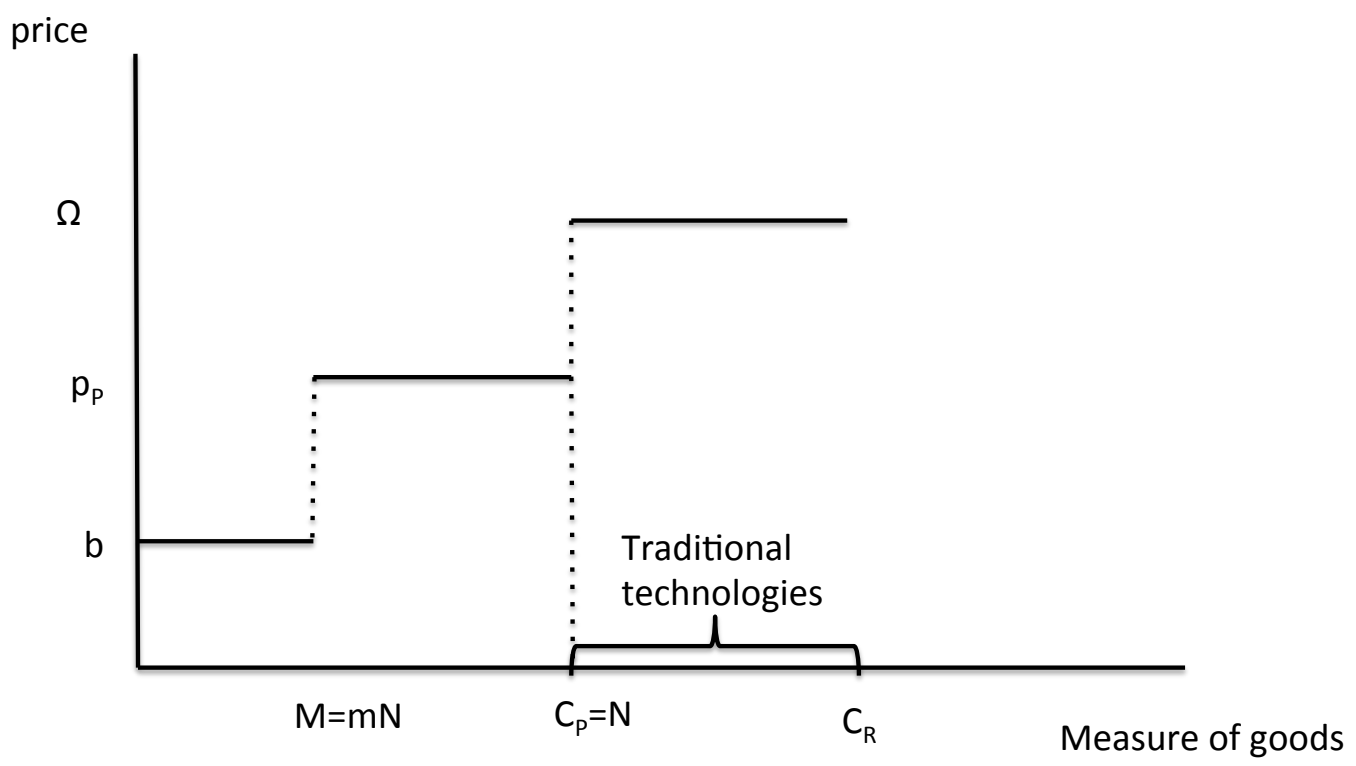


Figure 8: Price structure and consumption in Regime C3

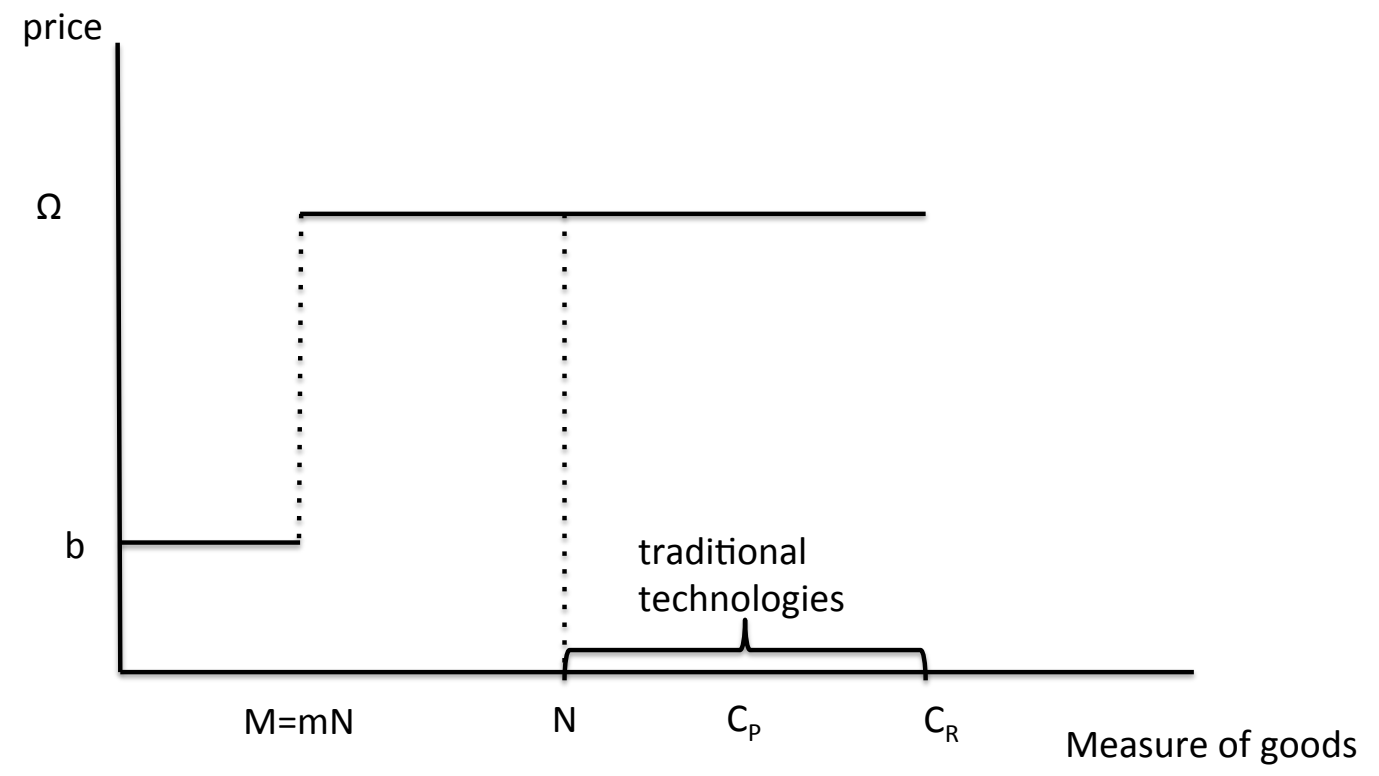




\section{For online publication: Appendix A}

\section{A1 Proof of Proposition 1}

Proof. In the following, the shapes of the free entry (FE, equation 16) and the resource constraint (RC, equation 5) curves are analyzed in $g-c_{P}$ space (with the latter variable on the horizontal axis) and the comparative static results are derived. A graphical representation is given in Figure 4.

The RC curve is continuous, linear and downward sloping if $b>0$ (Condition $\mathbf{C}$ ) and crosses the $g$ - axis at $g^{R C}\left(c_{P}=0\right)=\frac{1-b(1-\beta)}{F}$ and the $c_{P}$ - axis at $c_{P}^{R C}(g=0)=$ $\frac{1-b(1-\beta)}{b \beta} 57 . \quad b<\frac{1}{1-\beta}$ (Condition $\mathbf{C}$ ) therefore needs to hold in order to ensure that $g>0$ and $c_{P}>0$ can hold. For $c_{P}<1$, the FE curve is continuous and upward sloping. It crosses the $c_{P}$ - axis at $c_{P}^{F E}(g=0)=1-\frac{1-\vartheta+(1-\beta) \rho X}{F(\rho+\gamma)+(1-\beta) b}<1$ and the $g$ axis at $g^{F E}\left(c_{P}=0\right)=\left(\frac{1-\vartheta+(1-\beta)(\rho X-b)}{F}\right)-\rho-\gamma$ and approaches the asymptote $c_{P}=1$ $\left(\lim _{g \rightarrow \infty} c_{P}^{F E}=1\right)$. Therefore, $c_{P}<1$ always holds. Given Condition $\mathrm{C}$ holds, the two curves cross in the positive quadrant if $g^{F E}\left(c_{P}=0\right)<g^{R C}\left(c_{P}=0\right)$ (Condition D) and if $c_{P}^{F E}(g=0)<c_{P}^{R C}(g=0)$ (Condition $\mathbf{E}$ ). These conditions are satisfied if $\vartheta>$ $(1-\beta) \rho X-F(\rho+\gamma)(\mathbf{D})$ and if $\vartheta<(1-\beta) \rho X+\frac{F(\rho+\gamma)(1-b)}{b \beta}+\frac{1-b}{\beta}+b(\mathbf{E})$ hold. In order to ensure that $V_{P}(t)=(1-m) F N(t)-(1-\beta) X N(t) \geq 0$ (see equation 14), $X \leq \tilde{X} \equiv \frac{(1-m) F}{1-\beta}$ (Condition B) needs to be satisfied, where $\tilde{X}>0$ as $m=\frac{\gamma}{g+\gamma}<1$ must hold along any BGP with positive growth. In order to obtain a BGP in Regime A, Condition A $\left(c_{P}>m\right)$ has to hold. For any fixed value of $g>0, m=\frac{\gamma}{g+\gamma}$ continuously increases from 0 to 1 if $\gamma$ increases from 0 to $\infty$. As Condition $\mathrm{D}$ implies that $c_{P}>0$ holds (even if $\gamma=0$ ), there is always a positive threshold $\tilde{\gamma}$ (or a finite threshold $\tilde{T}=\frac{1}{\tilde{\gamma}}$ ), such that for $0 \leq \gamma<\tilde{\gamma}$ ( or $\tilde{T}<T \leq \infty$; Condition $\mathbf{F}$ ), $c_{P}>m$ holds along the BGP.

The comparative static results a), b), and c) can be derived by analyzing how a change in an exogenous parameter shifts the RC and the FE curves: an increase in $\gamma$ (i.e. a reduction in $T$ ) or $\vartheta$ and a decrease in $X$ shift the FE curve downwards and leave the $\mathrm{RC}$ unaffected, implying a reduction in $g$ and an increase in $c_{P}$. An increase in $F$ and a reduction in $\beta$ shift both the $\mathrm{RC}$ and the $\mathrm{FE}$ curves downward and lead to a reduction in $g$. Deriving the FE condition (16) with respect to $\rho$ gives $\left.\frac{\partial g}{\partial \rho}\right|_{F E}=\frac{X(1-\beta)}{F\left(1-c_{P}\right)}-1$. Therefore, $\left.\frac{\partial g}{\partial \rho}\right|_{F E}>0$ holds if $X>\frac{\left(1-c_{P}\right) F}{1-\beta} \equiv \hat{X}>0$. This condition is compatible with Condition $\mathrm{B}\left(X \leq \frac{(1-m) F}{1-\beta} \equiv \tilde{X}\right)$ due to Condition $\mathrm{A}\left(c_{P}>m\right)$. Therefore, an increase in $\rho$ shifts the FE curve upward if $\hat{X}<X \leq \tilde{X}$, while it shifts it downward if $0 \leq X<\hat{X}$. As the RC curve does not depend on $X$, the BGP growth rate $g$ therefore increases in $\rho$ if $\hat{X}<X \leq \tilde{X}$ and decreases in $\rho$ if $0 \leq X<\hat{X}$.

\footnotetext{
${ }^{57}$ The notation $x^{y}(z=k)$ indicates the value $x$ given by equation (curve) $y$ if $z$ has value $k$.
} 


\section{A2 Proof of Proposition 2}

Proof. In the following it is shown that all equilibrium conditions are satisfied if $g$ and $c_{P}, r, p_{R}$ and $p_{P}$ immediately jump to their new BGP values and if $X$ immediately jumps from $X_{0}$ to $X_{1}$ when an unexpected change in IP policy or in one of the other exogenous parameters occurs in $t=t_{0}$. The following notation is used: old BGP values are labeled with the subscript ${ }_{o}$ while new BGP values are not labeled.

As neither the resource constraint (equation 5) nor the free entry condition (equation 16 with $X$ replaced by $X_{1}=\frac{\rho+g+\gamma_{1}}{\rho+g+\gamma_{0}} X_{o}$ ) directly depends on the sluggishly adjusting state variables $V$ and $m$, it suffices to show that the intertemporal budget constraints, in which both $V$ and $m$ enter, are still fulfilled with equality if $g, c_{P}, r, p_{R}, p_{P}$, and $X$ immediately jump to their new BGP values while $m$ and $V$ adjust slowly. As $p_{R}$ (equation 15) is derived by subtracting the budget constraint of a poor household from that of a rich household, the latter is always satisfied if $p_{R}$ is constant, so that it suffices to check only whether the budget constraint of a poor household is satisfied with equality.

At point in time $t=t_{0}$, the number of competitively supplied goods is given by the old BGP value $M\left(t_{0}\right)=m_{o} N\left(t_{0}\right)$. When prices immediately jump to their new BGP value in $t_{0}, p_{R}$ can be derived as

$$
p_{R}=\frac{F\left(\rho+g+\gamma_{1}\right)}{1-\beta}+b
$$

from the free entry condition (equation 7) that is forward looking and satisfied at each instant of time. Taking into account that the stock of existing IPRs expires at rate $\gamma_{0}$ after $t_{0}$, so that the value of one of these IPRs is given by $Z_{o}=\frac{(1-\beta)\left(p_{R}-b\right)}{\rho+g+\gamma_{0}}=F\left(\frac{\rho+g+\gamma_{1}}{\rho+g+\gamma_{0}}\right)$, the total value of initial wealth at point in time $t=t_{0}$ is given by

$$
V\left(t_{0}\right)=N\left(t_{0}\right)\left(1-m_{o}\right) Z_{o}=N\left(t_{0}\right)\left(1-m_{o}\right) F\left(\frac{\rho+g+\gamma_{1}}{\rho+g+\gamma_{0}}\right)
$$

If $\gamma_{1}=\gamma_{2}$, the value of initial wealth therefore does not change in $t_{0}$, even if the length of IP protection is changed (i.e. if $\gamma \neq \gamma_{1}=\gamma_{2}$ ). The initial wealth of a poor household can be (like in equation 14) derived as

$$
V_{P}\left(t_{0}\right)=N\left(t_{0}\right)\left(\frac{\rho+g+\gamma_{1}}{\rho+g+\gamma_{0}}\right)\left[\left(1-m_{o}\right) F-(1-\beta) X_{o}\right]
$$

Plugging this expression and equation 4 into equation 9 and replacing $\frac{N\left(t_{0}\right) m}{r-g}$ with $\int_{t=t_{0}}^{\infty} M(t) e^{-(\rho+g)\left(t-t_{0}\right)} d t$ to account for the fact that $m(t)$ changes during the transition, the intertemporal budget constraint of a poor household at point in time $t_{0}$ can be written 


$$
\begin{aligned}
\frac{N\left(t_{0}\right)}{p_{P}-b}\left[\frac{p_{P} c_{P}-\vartheta}{\rho}+\left(\frac{\rho+g+\gamma_{1}}{\rho+g+\gamma_{0}}\right)\left[(1-\beta) X_{o}-(1-\right.\right. & \left.\left.\left.m_{o}\right) F\right]\right] \\
& =\int_{t=t_{0}}^{\infty} M(t) e^{-(\rho+g)\left(t-t_{0}\right)} d t
\end{aligned}
$$

In order to replace the integral on the right hand side, $M(t)$ has to be determined.

Defining the stocks of IPRs granted before and after $t_{0}$ by $P^{0}(t)$ and $P^{1}(t)$, the differential equation

$$
\frac{\partial M(t)}{\partial t}=\gamma_{0} P^{0}(t)+\gamma_{1} P^{1}(t)
$$

determines the evolution of $M(t)$ for $t>t_{0}$ as the measure $M(t)$ of competitively supplied goods increases due to the expiration of both stocks of IPRs. As $\frac{\partial P^{0}(t)}{\partial t}=-\gamma_{0} P^{0}(t)$ and as $P^{0}\left(t_{0}\right)=N\left(t_{0}\right)\left(1-m_{o}\right), P^{0}(t)$ is given by

$$
P^{0}(t)=N\left(t_{0}\right)\left(1-m_{o}\right) e^{-\gamma_{0}\left(t-t_{0}\right)}
$$

From the differential equation $\frac{\partial P^{1}(t)}{\partial t}=\frac{\partial N(t)}{\partial t}-\gamma_{1} P^{1}(t)=g N(t)-\gamma_{1} P^{1}(t)$ and the initial condition $P^{1}\left(t_{0}\right)=0$, the evolution of the stock $P^{1}(t)$ of IPRs granted after $t_{0}$ can be derived as

$$
P^{1}(t)=\frac{g N\left(t_{0}\right)}{g+\gamma_{1}}\left[e^{g\left(t-t_{0}\right)}-e^{-\gamma_{1}\left(t-t_{0}\right)}\right]
$$

Therefore,

$\frac{\partial M(t)}{\partial t}=\gamma_{0} P^{0}(t)+\gamma_{1} P^{1}(t)=\gamma_{0} N\left(t_{0}\right)\left(1-m_{o}\right) e^{-\gamma_{0}\left(t-t_{0}\right)}+\gamma_{1} \frac{g N\left(t_{0}\right)}{g+\gamma_{1}}\left[e^{g\left(t-t_{0}\right)}-e^{-\gamma_{1}\left(t-t_{0}\right)}\right]$

Taking into account that $M\left(t_{0}\right)=N\left(t_{0}\right) m_{o}$, we can derive

$$
M(t)=N\left(t_{0}\right)\left[\frac{\gamma_{1}}{g+\gamma_{1}} e^{g\left(t-t_{0}\right)}+\frac{g}{g+\gamma_{1}} e^{-\gamma_{1}\left(t-t_{0}\right)}-\left(1-m_{o}\right) e^{-\gamma_{0}\left(t-t_{0}\right)}\right]
$$

so that

$$
\int_{t=t_{0}}^{\infty} M(t) e^{-(\rho+g)\left(t-t_{0}\right)} d t=N\left(t_{0}\right)\left[\frac{\gamma_{1}}{\left(g+\gamma_{1}\right) \rho}+\frac{g}{\left(g+\gamma_{1}\right)\left(\rho+g+\gamma_{1}\right)}-\frac{1-m_{o}}{\rho+g+\gamma_{0}}\right]
$$

Plugging this, $p_{P}=F\left(\rho+g+\gamma_{1}\right)+b$ (derived from equations 4 and 7 with $\gamma$ replaced by $\left.\gamma_{1}\right)$ and $c_{P}=\frac{1}{b \beta}(1-g F-b(1-\beta)$ ) (from equation 5$)$ into equation 19 gives the following cubic equation that determines $g$

$$
\begin{gathered}
-g^{3} F^{2}+g^{2} F\left[1+b \beta-2 b-F\left(2 \rho+\gamma_{0}+\gamma_{1}\right)\right]+g b\left[1-\vartheta \beta-b(1-\beta)+\beta(1-\beta) X_{o} \rho\right] \\
+g F\left[2 \rho+\gamma_{0}+\gamma_{1}-F\left(\rho+\gamma_{0}\right)\left(\rho+\gamma_{1}\right)-b\left(\rho(3-\beta)+\gamma_{0}(2-\beta)+\gamma_{1}\right)\right]+
\end{gathered}
$$




$$
\left(\rho+\gamma_{0}\right)\left[F\left(\rho+\gamma_{1}\right)(1-b)-b(\vartheta \beta-1+b(1-\beta))\right]+b \beta(1-\beta) X_{o} \rho\left(\rho+\gamma_{1}\right)=0
$$

This equation is independent of $m_{o}$ and the same as that that results when the new BGP value of $g$ is determined by plugging the free entry condition (equation 16, with $X_{o}$ replaced by $X_{1}=\frac{\rho+g+\gamma_{1}}{\rho+g+\gamma_{0}} X_{o}$ ) into the resource constraint (equation 5). Therefore, the intertemporal budget constraint of a poor household is satisfied with equality if $g, c_{P}$, $r, p_{R}, p_{P}$ and $X$ immediate jump to the new BGP values in $t_{0}$ while $m$ (and $V$ ) adjust sluggishly.

\section{A3 Proof of Corollary 1}

Proof. While the resource constraint is always given by equation 5 , the free entry condition is given by

$$
g^{o}=\left(\frac{\frac{1-\vartheta}{1-\beta}+\rho X_{o}}{1-c_{P}^{o}}-b\right) \frac{1-\beta}{F}-\rho
$$

before $t_{0}$ and by

$$
g=\left(\frac{\frac{1-\vartheta}{1-\beta}+\rho\left(\frac{\rho+g+\gamma_{1}}{\rho+g}\right) X_{o}}{1-c_{P}}-b\right) \frac{1-\beta}{F}-\rho-\gamma_{1}
$$

after $t_{0}$ (see equation 16 and Proposition $2 \mathrm{~b}$ with $\gamma_{0}=0$ ). The new BGP value $g$ therefore lies above the old one $g^{o}$ if the new free entry curve lies above the old one (i.e. if for given value of $c_{p}$, the value of $g$ is larger in the new FE condition). This is the case if

$$
X_{0}>\frac{F\left(1-c_{P}\right)(\rho+g)}{(1-\beta) \rho}
$$

holds for both the old and the new BGP values of $g$ and $c_{P}$. Plugging in the maximal value $X_{o}=\frac{F}{1-\beta}$ for which $V_{P}^{o}=0$ (see equation 14 with $m=0$; this coincides with the case where $X=\widetilde{X}$ in Proposition 1, implying that Condition B is satisfied with equality), this condition becomes

$$
c_{P}>1-\frac{\rho}{\rho+g}
$$

(Condition G). As only a marginal increase in $\gamma_{1}$ is analyzed and as the new FE condition coincides with the old one for $\gamma_{1}=0$, it suffices to show that Condition $\mathrm{G}$ is satisfied for the old BGP values $g^{o}$ and $c_{p}^{o}$. Plugging the resource constraint (equation 5) into the free entry condition gives a quadratic equation with the interior solution

$$
c_{P}^{o}=\frac{1+b \beta+F \rho-\sqrt{(1+b \beta+F \rho)^{2}-4 b \beta \vartheta}}{2 b \beta}
$$


Plugging this into the resource constraint gives

$$
g^{o}=\frac{1}{2 F}\left[1+b \beta-2 b-F \rho+\sqrt{(1+b \beta+F \rho)^{2}-4 b \beta \vartheta}\right]
$$

Plugging these values into Condition $G$ leads to the inequality

$$
\sqrt{(1+b \beta+F \rho)^{2}-4 b \beta \vartheta}\left(\frac{1}{\beta}-1\right)-\left[\frac{1+b \beta+F \rho}{\beta}+1+b \beta-2 b-\rho F-2 \vartheta\right]>0
$$

If $\vartheta=1, b=\frac{1}{2}$ and $F=\frac{\beta}{2 \rho}$, this inequality is satisfied and an interior solution $\left(0<c_{P}^{o}<1\right.$ and $\left.g^{o}>0\right)$ is obtained. Therefore, $g$ increases in $t_{0}$ in this case $\mathrm{e}^{58}$.

\section{A4: Proof of Proposition 3}

Proof. a): As the prices of the competitively supplied goods are equal to the marginal cost $b$, the expenditures $E_{P}(t)$ of a poor household that only purchases these kind of goods are equal to $Y_{P}(t)=b C_{P}(t)$ in period $t$. Along a BGP on which $C_{P}(t)\left(=c_{P}(t) N(t)\right)$ and the labor income $\vartheta N(t)$ of a poor household grow at rate $g$, the intertemporal budget constraint of such a household can (like in equation 9) be derived as

$$
\frac{N(\tau) \vartheta}{r-g}+V_{P}(\tau)=\frac{N(\tau)}{r-g} c_{P} b
$$

Taking into consideration that $r=\rho+g$ (equation 6) holds along a BGP and using the definition $\bar{V}_{P} \equiv \frac{V_{P}(\tau)}{N(\tau)}$ gives

$$
c_{P}=\frac{\vartheta+\rho \bar{V}_{P}}{b}
$$

In order to obtain an interior solution $c_{P}<1, \vartheta+\rho \bar{V}_{P}<b$ has to hold. Plugging equation 21 into the resource constraint (equation 5) allows the derivation of the BGP growth rate as

$$
g=\frac{1}{F}\left[1-b(1-\beta)-\beta\left(\vartheta+\rho \bar{V}_{P}\right)\right]
$$

In order for a BGP with $g>0$ to exist, $1-b(1-\beta)-\beta\left(\vartheta+\rho \bar{V}_{P}\right)>0$ therefore has to hold. Moreover, $c_{P}<m$ needs to hold in Regime B (Condition A must be violated). Plugging equation 21 and $m=\frac{\gamma}{g+\gamma}$ (which can again be derived like in equation 8) with

\footnotetext{
${ }^{58}$ During the transition period, $m$ increases and the normalized value of wealth $\frac{V(t)}{N(t)}$ decreases. As $X$ is constant during the transition (see the proof of Proposition 2), this implies that the value of initial wealth of a poor household, $V_{P}(t)=V(t)-(1-\beta) X N(t)$, falls below zero in the long run because it is equal to zero at $t_{0}$. In the example analyzed in Corollary 1 , poor households therefore end up with a small amount of debt in the long run $\left(V_{P}<0\right)$, a feature that was excluded by assumption in the previous analysis. The qualitative result of Corollary 1 does, however, not depend on this feature: As the relation between $X, g$ and $c_{P}$ is continuous and as only a marginal reduction in the length of IP protection is analyzed ( $\gamma_{1}$ is small), the qualitative result of Corollary 1 can still be obtained in cases where poor households start with a slightly positive amount of initial wealth (i.e. if $X_{o}=\frac{F}{1-\beta}-\epsilon$ with $\epsilon$ small) and never accumulate debt so that $V_{P}>0$ always holds.
} 
$g$ replaced by its BGP value into this condition and solving the resulting inequality for $\gamma$ gives the inequality $\gamma>\frac{\left(\vartheta+\rho \bar{V}_{P}\right)\left(1-b(1-\beta)-\beta\left(\vartheta+\rho \bar{V}_{P}\right)\right)}{F\left(b-\vartheta-\rho \bar{V}_{P}\right)}$ under which Condition A is violated. Given that $g>0$ and $c_{P}<1$, both the numerator and the denominator of the right hand side of this inequality are positive, so that $\gamma>0$ has to hold. Condition A is consequently violated if $T \equiv \frac{1}{\gamma}<\tilde{T}=\frac{F\left(b-\vartheta-\rho \bar{V}_{P}\right)}{\left(\vartheta+\rho \bar{V}_{P}\right)\left(1-b(1-\beta)-\beta\left(\vartheta+\rho \bar{V}_{P}\right)\right)}<\infty$ holds.

b) and c): As in the proof of Proposition 2 it is now shown that all equilibrium conditions are satisfied if $g, c_{P}, r$ and $p_{R}$ immediately jump to their new BGP values and if $\bar{V}_{P}$ immediately jumps from $\bar{V}_{P}^{o}$ to $\bar{V}_{P}^{1}$ when an unexpected change in IP policy or in one of the other exogenous parameters occurs in $t=t_{0}$. Old BGP values are again labeled with the subscript ${ }_{o}$ while new BGP values are not labeled.

As poor households do not consume IP protected goods and are therefore not confronted with changing consumption prices, they spend all their labor income $\vartheta N(t)$ and in addition the amount $(r-g) \bar{V}_{P} N(t)=\rho \bar{V}_{P} N(t)$ every period, implying that $V_{P}(t)$ grows at rate $g$ and that $\bar{V}_{P}$ is constant after $t_{0}$. What remains to be shown is that the intertemporal budget constraint of a rich household is satisfied with equality if the transition of $m$ is taken into account.

When prices immediately jump to their new BGP value in $t_{0}$, equation 7 can be used to derive $p_{R}$ (the price at which IP protected goods are sold to rich households) as:

$$
p_{R}=\frac{F\left(\rho+g+\gamma_{1}\right)}{1-\beta}+b
$$

As the stock of existing IPRs expires at rate $\gamma_{0}$ after $t_{0}$, so that the value of one of these IPRs is given by $Z_{o}=\frac{(1-\beta)\left(p_{R}-b\right)}{\rho+g+\gamma_{0}}=F\left(\frac{\rho+g+\gamma_{1}}{\rho+g+\gamma_{0}}\right)$, the total value of initial wealth at point in time $t=t_{0}$ is given by

$$
V\left(t_{0}\right)=N\left(t_{0}\right)\left(1-m_{o}\right) Z_{o}=N\left(t_{0}\right)\left(1-m_{o}\right) F\left(\frac{\rho+g+\gamma_{1}}{\rho+g+\gamma_{0}}\right)
$$

The initial wealth of a poor household therefore changes from $\bar{V}_{P}^{o}$ to $\bar{V}_{P}^{1}=\left(\frac{\rho+g+\gamma_{1}}{\rho+g+\gamma_{0}}\right) \bar{V}_{P}^{o}$ in $t_{0}$. As $V=\beta V_{P}+(1-\beta) V_{R}$ and as $\bar{V}_{P}^{o}=\frac{V_{P}^{o}}{N}$, the stock of initial wealth held by a rich household in $t_{0}$ can be derived as

$$
V_{R}\left(t_{0}\right)=\frac{N\left(t_{0}\right)}{1-\beta}\left[\frac{\rho+g+\gamma_{1}}{\rho+g+\gamma_{0}}\right]\left(\left(1-m_{o}\right) F-\beta \bar{V}_{P}^{o}\right)
$$

The intertemporal budget constraint of a rich household is given by

$$
\frac{N\left(t_{0}\right)}{(r-g)} \frac{(1-\beta \vartheta)}{(1-\beta)}+V_{R}\left(t_{0}\right)=\int_{t=t_{0}}^{\infty}\left[M(t) b+(N(t)-M(t)) p_{R}\right] e^{-r\left(t-t_{0}\right)} d t
$$


as rich households buy the measure $M(t)$ of competitively supplied goods sold at price $b$ and the measure $N(t)-M(t)$ of IP protected goods sold at price $p_{R}$ in period $t$. Replacing $r, p_{R}$, and $V_{R}\left(t_{0}\right)$, this budget constraint can be written as

$$
N\left(t_{0}\right)\left[\frac{1}{\rho}-\frac{1-\beta \vartheta-b(1-\beta)}{F \rho\left(\rho+g+\gamma_{1}\right)}-\frac{\left(1-m_{o}\right) F-\beta \bar{V}_{P}^{o}}{F\left(\rho+g+\gamma_{0}\right)}\right]=\int_{t=t_{0}}^{\infty} M(t) e^{-r\left(t-t_{0}\right)} d t
$$

Replacing the right hand side by equation 20 that has been derived in the proof of Proposition 2, the budget constraint can be rewritten as:

$$
g=\frac{1}{F}\left[1-b(1-\beta)-\beta \vartheta-\beta \rho \bar{V}_{P}^{o}\left(\frac{\rho+g+\gamma_{1}}{\rho+g+\gamma_{0}}\right)\right]
$$

As this is the same equation as equation 22 with $\bar{V}_{P}$ replaced by $\bar{V}_{P}^{1}=\bar{V}_{P}^{o}\left(\frac{\rho+g+\gamma_{1}}{\rho+g+\gamma_{0}}\right)$, the intertemporal budget constraints of rich households are satisfied with equality when $g$, $c_{P}, p_{R}, r$ and $\bar{V}_{P}$ immediately jump to their new BGP values while $m$ and $\frac{V_{R}(t)}{N(t)}$ adjust sluggishly.

\section{A5: Proof of Proposition 4}

Proof. a) Rich households benefit from any policy that increases $g$ as $U_{R}(\tau)$ increases in $g$ (see equation 17). Plugging equation 5 (which holds in both regimes) into equation 18 gives $U_{P}(\tau)$ as a continuous and concave function of $c_{P}$. As $U_{P}(\tau)$ is maximal for $c_{P}=\frac{F \rho}{b \beta}$, poor households benefit from policies that reduce $c_{P}$ and therefore (taking equation 5 into account) increase $g$ if $c_{P}>\frac{F \rho}{b \beta}$ and are harmed by them if $c_{P}<\frac{F \rho}{b \beta}$. As $c_{P}<1$, poor households therefore always want to reduce $g$ if $\frac{F \rho}{b \beta}>1$ (there is a non-empty range of parameters for which this condition is compatible with the existence of a BGP in both Regime A and B).

In Regime $\mathrm{B}, c_{P}=\frac{\vartheta+\rho \bar{V}_{P}}{b}<\frac{F \rho}{b \beta}$ holds if $\vartheta<\frac{F \rho}{\beta}-\rho \bar{V}_{P}$ and there is a non-empty range of parameters for which a BGP exists and for which this condition in addition to the condition $\frac{F \rho}{b \beta}<1$ is satisfied. In Regime $\mathrm{A}, c_{P}$ can be derived from equations 5 and 16 as

$$
c_{P}=\frac{1+F \rho+b \beta-\sqrt{(1+F \rho+b \beta)^{2}-4 b \beta(F \rho+\vartheta-\rho X(1-\beta))}}{2 b \beta}
$$

If $\frac{F \rho}{b \beta}<1, c_{P}<\frac{F \rho}{b \beta}$ requires that $\vartheta<\frac{F \rho}{b \beta}(1+F \gamma)+\rho X(1-\beta)-F \gamma$ holds in addition to the other existence conditions from Proposition 1. (there is a non-empty range of parameters that fulfills all conditions).

b) Assuming that $\frac{F \rho}{b \beta}<1, c_{P}=\frac{F \rho}{b \beta}$ holds if $\gamma=\frac{\frac{F \rho}{b \beta}-\vartheta+\rho X(1-\beta)}{F\left(1-\frac{F \rho}{b \beta}\right)}=\gamma_{P}^{*}$, i.e. if $T_{P}^{*}=$ $\frac{1}{\gamma_{P}^{*}}=\frac{F\left(1-\frac{F \rho}{b \beta}\right)}{\frac{F \rho}{b \beta}-\vartheta+\rho X(1-\beta)}$, so that poor households prefer $T$ to be set equal to this value if it is 
compatible with the existence of a BGP in Regime A. For the welfare analysis it suffices to compare BGP values holding $X$ constant (see Proposition $2 \mathrm{~b}$ with $\gamma=\gamma_{1}=\gamma_{0}$ ).

It is now shown that there are parameter constellations for which $0<\gamma_{P}^{*}<\tilde{\gamma}$, i.e. $\tilde{T}<T_{P}^{*}<\infty$ is an interior solution in Regime A: Suppose that $\gamma_{P}^{*}$ is only slightly positive. Then, Condition D from Proposition 3reduces to $(1-\beta) \rho X-F \rho<\vartheta$ and Condition $\mathrm{E}$ to $\vartheta<(1-\beta) \rho X+\frac{F \rho(1-b)}{b \beta}+\frac{1-b}{\beta}+b$ (the parameter range defined by these two conditions is nonempty under Condition $\mathrm{C}) \cdot \gamma_{P}^{*}$ is positive if $\vartheta<\frac{F \rho}{b \beta}+\rho X(1-\beta)$ and if $\frac{F \rho}{b \beta}<1$ and there is a non-empty range of parameters for which all of the above conditions are satisfied. When $\gamma_{P}^{*}$ is small, $m=\frac{\gamma}{g+\gamma}$ (see equation 8) is close to zero if $g>0$, so that $c_{P}>m$ (Condition A) and therefore $\gamma<\tilde{\gamma}$ (Condition F) holds if $c_{P}>0$. The latter is always satisfied as the term $(F \rho+\vartheta-\rho X(1-\beta))$ that appears under the square root in equation 23 is positive due to Condition E. Therefore, an interior solution exists under these conditions.

Deriving $T_{P}^{*}$ with respect to the different parameters gives $\frac{\partial T_{P}^{*}}{\partial \vartheta}>0, \frac{\partial T_{P}^{*}}{\partial \rho}<0$ and $\frac{\partial T_{P}^{*}}{\partial X}<0$.

$\operatorname{sign}\left(\frac{\partial T_{P}^{*}}{\partial \beta}\right)=\operatorname{sign}\left(F(1-\vartheta)+X\left(b \beta^{2}+F \rho(1-2 \beta)\right)\right)>0$ holds due to the fact that $\frac{F \rho}{b \beta}<1$ and therefore $b \beta>F \rho$ needs to be satisfied in order to obtain an interior solution.

\section{A6: Proof of Proposition 5}

Proof. a): Let us denote the price of an IP protected good that is sold to all except to those households belonging to the poorest $i-1$ income groups by $p_{i}$ and the resulting profits by $\pi_{i}$. Then, $\pi_{i}=\left(p_{i}-b\right)\left(1-\sum_{s=1}^{i-1} \beta_{s}\right)$ if $2 \leq i \leq k$, while profits of a firm selling to all income groups $(i=1)$ are given by $\pi_{1}=p_{1}-b$. As goods are symmetric, profits derived from each non-expired IPR must be the same in equilibrium, i.e., $\pi_{i}=\pi$ must hold. This "equal profit condition" allows the expression of all prices $p_{i}$ as a positive function of $p_{k}$, the price charged by firms that sell exclusively to the richest income group:

$$
p_{i}=b+\frac{\left(p_{k}-b\right)\left(1-\sum_{s=1}^{k-1} \beta_{s}\right)}{1-\sum_{s=1}^{i-1} \beta_{s}}
$$

Prices increase in $i$ and are therefore larger for goods that are sold to fewer income groups. Moreover, all prices depend positively on $p_{k}$ and do not directly depend on income differences between groups, but only on population shares.

Along a BGP, the intertemporal budget constraints of households belonging to income group $i$ are given by:

$$
\begin{aligned}
& \mathrm{BC}(i=1): l_{1}+(r-g) \frac{V(t)}{N(t)}=b m+p_{1}\left(c_{1}-m\right) \\
& \mathrm{BC}(i=2): l_{2}+(r-g) \frac{V(t)}{N(t)}=b m+p_{1}\left(c_{1}-m\right)+p_{2}\left(c_{2}-c_{1}\right) \\
& \mathrm{BC}(\text { general }): l_{i}=l_{i-1}+p_{i}\left(c_{i}-c_{i-1}\right), \text { for } 2 \leq i \leq k
\end{aligned}
$$




$$
\mathrm{BC}(i=k): l_{k}=l_{k-1}+p_{k}\left(1-c_{k-1}\right)
$$

The simplification in the third line is obtained by substituting the budget constraint of group $i-1$ and that in the fourth line by substituting $c_{k}=\frac{C_{k}(t)}{N(t)}=1$. From the fourth line, we obtain $p_{k}=\frac{l_{k}-l_{k-1}}{1-c_{k-1}}$, so that the profits of firms selling exclusively to the richest households are given by $\pi_{k}=\left(\frac{l_{k}-l_{k-1}}{1-c_{k-1}}-b\right)\left(1-\sum_{i=1}^{k-1} \beta_{i}\right)$. Due to free entry, $\frac{\pi}{r+\gamma}=F$ must hold along a BGP, which, together with the familiar Euler equation $r=\rho+g(6)$ gives the free entry condition

$$
\frac{\left(\frac{l_{k}-l_{k-1}}{1-c_{k-1}}-b\right)\left(1-\sum_{i=1}^{k-1} \beta_{i}\right)}{\rho+g+\gamma}=F
$$

This conditions can be plotted as an upward sloping FE curve in $g-c_{k-1}$ - space (with $g$ on the vertical axis) which shifts up if $\gamma$ is decreased (the notation $\gamma=\gamma_{1}$ is used throughout).

Rewriting the intertemporal budget constraint of group $i$ and plugging the equal profit condition (equation 24), with $p_{k}$ replaced by $p_{k}=\frac{l_{k}-l_{k-1}}{1-c_{k-1}}$, gives

$$
c_{i}=c_{i+1}-\frac{l_{i+1}-l_{i}}{p_{i+1}}=c_{i+1}-\frac{l_{i+1}-l_{i}}{b+\left(\frac{l_{k}-l_{k-1}}{1-c_{k-1}}-b\right) \frac{\left(1-\sum_{s=1}^{k-1} \beta_{s}\right)}{\left(1-\sum_{s=1}^{i} \beta_{s}\right)}}
$$

This equation determines the different consumption shares $c_{i}$ (with $1 \leq i \leq k-2$ ) as a function of $c_{k-1}$ and of the labor endowments and population shares. As $p_{i+1}$ depends positively on $c_{k-1}, c_{i+1}-c_{i}=\frac{l_{i+1}-l_{i}}{p_{i+1}}$ decreases if $c_{k-1}$ increases. This implies that, for $l_{i}$ and $\beta_{i}$ given, all consumption shares $c_{i}$ depend positively on $c_{k-1}$ and that a decrease in $c_{k-1}$ leads to an even larger decrease in $c_{i}$ (if $i<k-1$ ). Indeed, the fall in $c_{i}$ caused by the fall in $c_{k-1}$ is the larger in absolute terms the smaller $i$ is, that means the poorer an income group is.

Taking into consideration that the total stock of labor can be either used for R\&D or for the production of final goods, the Resource constraint can be derived as:

$$
g=\frac{1}{F}\left[1-b \sum_{i=1}^{k} c_{i} \beta_{i}\right]
$$

This equation implies a negative relation between $g$ and $c_{k-1}$, as all consumption shares $c_{i}$ (with $1 \leq i \leq k-2$ ) depend positively on $c_{k-1}$ (due to equation 26). The resource constraint therefore defines a downward-sloping RC curve in $g-c_{k-1}$ - space which is independent of $\gamma$.

The BGP values of $g$ and $c_{k-1}$ are determined by equations 25 and 27 ), i.e. by the intersection of the $\mathrm{FE}$ and the $\mathrm{RC}$ curve. As the FE curve shifts up when $\gamma$ declines and as the $\mathrm{RC}$ curve is independent of $\gamma$, a decrease in $\gamma$ therefore increases $g$ and reduces 
$c_{k-1}$. Due to equation 26, this decrease in $c_{k-1}$ implies a decrease in all other consumption shares $c_{i}$ that is greater in absolute value the poorer a group is (the lower $i$ is). A BGP along which $m<c_{1}$ holds exists if $\gamma$ is sufficiently small (remember that $m=\frac{\gamma}{g+\gamma}$ as in equation 8), i.e. if $T$ is sufficiently large.

b) As the state variables $m$ and $V$ do not enter into equations 25, 26 and 27, there are no transitional dynamics and $p_{i}, c_{i}$, and $g$ immediately jump to their new BGP values ${ }^{59}$. Given that $V_{i}=V$, an unexpected change in the length of IP protection on previously issued IPRs (i.e. a change in $\gamma_{0}$ ) which affects the value of initial wealth $V_{i}=V$ affects all households symmetrically. As such a change does not affect the absolute expenditure differences between income groups (which are given by $l_{i}-l_{i-1}$, as can be inferred form the intertemporal budget constraints) and therefore does not affect prices $p_{i}$ and profits, it has no impact on the free entry condition (equation 25). As the resource constraint (equation 27) does not depend on $\gamma_{0}$ either, $g$ and $c_{i}$ therefore only depend on the hazard rate $\gamma_{1}=\gamma$ with which newly issued IPRs expire and not on $\gamma_{0}$. This is a generalization of the result that was formally derived in the case of two income groups where $V_{i}=V$ holds if $X=0$ (see Proposition 2).

c) Intertemporal utilities along the BGP are given by

$$
U_{i}(\tau)=\int_{t=\tau}^{\infty} \ln \left(c_{i} N(t)\right) e^{-\rho(t-\tau)} d t=\frac{\ln (N(\tau))}{\rho}+\frac{\ln \left(c_{i}\right)}{\rho}+\frac{g}{\rho^{2}}
$$

As there are no transitional dynamics for the variables $c_{i}$ and $g$, a household in income group $i$ therefore benefits from an (unexpected) reduction in the length of IP protection (i.e. an increase $\gamma$ ) if $\frac{\partial U_{i}}{\partial \gamma}=\frac{1}{\rho}\left[\frac{1}{c_{i}} \frac{\partial c_{i}}{\partial \gamma}+\frac{1}{\rho} \frac{\partial g}{\partial \gamma}\right]>0$ and benefits from an increase in IP protection if $\frac{\partial U_{i}}{\partial \gamma}<0$. $\frac{\partial U_{i}}{\partial \gamma}>0$ holds if $\frac{1}{c_{i}} \frac{\partial c_{i}}{\partial \gamma}>-\frac{1}{\rho} \frac{\partial g}{\partial \gamma}>0$, which is more likely satisfied the smaller $i$ and the poorer an income group is, as $c_{i}$ increases in $i$ and as $\frac{\partial c_{i}}{\partial \gamma}(>0)$ decreases in $i$ (see above) ${ }^{60}$. As $c_{k}=1$ and $\frac{\partial c_{k}}{\partial \gamma}=0, \frac{\partial U_{k}}{\partial \gamma}<0$ always holds, so that households in the richest income group always prefer infinite IP protection, i.e. $\gamma_{k}^{*}=0$.

Suppose that there is a value $\gamma_{n}^{*}>0\left(\right.$ or $\left.T_{n}^{*}<\infty\right)$ for which the utility of an income group $n$ (with $1<n<k$ ) is maximal and for which a BGP with $c_{1}>m$ exists. Then, $\frac{\partial U_{n}}{\partial \gamma}\left(\gamma=\gamma_{n}^{*}\right)=0$ must hold as $U_{i}$ is continuous and differentiable in $\gamma$ along such a BGP. As $\frac{\partial U_{i}}{\partial \gamma}$ decreases in $i$, poorer households $(i<n)$ then benefit from a marginal increase

\footnotetext{
${ }^{59}$ For such an instantaneous transition to be feasible, the budget constraint of the poorest income group (BC $(i=1))$ which contains the state variables $V$ and $m$ must be satisfied during the whole transition phase. This is not verified here and would require an analysis similar to the one undertaken in the case of two income groups (see the proof of Proposition 2).

${ }^{60}$ Even if the consumption shares $c_{i}$ of all income groups were reduced by the same absolute amount if $\gamma$ increased (i.e. if $\frac{\partial c_{i}}{\partial \gamma}$ was independent of $i$ ), poorer households would still be more likely to suffer from such a policy change. The reason for this is that intertemporal utility is concave in current consumption so that richer households are willing to reduce their consumption by a larger absolute amount than poorer households in order to obtain a certain increase in the rate of growth.
} 
in $\gamma$ above the level $\gamma_{n}^{*}$ (as $\frac{\partial U_{i}}{\partial \gamma}\left(\gamma=\gamma_{n}^{*}\right)>0$ for $\left.i<n\right)$, while richer households $(i>n)$ benefit from a marginal reduction in $\gamma$ below the level $\gamma_{n}^{*}\left(\right.$ as $\frac{\partial U_{i}}{\partial \gamma}\left(\gamma=\gamma_{n}^{*}\right)<0$ for $\left.i>n\right)$.

In order to avoid messy calculations, it is not shown here that $\frac{\partial^{2} U_{i}(\gamma)}{\partial \gamma^{2}}<0$ globally holds. Therefore, it is unclear whether there might in fact be several local maxima for which $\frac{\partial U_{i}}{\partial \gamma}=0$ holds. Given that $\gamma_{n}^{*}$ is the global maximum for group $n$ (out of several local maxima) this, however, does not imply that households with $i<n$ might actually benefit from a non-marginal reduction in $\gamma$ below the level $\gamma_{n}^{*}$. The reason for this is the following: as $\frac{\partial U_{i}}{\partial \gamma}$ decreases in $i$, we can write $U_{n}=U_{i}+f_{i n}(\gamma)$ with $f_{i n}(\gamma)>0$ if $i<n$ and $\frac{\partial f_{i n}(\gamma)}{\partial \gamma}<0$. Given that $U_{n}$ is maximal for the value $\gamma_{n}^{*}, U_{i}=U_{n}-f_{i n}(\gamma)$ can therefore not be maximal for a value $\tilde{\gamma}<\gamma_{n}^{*}$ (which might be a local maximum) as $f_{i n}(\tilde{\gamma})>f_{i n}\left(\gamma_{n}^{*}\right)$. A symmetric argument can be used to show that it is also not possible for households with $i>n$ to benefit from a non-marginal increase in $\gamma$ above the level $\gamma_{n}^{*}$. Therefore, an income group $i<n$ prefers a shorter duration of IP protection than group $j$ (i.e. $T_{i}^{*}<T_{n}^{*}$ and $\gamma_{i}^{*}>\gamma_{n}^{*}$ ), and an income group $i>n$ prefers a longer duration of IP protection than group $n$ (i.e. $T_{i}^{*}>T_{n}^{*}$ and $\gamma_{i}^{*}<\gamma_{n}^{*}$ ).

The analysis conducted so far was based on the assumption that an interior solution $\gamma_{n}^{*}>0$ (i.e. $T_{n}^{*}<\infty$ ) which is compatible with the existence of an equilibrium exists. There is indeed a non-empty range of parameters for which such a solution exists: As has been shown in Proposition 4b, there are conditions under which an interior solution exists in the case of two income groups, $R$ and $P$. Suppose now that more income groups $i$ are added and that the per capita incomes of these groups lie below that of group $R$ and that their population shares $\beta_{i}$ are very small. This does not affect equations 27 and 25 (the latter defining $g$ as a function of $c_{P}$ ) in a discontinuous way, so that $c_{P}$ and $g$ do not change discontinuously. Therefore, an interior solution $\gamma_{P}^{*}>0$ (i.e. $T_{n}^{*}<\infty$ ) exists if parameters lie in the range derived in the proof of Proposition $4 \mathrm{~b}$ and if the additional groups $i$ are only endowed with a small fraction of the total labor endowment. Then, $\gamma_{i}^{*}>\gamma_{P}^{*}\left(T_{i}^{*}<T_{P}^{*}\right)$ holds if group $i$ is poorer than group $P$ and $\gamma_{i}^{*}<\gamma_{P}^{*}\left(T_{i}^{*}>T_{P}^{*}\right)$ if group $i$ is richer than group $P$. 


\section{For online publication: Appendix B}

\section{B1: Selected "innovative" goods}

In the following, the list of goods from the CEX interview survey (INTR) which are classified as "innovative" is provided. The universal classification codes (UCC) are added in brackets. In cases where two or more UCCs are grouped together ${ }^{61}$, the group is counted as one innovative good which is assumed to be consumed by each household that consumes at least one of the UCC items within the group:

Computers, computer systems, and related hardware for non-business use (690111); computer information services (690114); internet services away from home (690116); portable memory (690117); digital book readers (690118); computer software (690119); computer accessories (690120); applications, games, and ringtones for devices (310400); calculators (690220); watches (UCC: 430110); electric personal care appliances (640420); photographic equipment (610230);

Telephones and accessories (320232); telephone answering devices (690210); voice over IP service (270105); televisions (310130; 310140); VCR, video disc player, video camera, and camcorder (310210); Video cassettes, tapes, and discs (310220); rental of video cassettes, tapes, and discs (620912); video game software (310231); rental of video software (620918); video game hardware/ accessories (310232); rental of video hardware/ accessories (620917); online entertainment and games (620930); streaming or downloaded video files (310240);

Radios (310311); digital audio players (310314); sound components, component systems, and compact disc sound systems (310320); accessories and other sound equipment including phonographs (310333); records, CDs, audio tapes (310340); streaming or downloaded audio files (310350); cable, satellite, or community antenna service (270310); satellite radio service (270311); satellite dishes (310334);

New cars (450110); new trucks (450210); new motorcycles (450220); purchase of motor home (600141); purchase of other vehicle (600142); aircraft (450900); vehicle products and services (480212); vehicle parts, equipment, and accessories (480213); vehicle audio equipment (480214); vehicle video equipment (480215); global positioning services (520560); airline fares (530110).

Built-in dishwasher, garbage disposal, or range hood (220612); supplies for electrical work, heating or air conditioning (240321; 240322; 240323); refrigerator or home freezer (300111; 300112); clothes washer (300211; 300212); clothes dryer (300221; 300222); stoves, ovens (300311; 300312); microwave (300321; 300322); dishwasher (300331; 300332); window air conditioner $(300411 ; 300412)$; power tools $(320420)$; electric floor clean-

\footnotetext{
${ }^{61}$ This is mainly done when there are different UCCs depending on whether a good (e.g. a fridge) is purchased by a homeowner or a renter.
} 
ing equipment (320511); sewing machines (320512); small electric kitchen appliances (320521); portable heating and cooling equipment (320522); other household appliances (690244; 690245).

\section{B2: First best}

The objective function of a social planner who maximizes the weighted sum of intertemporal utilities $\left(U_{i}(\tau)=\int_{t=\tau}^{\infty} \ln \left(C_{i}(t)\right) e^{-\rho(t-\tau)} d t\right)$ and discriminates between rich and poor households is given by

$$
W(\tau)=\alpha(1-\beta) U_{R}(\tau)+(1-\alpha) \beta U_{P}(\tau)
$$

with $\alpha$ and $1-\alpha$ denoting the welfare weights for a rich and a poor household $\left(\alpha>\frac{1}{2}\right)$. The planner has to take the following resource constraint into account:

$$
\dot{N(t)}=\frac{N(t)}{F}\left[1-b \beta \frac{C_{P}(t)}{N(t)}-b(1-\beta) \frac{C_{R}(t)}{N(t)}\right]
$$

To solve the problem, we can write the Hamiltonian:

$$
H(t)=e^{-\rho(t-\tau)}\left[\alpha(1-\beta) \ln \left(C_{R}(t)\right)+(1-\alpha) \beta \ln \left(C_{P}(t)\right)\right]+\mu(t)[\dot{N}(t)]
$$

Suppose first that $C_{i}(t)<N(t)$ holds (interior solutions). Then, the first order conditions $\frac{\partial H(t)}{\partial C_{i}(t)}=0$ give: $C_{R}(t)=\frac{\alpha F e^{-\rho(t-\tau)}}{b \mu(t)}$ and $C_{P}(t)=\frac{(1-\alpha) F e^{-\rho(t-\tau)}}{b \mu(t)}$ (the second order conditions are satisfied in all cases). Plugging these expressions into the resource constraint gives $\dot{N}(t)=\frac{N(t)}{F}-e^{-\rho(t-\tau) \frac{(\alpha+\beta-2 \alpha \beta)}{\mu(t)}}$ and plugging them into the Hamiltonian, deriving with respect to $N(t)$ and setting the derivative equal to $-\dot{\mu}$ gives the second differential equation $\dot{\mu}=-\frac{\mu}{F}$. The two differential equations are solved by the functions: $\mu(t)=\frac{\alpha+\beta-2 \alpha \beta}{\rho N(\tau)} e^{-\frac{1}{F}(t-\tau)}$ and $N(t)=N(\tau) e^{\left(\frac{1}{F}-\rho\right)(t-\tau)}$. These functions are the optimal trajectories as the maximized Hamiltonian with $\mu(t)=\frac{\alpha+\beta-2 \alpha \beta}{\rho N(\tau)} e^{-\frac{1}{F}(t-\tau)}=\frac{\alpha+\beta-2 \alpha \beta}{\rho N(t)} e^{-\rho(t-\tau)}$ plugged in is concave in $N(t)$ and as $\lim _{t \rightarrow \infty} N(t) \mu(t)=0$, so that the transversality condition is satisfied. We can therefore derive the optimal consumption paths as: $C_{R}^{*}(t)=$ $\frac{\alpha F \rho N(\tau) e^{\left(\frac{1}{F}-\rho\right)(t-\tau)}}{b(\alpha+\beta-2 \alpha \beta)}$ and $C_{P}^{*}(t)=\frac{(1-\alpha) F \rho N(\tau) e^{\left(\frac{1}{F}-\rho\right)(t-\tau)}}{b(\alpha+\beta-2 \alpha \beta)}$. These interior solutions result if $\frac{C_{R}^{*}(t)}{N(t)}=\frac{\alpha F \rho}{b(\alpha+\beta-2 \alpha \beta)}<1$ and if $g^{*}=\frac{1}{F}-\rho>0$ (note that $g^{*}$ is independent of $\alpha$ in this case).

The more interesting and relevant ${ }^{62}$ case of a corner solution $C_{R}^{*}(t)=N(t)>N_{P}^{*}(t)$

\footnotetext{
${ }^{62}$ The result that an interior solution $C_{P}(t)<C_{R}(t)<N(t)$ where not all invented goods are immediately produced can be optimal is driven by the technical assumption that an increase in $N(t)$ increases labor productivity in all sectors. Such a situation can, however, not occur in a market equilibrium with
} 
results if $\frac{\alpha F \rho}{b(\alpha+\beta-2 \alpha \beta)} \geq 1>\frac{(1-\alpha) F \rho}{b(\alpha+\beta-2 \alpha \beta)}$. Then, the optimal consumption paths are

$$
N_{R}^{*}(t)=N(t)=N(\tau) e^{\left(\frac{1-(1-\beta) b}{F}-\frac{\beta(1-\alpha) \rho}{\alpha+\beta-2 \alpha \beta}\right)(t-\tau)}
$$

and

$$
N_{P}^{*}(t)=\frac{(1-\alpha) F \rho}{b(\alpha+\beta-2 \alpha \beta)} N(\tau) e^{\left(\frac{1-(1-\beta) b}{F}-\frac{\beta(1-\alpha) \rho}{\alpha+\beta-2 \alpha \beta}\right)(t-\tau)}
$$

and the optimal rate of growth $g^{*}=\frac{1-(1-\beta) b}{F}-\frac{\beta(1-\alpha) \rho}{\alpha+\beta-2 \alpha \beta}$ increases in $\alpha$.

\section{B3: Appropriating R\&D spillovers through extended IP protection}

It is now assumed that IPRs grant forward protection, allowing previous innovators to charge licensing fees from future innovators who build on the knowledge they created. For simplicity, the case of infinite IP protection against imitation $(\gamma=0)$ is considered. As all previously invented goods are symmetric, each holder of an IPR that was granted in the past obtains the same licensing payment from an innovator who enters the market.

Let us assume that an innovator is required to pay total licensing fees equal to $Q$ upon entry. As the mass of innovators in period $t$ is given by $N(t) g$, each of the $N(t)$ holders of previously granted IPRs receives licensing income equal to $g Q$ in any period $t$. As per period profits $\pi$ from the sale of a good are constant along a BGP, the value of an innovation is given by $V=\frac{\pi}{r}+\frac{g Q}{r}-Q$. Plugging in $r=\rho+g>g$ (equation 6), one clearly sees that $V$ decreases in $Q$. Given that $Q<\frac{\pi}{\rho}$, the free-entry curve (that is defined by the equation $V=\frac{\pi}{r}+\frac{g Q}{r}-Q=F$ ) is upward-sloping in $g$ - $n$ - space (note that $\pi$ depends positively on $n$ ) and moves to the right if $Q$ increases, so that $g$ declines in the size of the licensing fee $Q$.

The reason why an increase in $Q$ decreases the rate of growth is that it makes profits for innovators more backloaded ${ }^{63}$. Therefore, $Q$ would need to rise faster than the rate of interest in order to increase innovation incentives. But this is not possible in the long run as licensing payments have to be paid out of total profits which grow at a rate that is lower than the rate of interest.

positive growth.

${ }^{63}$ This analysis is related to O' Donoghue and Zweimüller (2004) who identify a similar backloading effect of forward patent protection in a quality-ladder growth model. In a setup similar to a product variety model, Llanes and Trento (2012) find that it would indeed be optimal to pay innovators a subsidy when they innovate, and to tax them when subsequent innovations are introduced. 


\section{B4: Finite length of IP protection and varying enforce- ment probability}

So far, it was assumed that IP protection expires at a constant stochastic rate. Suppose now that IPRs instead expire after $\hat{T}$ periods (finite patent length). Then, the value of an innovation along a BGP is given by $Z(t)=\int_{s=t}^{t+\hat{T}} \pi e^{-r(s-t)} d s=\frac{\pi\left(1-e^{-r \hat{T}}\right)}{r}$.

Considering Regime A in which $\pi=(1-\beta)\left(p_{R}-b\right)$, plugging in equations 15 and 6 (which remain unchanged) and setting $Z(t)=F$ gives the new free entry condition

$$
\frac{(1-\beta)\left(\frac{\frac{1-\vartheta}{1-\beta}+\rho X}{1-c_{P}}-b\right)\left(1-e^{-(\rho+g) \hat{T}}\right)}{\rho+g}=F
$$

This condition again gives a positive relation between $g$ and $c_{P}$ and between $g$ and $\hat{T}^{64}$. As the resource constraint is unchanged, the qualitative effects of increasing $\hat{T}$ are therefore the same as those of increasing $T$ (i.e. of reducing $\gamma$ ) when BGPs are compared. Moreover, the effects of inequality on growth and the welfare effects remain unchanged. As the analysis of transitional dynamics is more involved if IPRs are of finite duration than in the case of stochastic IP expiration (see footnote 9 for references) is not carried out here.

If IPRs are infinitely lived, but only enforced with probability $\Delta$ at the time of invention (this is how Bernal Uribe (2012) parametrizes the strength of IP protection), the free entry condition can be derived as

$$
\frac{(1-\beta)\left(\frac{\frac{1-\vartheta}{1-\beta}+\rho X}{1-c_{P}}-b\right) \Delta}{\rho+g}=F
$$

This again gives a positive relation between the strength of IP protection, $\Delta$, and $g$. Also with this parametrization of the strength of IP protection, the qualitative results stay the same when BGPs are compared.

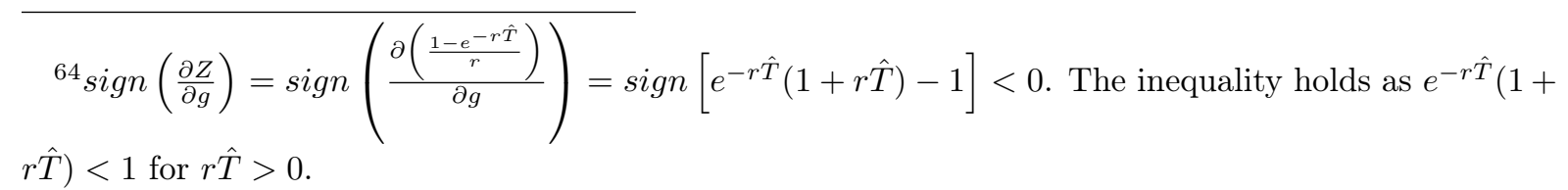




\section{B5: Hierarchical preferences}

In Föllmi and Zweimüller (2006), instantaneous utility is given by $u(t)=\int_{j=0}^{\infty} j^{-\theta} c(j, t) d j$ with $\theta \in[0,1)$. When $\theta>0$, preferences are hierarchical and households attach a lower weight $j^{-\theta}$ (i.e. a lower consumption priority) to goods with a higher index $j$. In a previous version of this paper ${ }^{65}$, I analyzed the effects of IP protection of finite duration $\hat{T}$ within this setup, simply comparing BGPs and assuming that $V_{R}=V_{P}$ (i.e. $X=0$ ) holds:

Given that $\theta$ is sufficiently small ("flat" hierarchy) there is again a regime in which $M<C_{P}<C_{R}=N$ holds and in which the qualitative effects that changes in $\hat{T}$ (and in inequality) have on growth and welfare are the same as those that they have in Regime A when goods are symmetric $(\theta=0)$. When $\theta$ is sufficiently large ("steep hierarchy") an additional regime can arise in which $M<C_{P}<C_{R}<N$ holds and in which there might be multiple equilibria (like in Föllmi and Zweimüller, 2006). Given that the economy is in the stable high-growth equilibrium, an increase in $\hat{T}$ is associated with an increase in $g$ and with a reduction in $C_{P}$ and $C_{R}$, with the former reduction being larger (in absolute terms) than the latter. Consequently, rich households still prefer a larger value of $\hat{T}$ (when BGPs are compared) ${ }^{66}$.

\section{B6: The effect of inequality on growth}

Suppose that labor endowments (or wage incomes, that are equal to $l_{i} N(t)$ ) are redistributed from poor to rich households in such a way that the difference $l_{n+1}-l_{n}$ increases for income group $n<k-1$ and does not change for any other group $i \neq n^{67}$. This does not affect the free entry condition 25 as $l_{k}-l_{k-1}$ is constant, but it shifts the resource constraint curve (equation 27) outward. The latter can be seen from equation 26 : $c_{i}=c_{i+1}-\frac{l_{i+1}-l_{i}}{p_{i+1}}$ which indicates that - for $c_{k-1}$ given - resources are freed for R\&D and growth as the consumption shares $c_{i}$ of groups with index $i \leq n$ are reduced while those

\footnotetext{
${ }^{65}$ The title of this version was "Do poorer people like shorter patents?", see https://gremaq.univtlse1.fr/seminaires/kiedaisch.pdf

${ }^{66}$ With hierarchical preferences, a household's willingness to pay $z_{i}$ falls in the index $j$, implying that also per period profits are lower for IP protected goods with a higher index $j$ (i.e. for more luxurious goods). Consequently, innovators always invent the goods with the lowest index $j$ among the not yet invented goods. When IPRs expire after $\hat{T}$ periods, the goods on which IP protection has expired are consequently the most valuable ones (i.e. basic need goods with a low value of $j$ ) and both rich and poor households prefer to consume these goods first. When IPRs instead expire stochastically at rate $\gamma$, there can be luxurious goods on which IPRs have expired and poor households might prefer to purchase IP protected basic need goods instead of these goods. Consequently, rich and poor households might not purchase the same variety of non-IP protected goods and the effects of reducing $\gamma$ (i.e. of increasing $T$ ) might not coincide with those of increasing $\hat{T}$.

${ }^{67}$ This implies that the incomes of groups with index $i \leq n(>n)$ all decrease (increase) by the same absolute amount.
} 
of groups with index $i>n$ do not change. Therefore, such a redistribution from poor to rich households increases the rate of growth $g$ and increases $c_{k-1}$. If $l_{k}-l_{k-1}$ increases but all other $l_{i+1}-l_{i}$ are unchanged, the FE curve shifts up in $g-c_{k-1}$ - space while the RC curve is unchanged so that $g$ increases and the consumption shares of all but the richest group $k$ decrease. Generalizing the argument, we obtain:

Proposition 6. Suppose that $V_{i}(\tau)=V(\tau)$ and that there is a BGP in which $c_{1}>m$ holds. If income is redistributed in such a way that $l_{n+1}-l_{n}$ increases for some $i=$ $n$ and does not decrease for any other $i \neq n$, the BGP growth rate $g$ increases. If $l_{i+1}-l_{i}$ increases for $i=n$ but does not change for any other $i \neq n, g$ increases and the consumption shares $c_{i}$ of all groups $i \leq n$ decrease while the consumption shares of the richer groups $n<i<k$ increase ( $c_{k}=1$ always holds).

The intuition for this result is the following: if, for given prices, income is redistributed from a poor to a rich household, the consumption share $c_{i}$ of the poor household decreases more than that of the rich increases, as the rich household needs to pay higher prices for additional goods than the poor one does. Therefore, profits and the incentives to innovate increase (or, put differently: less labor in the production sector is needed which can now be used in the $R \& D$ sector).

Given that the consumption share of a poor household has been reduced due to such an increase in inequality, this household now prefers a weaker level of IP protection than before, as increasing $g$ by increasing IP protection becomes more costly in terms of current consumption if $c_{i}$ is lower (because intertemporal utility is linear in $g$ but concave in $c_{i}$; see the proof of Proposition 5c). If the median voter decides about the strength of IP protection (and no transfer payments are used), an increase in inequality that reduces the consumption share $c_{m}$ of the median voter will therefore be accompanied by a reduction in the strength of IP protection.

\section{B7: Restricted monopoly power}

In all regimes, the parametrization $V_{R}(t)=V_{P}(t)+X N(t)$ and the notation $c_{R}=\frac{C_{R}}{N}$ is used.

\section{Regime C1}

In Regime $\mathrm{C} 1$ ), rich households consume some traditionally produced goods at price $\Omega$, so that their willingness to pay is given by $z_{R}(t)=\Omega$. As $C_{P}(t)<N(t)$ and as poor households prefer to consume the cheaper goods on which IP protection has expired, they do not consume the whole variety of IP protected goods in this regime. The IP protected goods that are exclusively sold to rich households are sold at the price $p_{R}(t)=z_{R}(t)=\Omega$. 
In the following, only the case is considered in which poor households consume some but not all of the IP protected goods (meaning that $M(t)<C_{P}(t)<N(t)$ ). Symmetry in profits then requires that IP holding firms that sell to both income groups charge the price

$$
p_{P}(t)=z_{p}(t)=\beta b+(1-\beta) \Omega
$$

Proposition 7. a) Along the BGP, $c_{P}=\frac{1}{b \beta+\Omega(1-\beta)}[\vartheta+F(\rho+\gamma)-(1-\beta) X \rho]$, $g=\frac{(1-\beta)(\Omega-b)}{F}-\rho-\gamma$ and $c_{R}=\frac{1}{b \beta+\Omega(1-\beta)}[\vartheta+F(\rho+\gamma)-(1-\beta) X \rho]+\frac{1-\vartheta}{(1-\beta) \Omega}+\frac{X \rho}{\Omega}$. The $B G P$ exists if parameters are such that $g>0, m=\frac{\gamma}{g+\gamma}<c_{P}<1, c_{R}>1$ and $X<\tilde{X}=\frac{(1-m) F}{1-\beta}$ hold.

b) $g, c_{P}$ and $c_{R}$ immediately jump to their new BGP values when there is a change in $\gamma=\gamma_{1}=\gamma_{0}$ or in another exogenous parameter (including $X$ ).

c) While rich households always prefer infinite IP protection $\left(\gamma_{R}^{*}=0\right)$, poor households prefer finite IP protection if $(1-\beta) X \rho-\vartheta>0$ (i.e. if inequality is sufficiently large) and infinite protection if $(1-\beta) X \rho-\vartheta<0$. Given that the value of $T \equiv \frac{1}{\gamma}$ poor households prefer is finite and compatible with the existence of a BGP in Regime $C 1$, it is given by $T_{P}^{*}=\frac{F}{(1-\beta) X \rho-\vartheta}$.

Proof. a) The demand for production labor is given by

$L_{D}(t)=\int_{j=0}^{C_{P}(t)}\left(\frac{b}{N(t)}\right) 1 d j+\int_{j=C_{P}(t)}^{N(t)}\left(\frac{b}{N(t)}\right)(1-\beta) d j+\int_{j=N(t)}^{C_{R}(t)}\left(\frac{\Omega}{N(t)}\right)(1-\beta) d j=b \beta c_{P}+$ $b(1-\beta)+\Omega\left(c_{R}-1\right)(1-\beta)$

The resource constraint can therefore be derived as

$$
g(t)=\frac{1}{F}\left[1-b \beta c_{P}-b(1-\beta)-\Omega(1-\beta)\left(c_{R}-1\right)\right]
$$

Along a BGP, the intertemporal budget constraint of a poor household is given by

$$
\frac{N(\tau) \vartheta}{r-g}+V_{P}(\tau)=\frac{N(\tau)}{r-g}\left[m b+[b \beta+\Omega(1-\beta)]\left(c_{P}-m\right)\right]
$$

and that of a rich household by

$$
\begin{aligned}
& \frac{N(\tau) \frac{1-\beta \vartheta}{1-\beta}}{r-g}+V_{P}(\tau)+X N(\tau)= \\
& \frac{N(\tau)}{r-g}\left[m b+[b \beta+\Omega(1-\beta)]\left(c_{P}-m\right)+\Omega\left(c_{R}-c_{P}\right)\right]
\end{aligned}
$$

Along a BGP, the free entry condition is given by $Z=\frac{(1-\beta)(\Omega-b)}{r+\gamma}=F$. Plugging in equation $6(r=\rho+g)$, this condition pins down the BGP growth rate as

$$
g=\frac{(1-\beta)(\Omega-b)}{F}-\rho-\gamma
$$


Combining equations 32, 29, 30 and 31 allows the derivation of the BGP values of $c_{P}$ and $c_{R} . X<\tilde{X} \equiv \frac{(1-m) F}{1-\beta}>0$ again needs to hold in order to ensure that $V_{P}(t)>0$.

b) In order to show that $g, c_{P}$, and $c_{R}$ immediately jump to their new BGP values it needs to be checked that along the transition path (where $V$ and $M$ adjust sluggishly) the intertemporal budget constraint of a poor household is satisfied if $g, c_{P}$ and $c_{R}$ adjust immediately and if $X$ is constant. The budget constraint is given by

$$
\begin{aligned}
& \frac{N(\tau) \vartheta}{r-g}+V_{P}(\tau)=\frac{N(\tau) c_{P}(b \beta+\Omega(1-\beta))}{r-g}+ \\
& \qquad \int_{t=\tau}^{\infty}[M(t)(b-b \beta-\Omega(1-\beta))] e^{-r(t-\tau)} d t
\end{aligned}
$$

Plugging in equations 6 and 32, and equation 20 and $V_{P}(\tau)=N(\tau)\left[\left(1-m_{o}\right) F-(1-\beta) X\right]$ from the proof of Proposition 2, it can be shown that this equation is indeed satisfied. The intertemporal budget constraint of a rich household automatically holds as $c_{R}$ and $c_{P}$ are constant during the transition.

c) Intertemporal utility of a household in group $i$ is given by $U_{i}(\tau)=\frac{\ln N(\tau)}{\rho}+\frac{\ln c_{i}}{\rho}+\frac{g}{\rho^{2}}$. Plugging in the BGP values for $c_{P}, c_{R}$ and $g$, it can be shown that $U_{P}$ and $U_{R}$ are concave in $\left.\gamma \cdot \frac{\partial U_{P}(\tau)}{\partial \gamma}\right|_{\gamma=0}>0$ holds if $(1-\beta) X \rho-\vartheta>0$ and $U_{P}(\tau)$ is maximal if $\gamma=\max \left\{\frac{(1-\beta) X \rho-\vartheta}{F} ; 0\right\}$. Given that $\frac{(1-\beta) X \rho-\vartheta}{F}$ is positive and that this value of $\gamma$ is compatible with a BGP in Regime $\mathrm{C} 1, T_{P}^{*}=\frac{1}{\gamma_{P}^{*}}=\frac{F}{(1-\beta) X \rho-\vartheta}$ is therefore the length of IP protection preferred by poor households.

As $\left.\operatorname{sign} \frac{\partial U_{R}(\tau)}{\partial \gamma}\right|_{\gamma=0}=\operatorname{sign}[-[1-\vartheta+(1-\beta) X \rho] b \beta-(1-\beta) \Omega]<0$, rich households prefer infinite IP protection.

In this regime, an increase in inequality resulting from an increase in $\beta$ reduces the rate of growth (by reducing the number of rich households that buy IP protected goods at price $\Omega$ and by reducing $\left.p_{P}(t)=z_{p}(t)=\beta b+(1-\beta) \Omega\right)$, while, unlike in Regimes A and $\mathrm{B}, g$ does not depend on $\vartheta$ and $X$ as these parameters only affect consumption shares $c_{i}$ but not prices.

\section{Length vs breadth of IP protection}

Proposition 8. Rich households prefer long (high $T$ and low $\gamma$ ) and narrow (low $\bar{p}$ ) IPRs while poor households prefer short (low T and high $\gamma$ ) and broad (high $\bar{p}$ ) IPRs in order to provide innovation incentives that lead to a given rate of growth. When IP policy is symmetrically changed for new and previously issued IPRs $\left(\gamma=\gamma_{1}=\gamma_{0}\right.$ and $\left.\bar{p}=\bar{p}_{1}=\bar{p}_{o}\right)$, $c_{P}$ and $c_{R}$ immediately jump to their new BGP values, while $X$ stays constant.

Proof. Replacing $\Omega$ by $\bar{c}$ in the intertemporal budget constraint of poor households allows to derive their consumption share as 
$c_{P}=\frac{1}{b \beta+\bar{p}(1-\beta)}[\vartheta+F(\rho+\gamma)-(1-\beta) X \rho]$. Replacing $\Omega$ by $\bar{c}$ in the free entry condition gives the following positive relation between $\gamma$ and $\bar{p}$ when the rate of growth is fixed at the level $g=\bar{g}: \gamma=\frac{(1-\beta)(\bar{p}-b)}{F}-\rho-\bar{g}$. Plugging this into the expression for $c_{P}$ and deriving with respect to $\bar{p}$, we obtain

$$
\left.\operatorname{sign} \frac{\partial c_{P}}{\partial \bar{p}}\right|_{g=\bar{g}}=\operatorname{sign}\{b-\vartheta+(1-\beta) X \rho+F \bar{g}\}>0
$$

The inequality has to hold for $c_{P}<1$. Consequently, poor households prefer obtaining a given rate of growth through broad (high $\bar{p}$ ) and short (high $\gamma$ and low $T$ ) IPRs, as they lead to a larger consumption share $c_{P}$. For rich households, the opposite holds as, due to the resource constraint, $c_{R}$ has to fall when $c_{P}$ rises and when $g=\bar{g}$ is held constant. When $\gamma$ and $\bar{p}$ are adjusted at point in time $t=\tau$ (for new and previously issued IPRs) and constant thereafter, there are again no transition dynamics for $c_{P}$ and $c_{R}$, as the value of initial wealth stays unchanged in $t=\tau$ and as the intertemporal budget constraints are satisfied during the transition period as shown in the proof of Proposition 7 (with $\Omega$ replaced by $\bar{p}$ at the appropriate places).

The same reasoning can be applied to a static model of monopolistic competition in which the variety of goods is exogenously given. Keeping total monopoly profits and their distribution constant, poor households then prefer an antitrust policy that is very strict in some sectors (i.e. marginal cost pricing) but lax in others (unconstrained monopoly power) relative to one that increases competition (and reduces prices) a little bit in many sectors and that rich households prefer.

In a previous version of the paper (Kiedaisch, 2009) in which instantaneous utility is given by

$$
u_{i}\left(x_{i}(t), c_{i}(j, t)\right)=x_{i}(t)^{\nu} \int_{j=0}^{\infty} c_{i}(j, t) d j
$$

with $\nu>0$ and $x_{i}(t)$ denoting the consumption of non-innovative goods, it is shown (assuming that $X=0$ ) that long and narrow IPRs are efficient in the regime where $M(t)<C_{P}(t)<C_{R}(t)=N(t)$. This means that for a given rate of growth, both groups can benefit if $\gamma$ is reduced to zero (infinite IP protection) and if at the same time $\bar{p}$ is reduced and if rich households compensate poor households with transfer payments. The reason for this is that long and narrow IPRs reduce markups and the consumption of the non-innovative goods which is inefficiently high when IP protected goods are sold at a markup. This argument is similar to that of Gilbert and Shapiro (1990) who show (in a partial equilibrium model) that it is optimal to have an infinite patent life but a narrow patent breadth (scope) to minimize the deadweight losses associated with a given strength of R\&D incentives. 


\section{Regime C2}

In Regime $\mathrm{C} 2$, poor households consume all goods that are produced with innovative technologies $\left(C_{P}(t)=N(t)\right.$, i.e. $\left.c_{P}=1\right)$ and the prices of these goods are endogenous and given by $p_{L}(t)=z_{L}(t)<\Omega$. Rich households in addition purchase some traditionally produced goods at price $\Omega$, so that $z_{R}(t)=\Omega$.

Proposition 9. a) $A B G P$ in Regime C2 exists if $\vartheta-b-\rho(1-\beta) X>0, F(\rho+\gamma)+$ $\vartheta-\rho(1-\beta) X<\Omega<\frac{F(\rho+\gamma)+\vartheta-b \beta}{(1-\beta)}-\rho X$ and if $X<\tilde{X}>0$. Along a BGP, $g=$ $\frac{1}{F}(\vartheta-b-\rho(1-\beta) X)$ and $c_{R}=1+\frac{1-\vartheta+\rho(1-\beta) X}{(1-\beta) \Omega}$.

b) Suppose that the economy is on a BGP and that at date $t=t_{0}$ there is an unexpected change in the strength of IP protection as described in Proposition 2. Then, $g$ and $c_{R}$ immediately jump to their new BGP values and $X$ jumps from its previous BGP value $X_{o}$ to $X_{1}=\frac{\rho+g+\gamma_{1}}{\rho+g+\gamma_{0}} X_{o}$ in $t_{0}$ (with $g$ indicating the new BGP value). If there is an unexpected change in one of the other exogenous parameters (including $X_{o}$ ), $g$ and $c_{R}$ immediately jump to their new BGP values and $X$ remains constant after $t_{0}$ (and only changes in $t_{0}$ if there is an exogenous change in $X_{0}$ ).

Proof. a) The demand for production labor is given by

$$
L_{D}(t)=\int_{j=0}^{N(t)}\left(\frac{b}{N(t)}\right) 1 d j+\int_{j=N(t)}^{C_{R}(t)}\left(\frac{\Omega}{N(t)}\right)(1-\beta) d j=b+\left(c_{R}-1\right)(1-\beta)
$$

The resource constraint can therefore be derived as

$$
g(t)=\frac{1}{F}\left[1-b-(1-\beta) \Omega\left(c_{R}-1\right)\right]
$$

Along a BGP, the intertemporal budget constraint of a poor household is given by

$$
\frac{N(\tau) \vartheta}{r-g}+V_{P}(\tau)=\frac{N(\tau)}{r-g}\left[m b+p_{P}(1-m)\right]
$$

The free entry condition is given by $Z=\frac{p_{P}-b}{r+\gamma}=F$, from which we get $p_{P}=b+$ $F(r+\gamma)$. Plugging this equation and equations 6 and 8 and 14 into equation 33 and solving for $g$, we obtain

$$
g=\frac{1}{F}(\vartheta-b-\rho(1-\beta) X)
$$

Plugging this equation into the resource constraint gives $c_{R}=1+\frac{1-\vartheta+\rho(1-\beta) X}{(1-\beta) \Omega}$.

This regime can only arise if poor households are rich enough to purchase one unit of each good produced with an innovative technology, which is the case if $\vartheta-b-$ $\rho(1-\beta) X>0$. IP holding firms do not find it profitable to sell exclusively to rich 
households at price $p_{R}=\Omega$ if $\frac{(\Omega-b)(1-\beta)}{r+\gamma}<F$ holds and firms selling to both income groups at price $p_{P}$ are not constrained by the presence of the traditional technology if $p_{P}<\Omega$, i.e. if $b+F(r+\gamma)<\Omega$. Plugging in the BGP value of $r=\rho+g$, both conditions are satisfied if $F(\rho+\gamma)+\vartheta-\rho(1-\beta) X<\Omega<\frac{F(\rho+\gamma)+\vartheta-b \beta}{(1-\beta)}-\rho X$ holds. $X<\tilde{X} \equiv \frac{(1-m) F}{1-\beta}>0$ again needs to hold in order to ensure that $V_{P}(t)>0$.

b) In order to show that $g$ and $c_{R}$ immediately jump to their new BGP values, it needs to be checked that along the transition path (where $V$ and $M$ adjust sluggishly) the intertemporal budget constraint of a poor household is satisfied if $g, c_{P}$ and $c_{R}$ adjust immediately and if $X$ jumps from $X_{0}$ to $X_{1}=\left[\frac{\rho+g+\gamma_{1}}{\rho+g+\gamma_{0}}\right] X_{0}$. The budget constraint of a poor household is given by

$$
\frac{N(\tau) \vartheta}{r-g}+V_{P}(\tau)=\int_{t=\tau}^{\infty}\left[b M(t)+p_{P}(N(t)-M(t))\right] e^{-r(t-\tau)} d t
$$

Plugging in equation 6 , the (forward looking) free entry condition $p_{P}=b+F\left(r+\gamma_{1}\right)$, equation 20 and $V_{P}(\tau)=N(\tau)\left[\frac{\rho+g+\gamma_{1}}{\rho+g+\gamma_{0}}\right]\left[\left(1-m_{o}\right) F-(1-\beta) X_{o}\right]$ from the proof of Proposition 2, this equation can be rewritten as

$$
g=\frac{1}{F}\left(\vartheta-b-\rho(1-\beta) X_{o}\left[\frac{\rho+g+\gamma_{1}}{\rho+g+\gamma_{0}}\right]\right)
$$

As this is the same equation as equation 34 (that determines the BGP value of $g$ ), with $X_{o}$ replaced by $X_{1}=\left[\frac{\rho+g+\gamma_{1}}{\rho+g+\gamma_{0}}\right] X_{o}$, the budget constraint of a poor household is indeed satisfied during the transition. The intertemporal budget constraint of a rich household automatically holds as $c_{R}$ is constant during the transition.

Due to a mechanism similar to that at work in Regime B, the length of IP protection has no effect on $g$ when $\gamma_{1}=\gamma_{0}$ as it leaves "total demand" unaffected and merely shifts a uniform demand across sectors and time. However, changing the length of IP protection can affect growth through valuation effects: when $\gamma_{1}<\gamma_{0}$ (for example because IP protection is prolonged), the value of initial wealth falls, leading to a reduction in inequality (as rich people hold more initial wealth) which in this regime (unlike in Regime A) leads to an increase in $g$. A reduction of $\vartheta$ or an increase in $X$ reduces $g$, as it reduces poor households' willingness to pay that drives the incentives to innovate (as, due to the restriction in pricing power, monopolists do not find it profitable to innovate in sectors the goods of which are only consumed by rich households).

\section{Regime C3}

In this regime, both rich and poor households consume all goods produced with a modern technology and also some traditionally produced goods $\left(C_{R}(t)>C_{p}(t)>N(t)\right)$. Therefore, all IP protected goods are sold at the price $p=z_{R}=z_{P}=\Omega$. 
Proposition 10. a) A BGP in Regime C3 exists if $\frac{\Omega-b}{F}-\rho-\gamma>0, \vartheta+F(\rho+\gamma)-$ $\rho X(1-\beta)>\Omega$ and if $X<\tilde{X}>0$. Along a $B G P, g=\frac{\Omega-b}{F}-\rho-\gamma, c_{P}=\frac{\vartheta+F(\rho+\gamma)-\rho X(1-\beta)}{\Omega}$ and $c_{R}=\frac{\frac{1-\beta \vartheta}{1-\beta}+F(\rho+\gamma)+\rho X \beta}{\Omega}$.

b) $g, c_{P}$ and $c_{R}$ immediately jump to their new BGP values when there is a change in $\gamma=\gamma_{1}=\gamma_{0}$ or in another exogenous parameter (including X). For any initial level of $\gamma$ compatible with the existence of a BGP in Regime C3, both rich and poor households benefit from an increase in the length of IP protection $T$ (i.e. from a reduction in $\gamma$ ).

Proof. a) The resource constraint is given by

$$
g=\frac{1}{F}\left[1-b-(1-\beta) \Omega\left(c_{R}-1\right)-\beta \Omega\left(c_{P}-1\right)\right]
$$

The intertemporal budget constraints of poor and a rich household are given by

$$
\begin{gathered}
\frac{N(\tau) \vartheta}{r-g}+V_{P}(\tau)=\frac{N(\tau)}{r-g}\left[m b+\left(c_{P}-m\right) \Omega\right] \\
\frac{N(\tau) \frac{1-\beta \vartheta}{1-\beta}}{r-g}+V_{P}(\tau)+X N(\tau)=\frac{N(\tau)}{r-g}\left[m b+\left(c_{R}-m\right) \Omega\right]
\end{gathered}
$$

Combining these equations with the free entry condition $\frac{\Omega-b}{r+\gamma}=F$ and the Euler equation $r=\rho+g$ allows the derivation of the BGP values of $g, c_{P}$ and $c_{R} . X<\tilde{X} \equiv \frac{(1-m) F}{1-\beta}>0$ again needs to hold in order to ensure that $V_{P}(t)>0$.

b) Proceeding as in the previous proofs, it can be shown that both intertemporal budget constraints are satisfied during the transition phase if $X$ is constant and if $g, c_{P}$, and $c_{R}$ immediately jump to their new BGP values while $m$ and $V$ adjust sluggishly. This again implies that there are no transitional dynamics in the variables $g, c_{P}$, and $c_{R}$. As in Regime $\mathrm{C} 1$, a reduction in $\gamma=\gamma_{1}=\gamma_{0}$ again reduces $c_{P}$ and $c_{R}$ by the same absolute amount. As intertemporal utility $\left(U_{i}(\tau)=\frac{\ln N(\tau)}{\rho}+\frac{\ln c_{i}}{\rho}+\frac{g}{\rho^{2}}\right)$ is concave in $c_{i}$ and linear in $g$, rich households therefore always benefit from a reduction in $\gamma$ if poor households benefit from it, i.e. if $\frac{\partial U_{P}}{\partial \gamma}<0$ holds. The latter inequality can be shown to hold if $\vartheta-\rho X(1-\beta)+F \gamma>0$, which is always satisfied if $c_{P}>1$ holds. Therefore, both income groups always benefit from a reduction in $\gamma$ in this regime.

In this regime, increasing $T$ increases $g$ and, as in Regime $\mathrm{C} 1$, reduces $c_{P}$ and $c_{R}$ by the same absolute amount. Inequality has no effect on growth as the market size for innovative goods is always equal to one and as prices are always given by $\Omega$.

\section{Shifts between regimes}

While the above sections analyzed the effects that IP policies and inequality have on growth within a given regime, a change in IP protection (or in inequality) can also 
lead to a switch between regimes: Suppose that $\vartheta-b-\rho(1-\beta) X>0$ and $A \equiv$ $\frac{\left(1-\frac{1-\vartheta}{(1-\beta) \Omega}-\frac{X \rho}{\Omega}\right)(b \beta+\Omega(1-\beta))-\vartheta-F \rho+(1-\beta) X \rho}{F}>0$ hold. The first condition implies that poor households can always afford to purchase all goods on which IP protection has expired (i.e. $C_{P}>M$ (Condition A) always holds), while the second condition implies that $\Omega$ is sufficiently large so that the economy is in Regime A when $\gamma=0$ (infinite IP protection). We can then infer from Propositions 7, 9 and 10 that the economy is in Regime A if $0 \leq \gamma \leq A$, in Regime C1 if $A<\gamma<B \equiv \frac{b \beta+\Omega(1-\beta)-\vartheta-F \rho+(1-\beta) X \rho}{F}$, in Regime C2 if $B \leq \gamma \leq C \equiv \frac{\Omega-F \rho-\vartheta+(1-\beta) X \rho}{F}$ and in Regime C3 if $C<\gamma<\frac{\Omega-b}{F}-\rho$.

Starting from infinite IP protection, a reduction in the length of IP protection $T$ (i.e. an increase in $\gamma$ ) leads to a shift to Regime $\mathrm{C} 1$, as it pushes firms that sell exclusively to rich households to increase their prices up to the maximal level $\Omega$. A further reduction in $T$ then makes it unprofitable to sell exclusively to rich households and leads to a switch to Regime C2. If $T$ is reduced even further, firms have to raise prices of all IP protected goods to $\Omega$ and there is a switch to Regime C3. As the effect that inequality has on growth is different in the different regimes, changing the length of IP protection can therefore change the effect that inequality has on growth: While inequality is good for growth when $T$ is large (Regime A), its effect on growth becomes negative when $T$ decreases (Regimes $\mathrm{C} 1$ and $\mathrm{C} 2$ ) and it ceases to affect growth when $T$ is reduced even further (Regime C3).

If parameters are such that the economy is initially in Regime A (e.g. $\Omega$ is large enough and $\gamma$ small enough), it can, moreover, be shown that a continuous reduction in patent breadth $\bar{p}$ also first leads to a switch to Regime $\mathrm{C} 1$, then to Regime $\mathrm{C} 2$, and finally to Regime C3. Therefore, a reduction in patent breadth also makes it less likely that inequality is good for growth.

\section{B8: A simple static model}

In the following, it is shown that some of the main results of the analysis also hold in simple static version of the model in which there are no $R \& D$ spillovers and in which incomes and production costs are exogenously given. Suppose that there are only two periods: in the first, firms decide how much to invest in $\mathrm{R} \& \mathrm{D}$ and in the second, goods are produced and consumed. Utility is now given by $U_{i}=\int_{j=0}^{\infty} c_{i}(j) d j$ with $c_{i}(j) \in\{0,1\}$. Incomes of rich (poor) households are given by $Y_{R}\left(Y_{P}\right)$ and the fraction $\beta$ of the households are poor. Inventing a good costs $F$ and producing one unit of an invented good costs $b^{68}$. There is again free entry into $R \& D$. Innovations are protected by intellectual property rights, that are, however, only enforced with probability $\Delta$. In the case of non-enforcement,

\footnotetext{
${ }^{68}$ Incomes can again be thought of as labor endowments and costs as labor input requirements (assuming that the wage rate for one unit of labor is normalized to one).
} 
there is marginal cost pricing. When the measure $N$ or goods are invented, there are therefore $\Delta N$ goods on which IPRs are enforced and $M \equiv(1-\Delta) N$ goods which are competitively supplied. Rich (poor) households consume the measure $C_{R}\left(C_{P}\right)$ of the goods.

As in the main model, $C_{R}=N$ holds in equilibrium and two regimes can arise: when $C_{P}>M$, demand for IP protected goods is heterogeneous, while it is homogenous when $C_{P}<M$ holds.

Proposition 11. a): When demand is heterogeneous, increasing the strength of IP protection $\Delta$ increases innovation $N$ and reduces the consumption share $c_{P} \equiv \frac{C_{P}}{N}$ of poor households. While rich households always prefer full IP protection $(\Delta=1)$, poor households prefer an interior strength of protection $\Delta<1$ for certain parameter constellations.

b): When demand is homogeneous, innovation $N$ is independent of the strength of IP protection $\Delta$.

Proof. a): Suppose that innovators charge price $p_{R}$ when they sell exclusively to rich households and $p_{P}$ when they sell to both rich and poor households. Free entry then implies that $\pi_{P}=\Delta\left(p_{P}-b\right)=F$ and $\pi_{R}=\Delta(1-\beta)\left(p_{R}-b\right)=F$ must hold, from which we obtain the prices as:

$$
\begin{gathered}
p_{P}=\frac{F}{\Delta}+b \\
p_{R}=\frac{F}{\Delta(1-\beta)}+b
\end{gathered}
$$

The budget constraints of rich and poor households are given by

$$
\begin{gathered}
Y_{R}=b M+p_{P}\left(C_{P}-M\right)+p_{R}\left(C_{P}-C_{P}\right) \\
Y_{P}=b M+p_{P}\left(C_{P}-M\right)
\end{gathered}
$$

Combining the above four equations and taking into account that $M=(1-\Delta) N$ allows to derive

$$
\begin{gathered}
N=C_{R}=\frac{Y_{R}(1-\beta)(F+b \Delta)+F \beta Y_{P}}{F b+F^{2}+\Delta(1-\beta)\left(F b+b^{2}\right)} \\
C_{P}=\frac{1}{(F+b \Delta)}\left[Y_{P} \Delta+F(1-\Delta) C_{R}\right]
\end{gathered}
$$

Deriving with respect to $\Delta$ gives $\operatorname{sign}\left(\frac{\partial N}{\partial \Delta}\right)=\operatorname{sign}\left[\left(Y_{R}-Y_{P}\right) \beta\left(F^{2}+b F\right)\right]>0$, implying that innovation increases in $\Delta$ and that rich households prefer $\Delta=1$ as $C_{R}=N$. As $\frac{\partial C_{P}}{\partial C_{R}}=\frac{F(1-\Delta)}{F+b \Delta}<1, c_{P}=\frac{C_{P}}{C_{R}}$ falls when $\Delta$ increases (as the latter increases $C_{R}$ ). Moreover, it can be shown that sign $\left.\frac{\partial C_{P}}{\partial \Delta}\right|_{\Delta=1}<0$ holds for certain parameter constellations, for example when $b=1$ and $F>1$, or when $b$ is sufficiently large and $F$ is sufficiently small. In these cases, poor households therefore benefit from reducing IP protection below the maximal level. 
b): As above, free entry implies

$$
p_{R}=\frac{F}{\Delta(1-\beta)}+b
$$

The budget constraints are given by

$$
\begin{gathered}
Y_{R}=b M+p_{R}\left(C_{R}-M\right) \\
Y_{P}=b C_{P}
\end{gathered}
$$

Combing the equations and taking into account that $M=(1-\Delta) N$ gives:

$$
N=C_{R}=\frac{Y_{R}}{b+\frac{F}{1-\beta}}
$$

As this expression is independent of $\Delta$, innovation does not depend on the strength of IP protection in this case (as long as $\Delta$ is sufficiently small to guarantee that the economy is indeed in the regime in which $C_{P}<M$ holds).

As there are no spillovers, the consumption and utility of a household who does not consume any IP protected goods $\left(C_{i}<M\right)$ is simply given by $C_{i}=U_{i}=\frac{Y_{i}}{b}$ in this setting and is therefore independent of $\Delta$. 